\title{
Influence of Hydrogen Atoms on the Growth of carbon based Nanoparticles and of Thin Films from reactive Plasmas
}

\section{Dissertation}

\author{
Zur \\ Erlangung des Grades \\ eines Doktors der Naturwissenschaften \\ in der Fakultät für Physik und Astronomie \\ der Ruhr Universität Bochum
}

Von

Olivera Stepanović

aus Niš, Serbien

Bochum, 2006 
Gedruckt mit der Genehmigung der

Fakultät für Physik und Astronomie der Ruhr-Universität Bochum

Referent: Prof. Dr. Jörg Winter

Koreferent: Prof. Dr. Henning Soltwisch

Tage der mündlichen Prüfung: 04. 12. 2006 
To my family 


\section{Contents}

1 Introduction 1

2 Experiment $\mathbf{5}$

2.1 The MW/UHF double plasma experimental setup . . . . . . . . . 6

2.2 The RF ICP/UHF double plasma experimental setup . . . . . . . . 8

2.3 The UHF plasma as source for hydrogen atoms . . . . . . . . . . . . 14

2.4 Sample preparation and analysis . . . . . . . . . . . . . . . 17

3 Diagnostics $\quad 19$

3.1 Ellipsometry . . . . . . . . . . . . . . . . . . . 19

3.1.1 Light polarization . . . . . . . . . . . . . . . . 19

3.1.2 Basic principles of the ellipsometry measurements . . . . . . . 19

3.1.3 Ellipsometer configuration . . . . . . . . . . . . . . . 22

3.1 .4 Optical constants . . . . . . . . . . . . . . 22

3.2 Infrared and Raman spectroscopy . . . . . . . . . . . . . . . . . . . 24

3.2 .1 Infrared spectroscopy . . . . . . . . . . . . . . . 26

3.2 .2 Raman spectroscopy . . . . . . . . . . . . . . 30

3.2 .3 Spectra manipulation . . . . . . . . . . . . . . 33

3.3 Mass spectrometry . . . . . . . . . . . . . . . . . . 35

4 The role of hydrogen atoms in dust particle formation 37

4.1 Introduction . . . . . . . . . . . . . . . . . . . 37

4.2 Some aspects of dust particle formation . . . . . . . . . . . . . 39

4.2.1 Time development of particle formation and growth . . . . . . 39

4.2 .2 Charging of particles in plasmas . . . . . . . . . . . . . 40

4.2.3 Forces acting on particles in plasmas . . . . . . . . . . . . . 42

4.3 Results . . . . . . . . . . . . . . . . . . . . . . 46

4.3.1 Influence of hydrogen atoms on dust particle formation . . . . 47

4.3.2 Mass spectra and hydrogen dilution . . . . . . . . . . . . . . . 49

4.3.3 Conclusions . . . . . . . . . . . . . . . . . . . . . 61 
5 The role of hydrogen atoms in a-C:H film formation 63

5.1 Introduction . . . . . . . . . . . . . . . . . . 63

5.2 Film growth . . . . . . . . . . . . . . . . . . 65

5.2.1 Influence of hydrogen atoms on the deposition speed . . . . 65

5.2 .2 Role of the precursor gas . . . . . . . . . . . . . . . . 67

5.2 .3 Influence of hydrogen atoms on the etching speed . . . . . . . 68

5.2 .4 Dependence on ion energy . . . . . . . . . . . 70

5.3 Film properties . . . . . . . . . . . . . . . . . . 72

5.3.1 Influence of hydrogen atoms on the optical constants . . . . . 72

5.3.2 Influence of hydrogen atoms on the film structure . . . . . . . 74

5.4 Thin film deposition in dusty plasmas . . . . . . . . . . . . . . 77

5.4.1 Film growth . . . . . . . . . . . . . . . 77

5.4.2 Film properties and film structure . . . . . . . . . . . 81

5.4 .3 Conclusions . . . . . . . . . . . . . . . . . 83

6 Summary and Conclusions $\quad 84$ 


\section{Chapter 1}

\section{Introduction}

In 1971 Aisenberg and Chabot deposited for the first time hydrocarbon films [1]. They used an ion-beam deposition technique. These films had some material and physical properties similar to diamond and therefore they got the name diamondlike carbon films (DLC). Few years later, in 1976, Holland and Ojha [2] used an RF plasma enhanced chemical vapour deposition technique (PECVD) to produce films containing some amount of hydrogen. These films also showed very interesting properties and since then amorphous carbon films attracted a great attention worldwide. Besides these two major allotropic modifications, it was found in 1980s that carbon is able to build up one more - the buckminster fullerene $\mathrm{C}_{60}$ form. The interest for nanotubes, as a subset of fullerenes, in academic and industrial sphere grows every day.

For the deposition of hydrocarbon films different techniques, reactor geometries and gases are used. One of the most exploited techniques in plasma processing is the plasma enhanced chemical vapour deposition. Plasma technology became irreplaceable today in the industry of integrated circuits and microchips, surface processing, selective etching or surface treatment. The reactor consists of two parallel electrodes. The RF power is coupled to one of them while another is grounded. Usually the substrate is mounted on the powered electrode to achieve harder coatings. The applied RF power produces a plasma between electrodes, a complex system of electrons, positive and negative ions, neutrals and photons. The properties of the hydrocarbon films can be adjusted and optimized over a wide range of deposition conditions.

Nowadays carbon based materials are widely used in many technological applications thanks to the variety of properties they possess. This versatility comes from the fact that they can, on one side, have high mechanical hardness, low friction coefficient, high wear resistance, and be optically transparent in the visible and infrared range, chemically inert to both acids and bases. On the other hand, they can be very soft and opaque, easy for scratching. Where does this heterogeneity in carbon materials properties come from? 


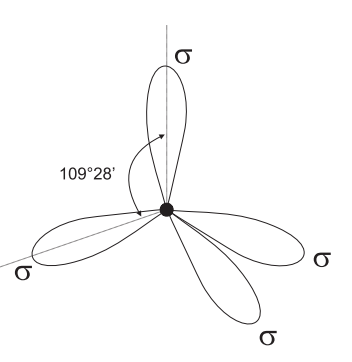

$\mathrm{sp}^{3}$

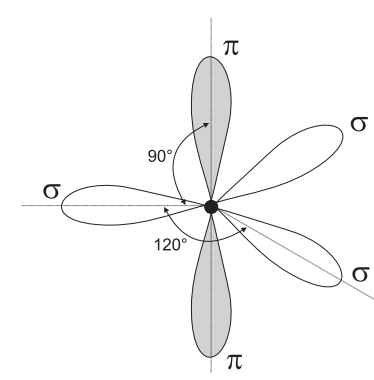

$\mathrm{sp}^{2}$

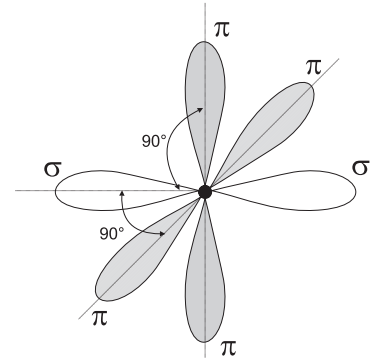

$\mathrm{sp}$

Figure 1.1: Schematic representation of tree possible hybridization states in which carbon atom can exist.

Materials owe this heterogeneous properties to the fact that carbon atoms can form different structures - from crystalline to disordered - because it can exist in three different hybridization states: $s p^{3}, s p^{2}$ and $s p^{1}$ as it is shown in Figure 1.1. In the $s p^{3}$ configuration four carbon valence electrons are settled in the corners of the regular tetrahedron (the carbon atom is in the center of that tetrahedron). Diamond has such a configuration. In this configuration carbon makes strong $\sigma$ bonds with neighboring carbon atoms overlapping the orbitals in the end-on mode building a very strong network. The angle between bonds is $109^{\circ} 28^{\prime}$ and this value is found only in the molecules with the so-called tetrahedral symmetry.

In the $s p^{2}$ configuration three of four carbon atoms lie in the same plane building strong $\sigma$ bonds while the fourth electron is in the $\pi$ orbital which lies in the plane normal to the $\sigma$ bonding plane. These $\pi$ orbitals form a $\pi$ bond which is weaker in comparison with the $\sigma$ bond and the mode of overlapping in this case is sideway on. The angle between the $\sigma$ bonds is $120^{\circ}$. These $\pi$ electrons can be delocalized as in the olefinic systems or localized as in benzene $\left(\mathrm{C}_{6} \mathrm{H}_{6}\right)$ or graphite.

In the linear or $s p^{1}$ configuration two electrons lie along one axis and form $\sigma$ bonds at angles of $180^{\circ}$ whereas the other two electrons lie in the $\pi$ orbitals forming an angle of $90^{\circ}$ to each other and to the $s$ orbitals. This $s p^{1}$ acetylene-like chain form is very rare in the amorphous carbon films.

Going from $s p^{3}$ to $s p^{2}$ and $s p^{1}$ configuration the electron density increases near the carbon nucleus and the carbon-carbon bond length shortens and becomes stronger. The average value of the bond length in $s p^{3}$ configuration is $0.154 \mathrm{~nm}$, in $s p^{2} 0.133$ $\mathrm{nm}$ and in $s p^{1} 0.120 \mathrm{~nm}$.

The influence of hybridization on the material properties is nicely illustrated by the big difference between diamond and graphite properties. While diamond, with $100 \% s p^{3}$ bonded carbon atoms, is the hardest material on earth, transparent, a very 


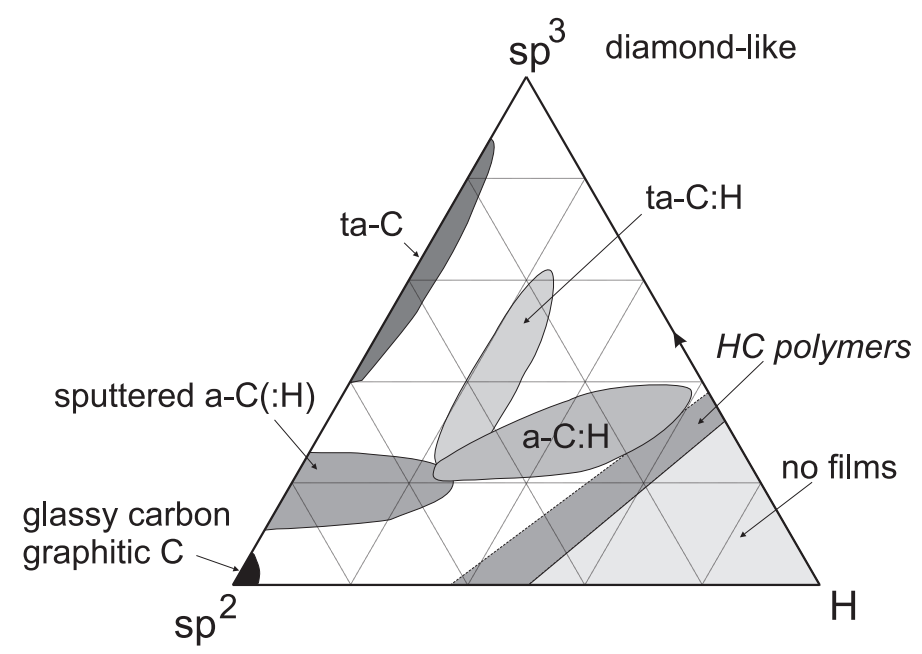

Figure 1.2: Ternary phase diagram of amorphous hydrocarbons. The tree corners correspond to diamond, $s p^{3}$, graphite, $s p^{2}$, and $100 \%$ hydrogen content (shadowed area in the lower right corner where it is not possible to deposit any film). Amorphous hydrogenated films, a: $\mathrm{C}-\mathrm{H}$, are settled in the limited region in this diagram (4).

good insulator having an energy band gap of $5.5 \mathrm{eV}$, graphite, consisting only of $s p^{2}$ hybridized carbon atoms, is very soft, black, opaque material with $0 \mathrm{eV}$ energy band gap. Between these two extremes amorphous hydrogenated films are located.

Hydrogen is an obligatory constituent in the amorphous carbon films deposited from hydrocarbon gases in PECVD. Depending on the growth technique, the growth conditions and the used hydrocarbon precursor gas, a group of so-called amorphous hydrogenated carbon films with different structure and content of hydrogen in both forms - molecular and atomic can be grown. The fact that hydrogen can be incorporated into the film during the growth process is a further complication because the film structure and the film properties are also influenced.

The composition of the hydrocarbon films can be presented as a function of $s p^{3}$, $s p^{2}$ and hydrogen content in the form of a ternary phase diagram (see Fig. 1.2) as first was done by Smith [3] and later used by Jacob and Möller [4]. In the $s p^{3}$ corner are diamond-like films with a significant fraction of $s p^{3}$ bonds. In the lower left corner $\left(s p^{2}\right)$ are films with graphitic structure ranging from graphite to glassy carbon. The two shaded areas in the lower right corner $(\mathrm{H})$ correspond to the films rich in hydrogen content without stable structure and to the conditions where it is not possible to deposit film because volatile hydrocarbon products are produced due to the very high hydrogen concentration. Between these extreme groups amorphous hydrogenated films are placed around the center of the ternary phase diagram. These films consist of a mixture of all these three forms of hybridized 
carbon atoms in different ratios.

Besides the polymerization process occurring on the surface leading to the film deposition, polymerization process in reactive gas plasmas can spontaneously occur also in the plasma volume leading to dust particle formation. This process is a very well known phenomenon since 1920 when Langmuir and co-workers observed and described it for the first time [5]. Dusty particles attract for years great attention in the industry. The particles change the plasma properties, and can be embedded in the film matrix causing the malfunctioning of the deposited layer. This is the reason why they are recognized as killer particles in the microelectronic industry.

On the other hand, in addition to these technological aspects, dusty particles, the formation process and their structure attract enormous attention of astrophysicists because carbonaceous dusty particles are found in interstellar medium, they participate in the formation of stars and solar systems etc.

The aim of this work is to study the role hydrogen atoms play in the amorphous hydrogenated films structure and the surface modification as well as in the carbonaceous dust particle formation. For these investigations a specially designed experiment is developed. It consists of two coupled plasmas - a radiofrequency (RF) and an ultra high frequency (UHF) plasma. RF plasma dissociates the precursor gas molecules creating active species while the second, UHF plasma, operating with molecular hydrogen, acts as an independent and controllable source of hydrogen atoms.

This work is organized on the following way. Chapter 2 presents the experiment, first the double plasma experimental setups consisting of a surface wave sustained discharge (SWSD) in one case and an inductively coupled plasma (ICP) in the other and an ultra high frequency (UHF) discharge. The techniques used in the thin film analysis and characterization are reviewed in chapter 3. Chapter 4 contains the results about the role of hydrogen atoms during the nanoparticle formation, particularly about the suppression of dust particle formation in the presence of an additional flux of hydrogen atoms. Chapter 5 deals with the interaction of hydrogen atoms and the surface of growing film and their influence on the growth/etching speed of the film. Finally, chapter 6 contains the general summary and conclusions of this work. 


\section{Chapter 2}

\section{Experiment}

In every hydrocarbon discharge hydrogen molecules and atoms are created inherently due to the dissociation of precursor molecules. Molecules and atoms stay in the discharge long enough to play an important role in the surface processes and the plasma chemistry and to contribute significantly to the flux of radicals impinging onto the surface. In order to be able to study the role hydrogen atoms play in the thin film growth, and consequently the structure, and the plasma chemistry during the dust particles formation, an independent source of hydrogen atoms is needed. The flowing afterglow of the second discharge is then used to enhance artificially and in a controllable way the flux of added hydrogen atoms.

During this work two different setups of double plasma experiments are used. In the first experimental setup a microwave (MW) expanding plasma at $2.45 \mathrm{GHz}$ is combined with an ultra high frequency (UHF) plasma. The expanding plasma is used for the creation of active species, while the UHF plasma is used for the creation of hydrogen atoms through the dissociation process of hydrogen molecules in the discharge. This experimental double-plasma setup is used for thin film deposition and etching and is described together with the basic principles of the MW discharge operation in section 2.1 .

The second experimental setup consists of a radio frequency (RF) plasma at standard $13.56 \mathrm{MHz}$ in combination with the UHF plasma used in the first experimental setup. In this setup the RF discharge is used for the creation of active species and the UHF plasma is again used for the creation of hydrogen atoms from hydrogen molecules. The second double-plasma setup is used for thin film processing and for the investigation of dust particle formation. The description of this setup and the experimental conditions are given in detail in section 2.2 .

An UHF plasma at 144.1 MHz is employed as an additional source of hydrogen atoms. They are injected, independent of the precursor gas, into the hydrocarbon plasma or directed toward the treated surface of the sample. This plasma is described in section 2.3 . 
The samples used in the deposition and the etching experiments together with the sample preparation technique are described in section 2.4.

\subsection{The MW/UHF double plasma experimental setup}

Fig. 2.1 presents a sketch of the MW/UHF double plasma experimental setup. It mainly consists of a high vacuum chamber, a microwave plasma, an UHF plasma and a sample holder. The chamber has a cylindrical shape with a height $590 \mathrm{~mm}$ and a diameter of $340 \mathrm{~mm}$. The microwave plasma is ignited via the surfatron 2 while the hydrogen plasma is ignited via the surfatron 1 . The mass spectrometer (MS) is used for the monitoring of the plasma composition.

The expanding plasma of a traveling surface wave, first described in 1970s [6], is excited by means of a waveguide surfatron at $2.45 \mathrm{GHz}$. It is used for the production of active species in the precursor gas for surface processing. During these experiments only the argon discharge is used as expanding plasma while as precursor gases methane and acetylene are used. The precursor gases are let in from the top of the chamber.

The chamber is pumped by a $1500 \mathrm{l} / \mathrm{s}$ turbo molecular pump (Pfeiffer TMU 1601) down to a base pressure of $10^{-6}$ mbar. During operation the pressure in the chamber filled with gas is of the order of $10^{-3}$ mbar, being measured by a second Baratron $627 \mathrm{~A}$ (pressure range $10^{-1}-10^{-5} \mathrm{mbar}$ ). The pump is directly mounted on the chamber through a $250 \mathrm{~mm}$ port to achieve the maximum possible conductance.

The launcher is supplied with a power of up to $1 \mathrm{~kW}$ from a Thompson-CSF TH 2404 klystron working at $2.45 \mathrm{GHz}$ which is driven by a Marconi signal source type 6056B microwave generator. The transmitted and the reflected power are measured with HP 436A powermeters which are damped by two directional couplers (damping $40 \mathrm{~dB}$ ). There are variants of surface wave launchers. When they have a coaxial form they are termed as surfatron [7] while the launchers using a rectangular waveguide are called surfaguide [8]. The launcher is easy to operate and can work over a wide frequency range. Plasmas produced on this way have low fluctuations in electron density and are very stable. Fig. 2.2 presents a cross-section of the surfatron. It consist of two coaxial cylinders and is terminated at one end with a circular gap. The power is coupled to a coupler (not presented in figure) movable in the radial direction by a coaxial cable. The sliding contacts ensure the electrical connection between the coupler and the waveguide wall. The plungers tune the impedance 


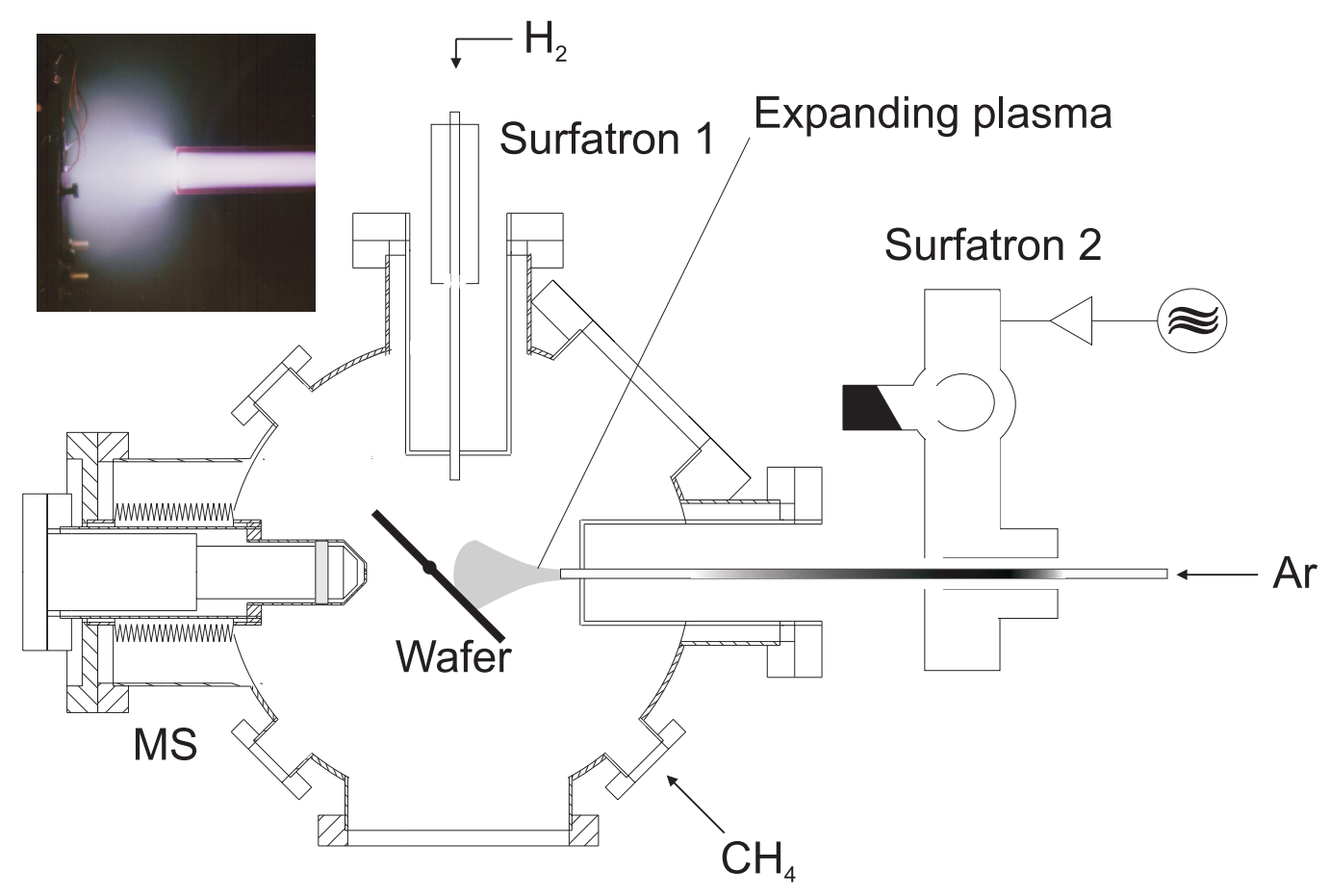

Figure 2.1: Top view sketch of the MW/UHF double plasma experimental setup. Two plasma sources are used for the creation of $\mathrm{Ar}^{+}$and hydrogen atoms from $\mathrm{H}_{2}$ via the surfatron 1 and 2, respectively, and the position of the sample. The photo in the left upper corner presents the expansion ball formed at the end of the quartz tube after the plasma expands.

maximizing the electrical filed at gap. The UHF electric field extends through the gap and excites the azimuthally symmetric surface wave sustaining the plasma in the dielectric tube.

Argon plasma is produced in a quartz tube with an inner diameter of $10 \mathrm{~mm}$, an outer of $12 \mathrm{~mm}$ and a length of $95 \mathrm{~cm}$. Around the tube a cylindrical metal grid with the diameter of $5 \mathrm{~cm}$ is placed over the whole length to prevent microwave radiation. The microwave propagates through a three dielectrics cylindrical geometry (plasma, quartz, air) and using the fact that the surface wave is an eigenmode of a bounded plasma [9] the microwave generates its own guiding structure. The gas flow through the tube is regulated by mass flow controllers (MKS flowmeters) with maximum flows of 10, 50 and $100 \mathrm{sccm}$ (standard cubic centimeter per minute). The upstream pressure is measured by a capacitance manometer Baratron $626 \mathrm{~A}$ (pressure range $0.1-1$ mbar). Due to the strong heating during the plasma operation the system is air-cooled. The length of the plasma depends on the applied power, the frequency of operation and the gas pressure. Because of the high pressure gradient between the tube (1 mbar) and the chamber $\left(10^{-3} \mathrm{mbar}\right)$, at the end of the tube the plasma 


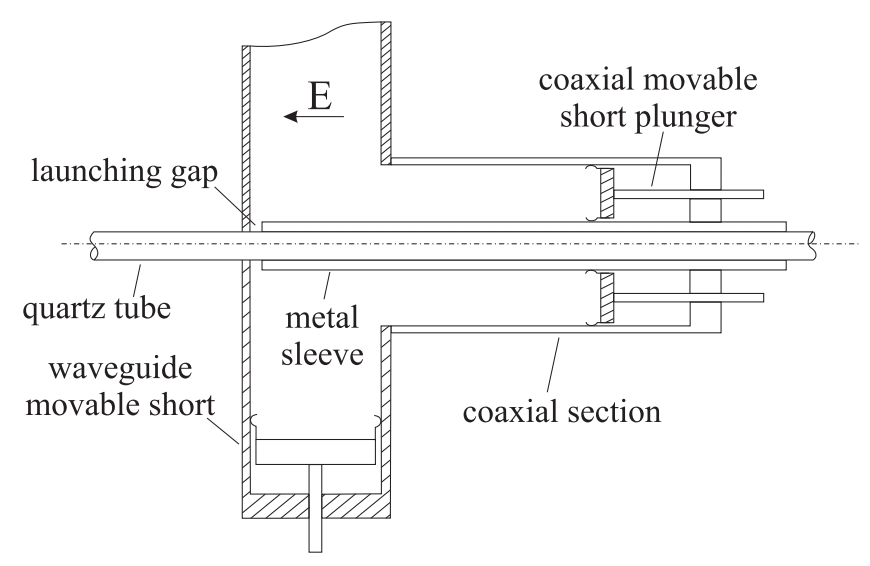

Figure 2.2: Sketch of the axial cross-section of the waveguide-surfatron.

expands in form of a ball for power values above $300 \mathrm{~W}$. In the region of expansion the plasma is not bounded by the wall of the quartz tube so wall recombination and wall loss processes do not exist in this region. The photo of the so-called plasma ball is presented in the Fig. 2.1 in the left upper corner. The expanding plasma ball is the region where reactive species (radicals and ions) are created in the interaction between the precursor molecules and the argon ions. This species are then deposited onto the silicon wafer, which is placed in a sample holder in front of the plasma ball, under an angle of $45^{\circ}$.

The left graph in Fig. 2.3 presents the intensity ratio of the measured lines in the mixture of He at $677.8 \mathrm{~nm}$ and $\mathrm{Ar}$ at $677.7 \mathrm{~nm}$ at different positions along the discharge starting from the surfatron $(z=-37 \mathrm{~cm})$ to the expansion region $(z=$ $0 \mathrm{~cm}$ ) for three different gas flows (He : $\mathrm{Ar}=7.5: 7.5,30: 30$ and $60: 60 \mathrm{sccm}$ ) [10]. After applying the corona model, described in [11], the variation of the electron temperature along the discharge can be derived from the intensity ratios of these lines. The result is presented in the right graph in Fig. 2.3. The intensity ratio, and consequently the electron temperature, sharply increases when approaching the end of the tube. The expanding plasma is studied in details in [12].

The UHF plasma (surfatron 1) for the production of hydrogen atoms is described in detail in section 2.3 .

\subsection{The RF ICP/UHF double plasma experimental setup}

Fig. 2.4 presents a sketch of the RF/UHF double plasma experimental setup where the inductively coupled plasma is combined with the UHF plasma. This setup 

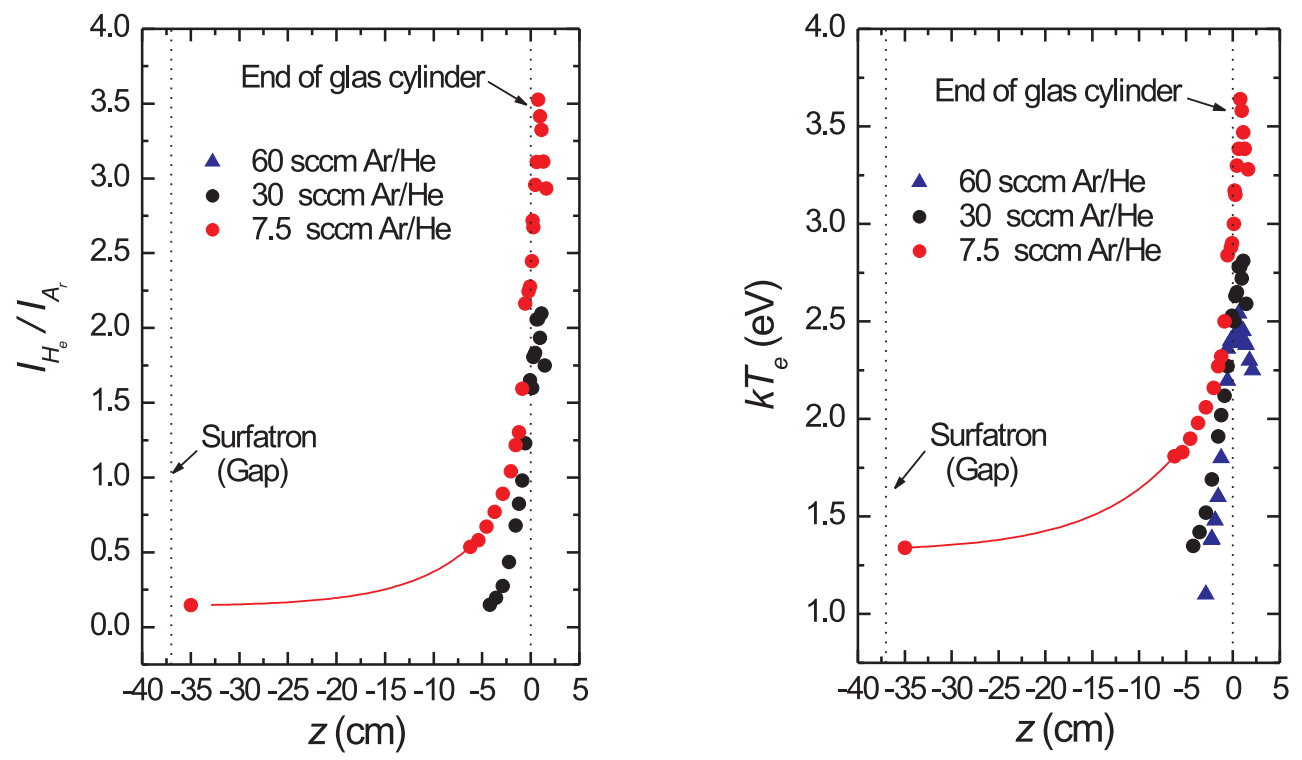

Figure 2.3: The left graph shows the ratio of the helium line intensity at $\lambda=677.8 \mathrm{~nm}$ to the argon line intensity at $\lambda=677.7 \mathrm{~nm}$ in mixtures of both gases (He and Ar have equal flows as indicated in both graphs) for different axial positions. The right graph shows the calculated values of the electron temperature along the discharge [10].

consists of a reactor of cylindrical shape and a glass cylinder which is connected through one O-ring sealing with the main reaction chamber. The inner diameter of the reactor is $60 \mathrm{~cm}$ and the height $34 \mathrm{~cm}$. From the RF generator, coupled through a matching network, the power is supplied to the antenna which is placed on the air side of the quartz cylinder and has no direct contact to the plasma. In this setup the antenna is a five-turn planar coil (pancake configuration). The inner end of the coil is powered while the outer end is grounded. The driving frequency applied to the coil is $13.56 \mathrm{MHz}$. The lower electrode is grounded and permanently water cooled ensuring a constant temperature during the processing. The distance between the electrodes is $8 \mathrm{~cm}$.

The discharges run at gas pressures of 0.05 to 2 mbar. The typical electron density in an inductively coupled discharge is $n_{e}=10^{10}-10^{12} \mathrm{~cm}^{3}[13,14]$. During the present experiments methane and acetylene are used as precursor gases with injection of molecular/atomic hydrogen through the glass cylinder. The reaction chamber is evacuated by a roots pump (Pfeiffer WKP 250) and a Pfeiffer rotary vane pump for rough vacuum down to about $10^{-3}$ mbar and a turbomolecular pump (Pfeiffer TMU $400 \mathrm{MC} / \mathrm{T}$ ) to reach a high vacuum pressure of $10^{-6}$ mbar. The precursor 

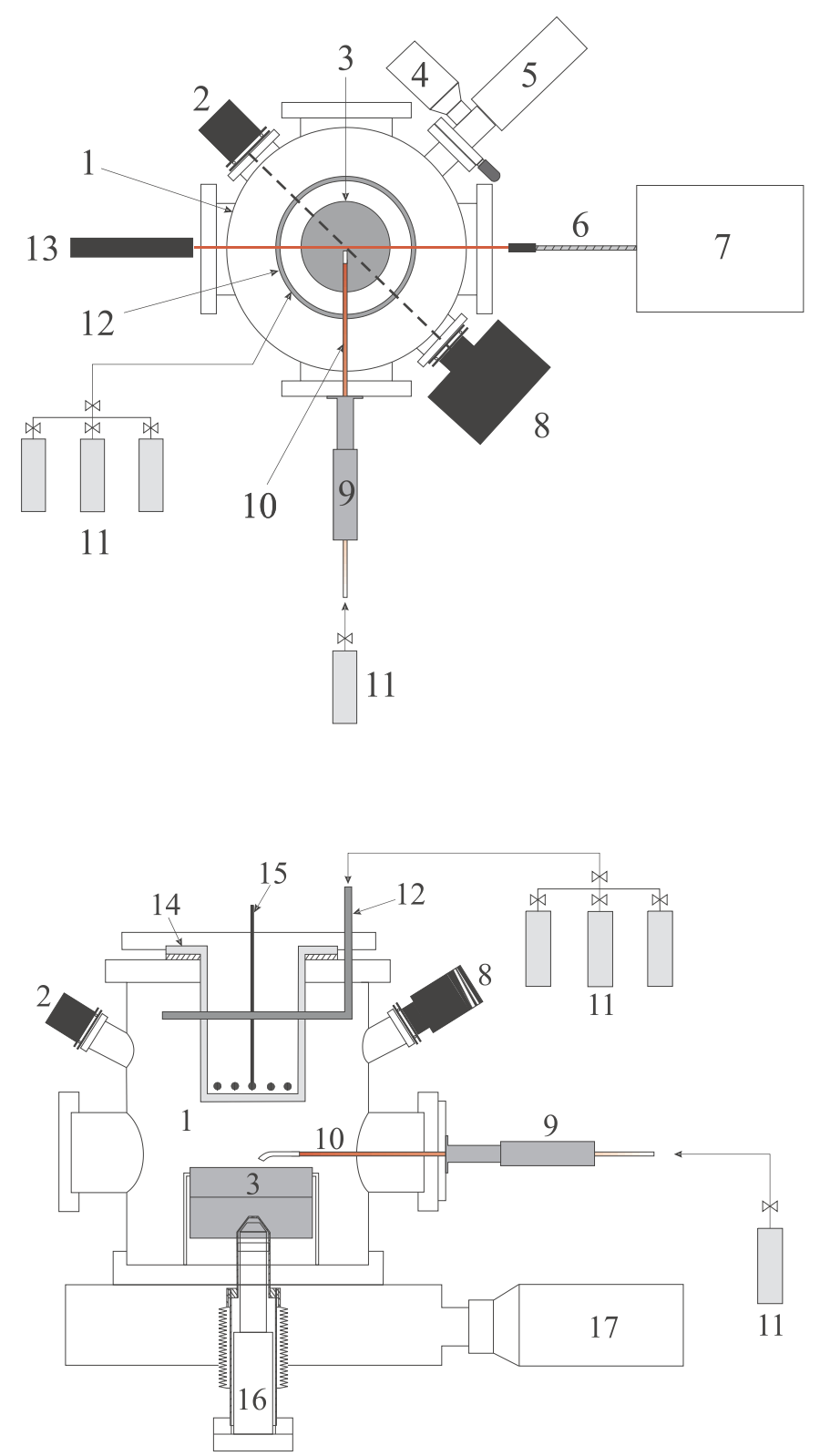

Figure 2.4: Sketch of the RF IC/UHF double plasma experimental setup. Up: top view, down: side view. 1. chamber, 2. ellipsometer (polarizer), 3. (lower) electrode, 4. pump for mass spectrometer, 5. mass spectrometer (rest gas analyzer), 6. optical cable, 7. optical spectrometer, 8. ellipsometer (analyzer), 9. surfatron, 10. quartz tube, 11. gas, 12. O-ring, 13. He-Ne laser, 14. quartz cylinder, 15. coil, 16. mass spectrometer (plasma process monitor), 17. turbomolecular pump. 

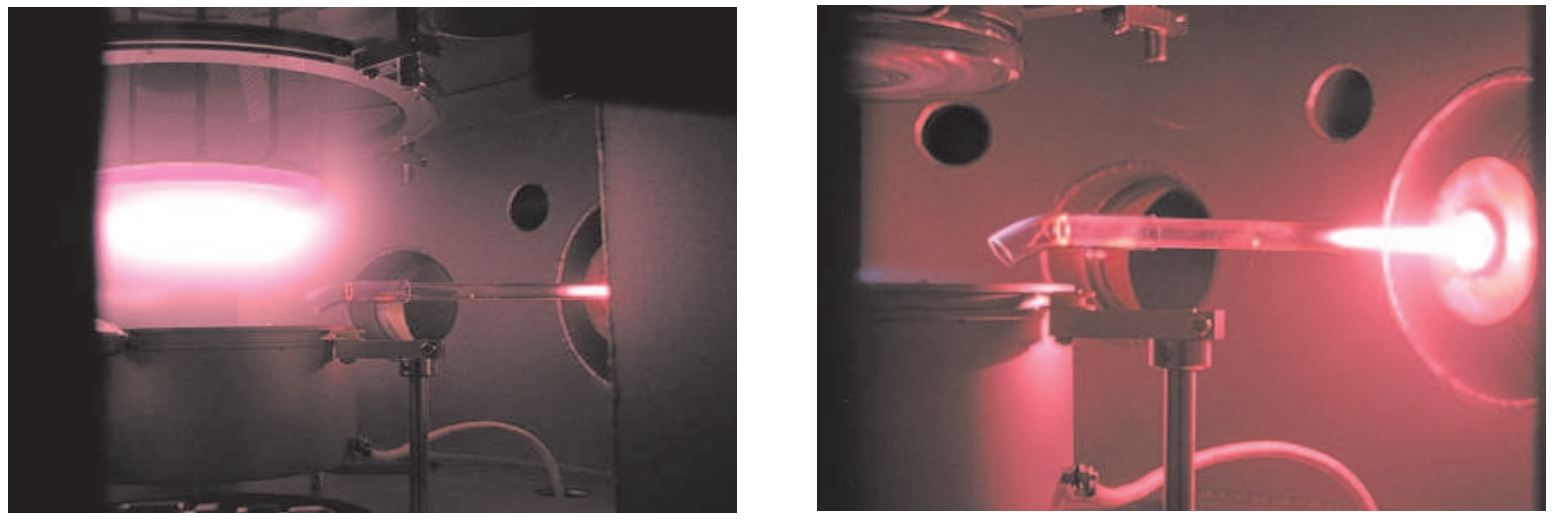

Figure 2.5: The double plasma experimental setup - combination of an ultra high frequency plasma and an inductively coupled plasma. Left photo: IC plasma together with hydrogen plasma; right photo: UHF hydrogen plasma.

gases are let in through the O-ring, mounted around the quartz cylinder, into the reaction chamber. The gas flow is controlled by mass flow controllers (MKS) and is in the range from 5 to $100 \mathrm{sccm}$. The pressure during the operation is controlled by a pressuremeter (MKS Instruments PR 4000) from atmospheric pressure down to $10^{-7}$ mbar. A power from about $50 \mathrm{~W}$ up to $1 \mathrm{~kW}$ is provided by an RF signal generator (Rhode \& Schawrz SMY 02 Signal Generator) and amplified by an amplifier (Bonn Elektronik, 9kHz - $220 \mathrm{MHz}$, max. $4000 \mathrm{~W}$ ) coupled to the discharge via a matching box to minimize the amount of reflected power.

Information about the plasma chemistry is delivered by a rest gas analyzer (RGA, Pfeiffer) which is installed about $30 \mathrm{~cm}$ far from the center of the plasma. The rest gas analyzer is differentially pumped and separated form the main plasma chamber. The pressure in the rest gas analyzer is about $10^{-6}$ mbar for a working pressure in the main plasma chamber of 0.06 mbar. Another plasma spectrometer, the Balzers PPM 421 plasma process monitor (PPM), is installed inside the lower, grounded electrode. While RGS can measure only neutrals, PPM can measure positive and negative ions as well as neutrals.

The formation of dust particles is monitored by measuring the intensity of scattered laser light at $90^{\circ}$ using a He-Ne laser ( $5 \mathrm{~mW}$ power). After scattering, the light is focused with one focal lens and directed to the optical cable, the signal being transmitted further to the optical spectrometer. To obtain the optical spectrum a double monochromator with a resolution of $3 \AA$ in combination with a photomultiplier is used. Particles are also analyzed by means of secondary electron microscopy (SEM) after removal from the chamber. The results of these investigations are presented in chapter 4 , section 4.3. The growth/etching speeds of the films are measured in- 


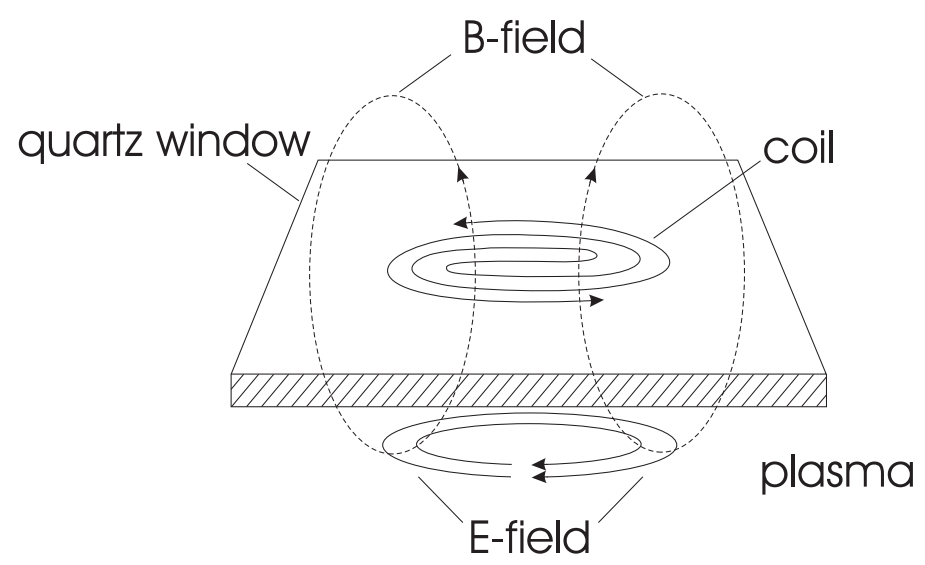

Figure 2.6: Sketch of the electric and magnetic fields induced in a planar coil.

situ. For this purpose a rotating analyzer spectroscopic Woolam M-88 ellipsometer is installed on the chamber. These results are presented in chapter 4 , section 4.4 .

Fig. 2.5 presents two photos of the double plasma experimental setup. The left photo shows the glass tube with the hydrogen plasma (without RF plasma). The right photo shows both plasmas - the inductively coupled RF plasma together with the hydrogen plasma. Also the quartz cylinder with the coil inside, the lower electrode and the O-ring structure for the precursor gas supply can be seen. The hydrogen source is in details described in section 2.3.

\section{Modes in ICP discharges}

In an inductively coupled plasma the RF power is coupled to the plasma via an inductive circuit element which can either be immersed in the plasma or be adjacent to the plasma [13]. In this experimental setup, the coil is adjacent to the plasma on the air side of the quartz window. The RF current flowing through the coil induces an RF magnetic field $(\vec{B})$ which penetrates through the quartz window into the plasma. According to the Faraday's law: $\nabla \times \vec{E}=-\partial \vec{B} / \partial t$ the time-varying RF magnetic field induces a toroidal electric field $(\vec{E})$.

Starting from low values of the applied power, a high potential drop between the powered and the grounded end of the coil exists so that the excitation of the discharge has an electrostatic origin. This potential leads to the capacitive mode of coupling, which is also known as E-mode or dark mode. A plasma operating in this mode is characterized by sheaths of significant thicknesses and low electron density. Generally, the influence of the electric field can be reduced using a Faraday shield.

With the further increase of the RF power the generated magnetic field induces an electric field so that the excitation of the plasma now has an electromagnetic 


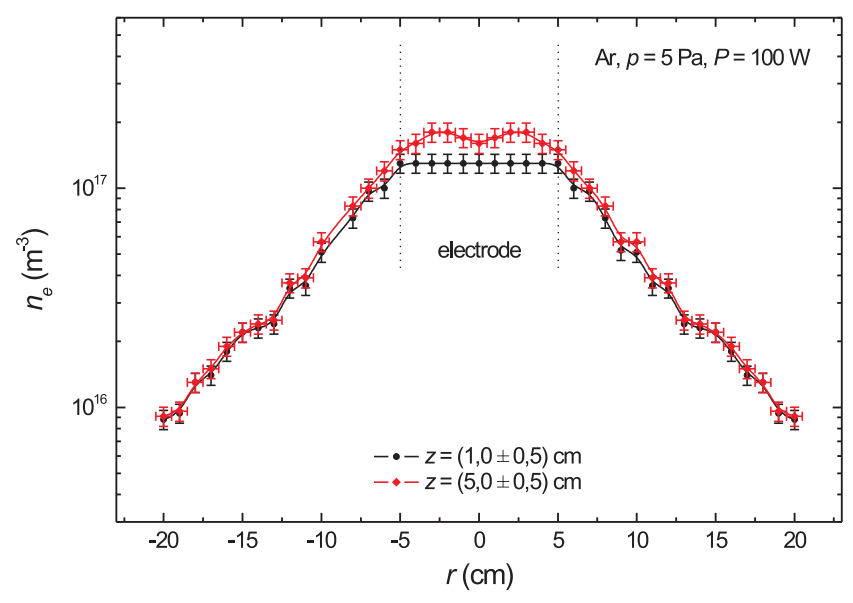

Figure 2.7: The radial profile of the electron density in an Ar plasma for two axial positions $z=(1.0 \pm 0.5) \mathrm{cm}$ and $z=(5.0 \pm 0.5) \mathrm{cm}[15]$.

origin. As the power increases the brightness of the plasma increases too and in one moment suddenly the brightness of the discharge changes. This moment is the onset of the inductive mode, also known as H-mode. The plasma in this mode has an about two orders of magnitude higher electron density than E-mode, and thinner, almost negligible sheath regions. The plasma potential and the mean electron energy are also lower.

In our setup the Faraday shield is not used so this inductively coupled discharge operates in every moment in a mixture of the two modes (capacitive and inductive). In general it is questionable if any inductive discharge operates in an entirely pure inductive mode due to the presence of the high potentials existing on the edges of the coupler.

The radial profile of the electron density in an Ar plasma was measured by Scharwitz [15] for two axial positions $z=(1.0 \pm 0.5) \mathrm{cm}$ and $z=(5.0 \pm 0.5) \mathrm{cm}$ is presented in Fig. 2.7 [15]. Hereby $r=0$ corresponds to the center of the electrode and $z=0$ is on the surface of the lower electrode. At the highest position of $1 \mathrm{~cm}$ above the lower electrode the electron density has a flat profile along the electrode while at 5 $\mathrm{cm}$ it has small recess in the middle and going away form the center of the discharge, two bumps following the profile of the induced electric field inside the plasma.

The graph in Fig. 2.8 presents the axial profile of the normalized electron density at radial position $r=5 \mathrm{~cm}$ (end of the electrode). Measurements are performed in Ar plasma at $p=0.05$ mbar and for an applied power of $P=100 \mathrm{~W}$ [15]. 


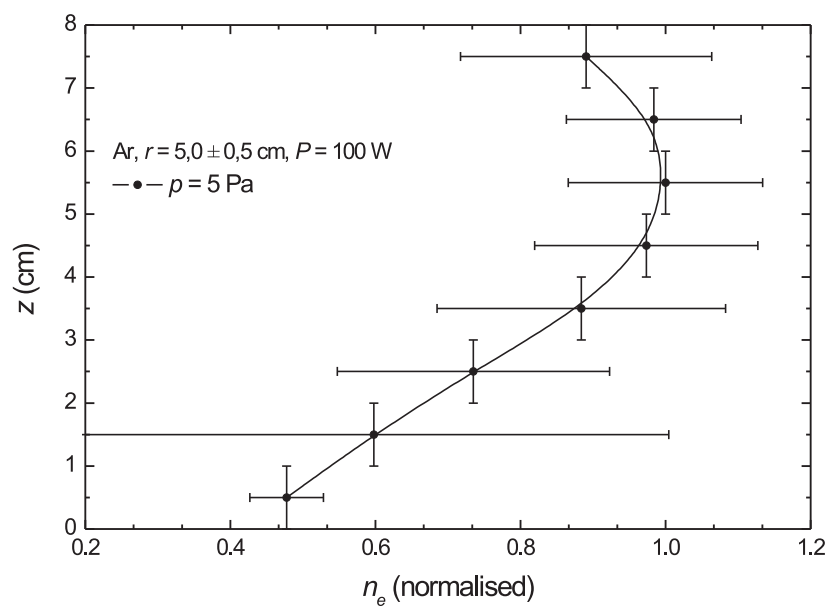

Figure 2.8: The axial profile of normalized electron density in Ar plasma at radial position $r=5 \mathrm{~cm}$ (edge of the electrode) [15].

\subsection{The UHF plasma as source for hydrogen atoms}

The hydrogen atom source produces hydrogen atoms from hydrogen molecules in the discharge being excited by a surfatron working in the UHF regime at $144.1 \mathrm{MHz}$.

The signal coming from an RF generator (Rhode and Schwarz, Signal generator SML 01) is amplified (Bonn Elektronik, Amplifier BTA 0122-500) and supplied to the launcher. The transmitted and the reflected power are measured by a Rhode and Schwarz NRT Power Reflection Meter. The effective power absorbed by the plasma is about $100 \mathrm{~W}$. The molecular hydrogen flow is in the range of 1 to 100 sccm.

To test the production of hydrogen atoms in the glass tube and their injection into the region of expanding plasma, the following experiment is performed. The discharge is ignited in molecular hydrogen and injected into the expanded argon plasma, on the way presented in Fig. 2.9. The hydrogen atoms in the UHF discharge can be created through two pathways:

$$
\mathrm{H}_{2}+\mathrm{e}^{-} \rightarrow \mathrm{H}+\mathrm{H}+\mathrm{e}^{-}
$$

in electron impact dissociation process with the $E_{\text {diss }}=8.9 \mathrm{eV}$ [16] or in the process of dissociative excitation of $\mathrm{H}_{2}$ molecules: 


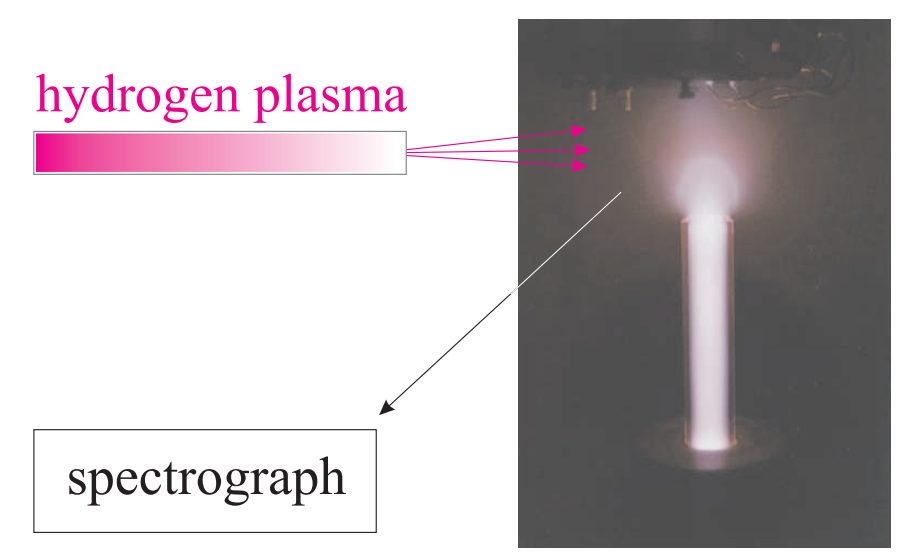

Figure 2.9: Photo of the argon expanding plasma and sketch of the hydrogen atom source showing how optical spectroscopic detection of hydrogen atoms is performed. The light coming from the plasma ball is focused with the lens to the entrance of optical spectrograph and the intensity of the hydrogen atoms $\mathrm{H}_{\alpha}$ line emission is measured.

$$
\mathrm{H}_{2}+\mathrm{e}^{-} \rightarrow \mathrm{H}_{2}^{*}+\mathrm{e}^{-}
$$

and then:

$$
\mathrm{H}_{2}^{*} \rightarrow \mathrm{H}^{*}+\mathrm{H}+\mathrm{E}_{\mathrm{k}}
$$

where $E_{k}$ is the released kinetic energy [17].

The hydrogen atoms, flowing from the tube into the region of the plasma expansion, in collisions with the argon ions and metastables become excited $\mathrm{H}^{*}$ atoms.

$$
\operatorname{Ar}^{+}\left(\mathrm{Ar}^{*}\right)+\mathrm{H} \rightarrow \mathrm{Ar}+\mathrm{H}^{*}
$$

In a radiative process:

$$
\mathrm{H}^{*}(\mathrm{n}=3 \rightarrow \mathrm{n}=2) \rightarrow \mathrm{H}+\mathrm{h} \nu,
$$

the excited $\mathrm{H}^{*}$ atoms deexcite from the $n=3$ to the $n=2$ atomic level and the light coming from the expanding argon plasma ball originating in this transition at a wave length $\lambda=656.3 \mathrm{~nm}$ is focused with the convergent lens to the entrance of the optical spectrograph and the intensity of the resulting Balmer $\mathrm{H}_{\alpha}$ line is measured as a function of the power applied to the hydrogen discharge.

The hydrogen plasma is then turned off while the argon plasma remains on. Molecular hydrogen is injected into the expanding plasma region. The intensity of the $\mathrm{H}_{\alpha}$ line coming from the argon expanding plasma region (due to the dissociation process of molecular hydrogen occurring in that region) and the intensity of the $\mathrm{H}_{\alpha}$ line of 


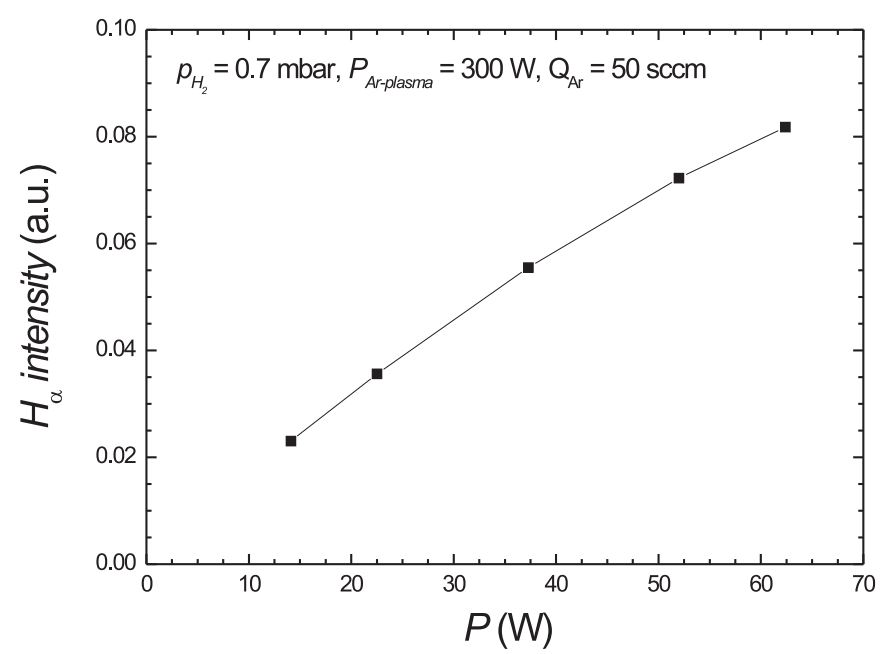

Figure 2.10: Dependence of Balmer-alpha $\left(\mathrm{H}_{\alpha}, n=3 \rightarrow n=2\right.$ atomic transition, $\lambda=$ $656.3 \mathrm{~nm}$ ) line emission on the power applied to the hydrogen discharge.

the reflected light from the chamber wall is measured. The total intensity of the $\mathrm{H}_{\alpha}$ line of the reflected light from the chamber wall and coming from the region of the expanding argon plasma when molecular hydrogen is injected is then subtracted from the total intensity of the $\mathrm{H}_{\alpha}$ line of the light coming from the plasma ball region when hydrogen atoms are injected and of the light reflected from the chamber wall. The obtained intensity of the $\mathrm{H}_{\alpha}$ line represents the intensity of the light coming from the atomic hydrogen source. The behavior of the intensity of the $\mathrm{H}_{\alpha}$ line as a function of the applied power to the hydrogen discharge is presented in Fig. 2.10.

During the experiment it is observed that the effect of the cooling of the discharge tube plays a crucial role in the production of hydrogen atoms. This is demonstrated by the graph in Fig. 2.11. As soon as the air cooling is turned off the intensity of $\mathrm{H}_{\alpha}$ line rapidly decreases, while it increases as soon as the air cooling is turned on. Since the surface recombination of hydrogen atoms on quartz is very sensitive on the temperature of the quartz tube, this effect can be attributed to the increase of recombination of hydrogen atoms on the tube wall when the air cooling is switched off.

The discharge is usually operated in the fully recombining mode in which only quasi-stable neutral particles as $\mathrm{H}^{0}, \mathrm{H}_{2}, \mathrm{H}_{2}^{*}$ are carried by the gas flow into the main chamber. The fully recombining mode is characterized by the absence of charged species and is recognized by the dying out of the luminous plasma region well within the discharge tube what can be seen on the photos presented in Fig. 2.5. 


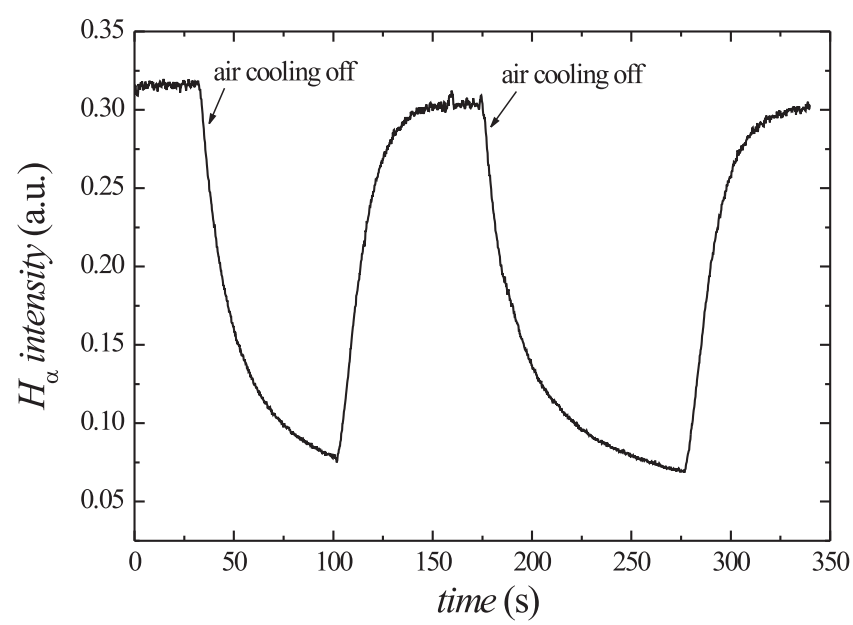

Figure 2.11: Effect of the air cooling on the atomic hydrogen production.

\subsection{Sample preparation and analysis}

Empty semiconductor grade n-doped silicon wafers are used for studying the thin film deposition. Before the deposition process, the wafer is not treated in any way.

Samples used for measuring the film etching speed consist of a wafer coated with a hydrocarbon film. This coating is deposited onto the silicon wafer in a capacitively coupled standard gaseous electronic cell (GEC). The electrodes are $10 \mathrm{~cm}$ in diameter and separated by a distance of $4 \mathrm{~cm}$. The empty wafer is placed on the lower, grounded electrode. In the case of methane films the upper electrode is powered with $40 \mathrm{~W}$ while for acetylene films the power is $20 \mathrm{~W}$. The gas flow is $2 \mathrm{sccm}$ in both cases. The typical thickness of the film is $150-200 \mathrm{~nm}$. The optical constants of the films deposited from methane are around $\widetilde{n}_{0}=1.60-\mathrm{i} \cdot 0.001$ and of the film deposited from acetylene are around $\tilde{n}_{0}=1.75-\mathrm{i} \cdot 0.009$. The thickness and the optical constants are measured ex-situ by means of a rotating analyzer spectroscopic ellipsometer (Woolam M-88) in the wavelength range $270-750 \mathrm{~nm}$ before the samples are used. The film optical constants $n$ and $k$ are determined using the parametric semiconductor model.

In the first double plasma experimental setup (MW/UHF) the etching speed is measured ex-situ and is given by the difference between the thickness before and the thickness after the etching process divided by the time of the film exposure to the plasma. Samples are mounted in the sample holder and the position of the wafer is at $45^{\circ}$ relative to both plasmas. The position of the wafer is presented in Fig. 2.1.

Fig. 2.12 shows the profile of the deposited film along the center line (this line is 

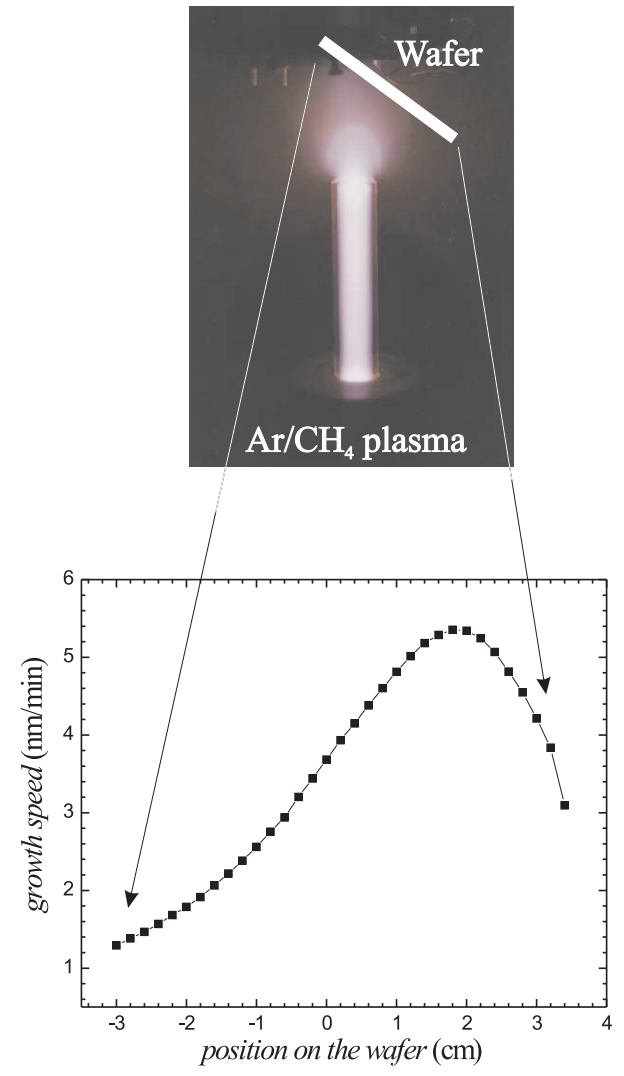

Figure 2.12: The profile of the deposited film along the center line (this line lays in the same plane where two glass cylinders lay) of the wafer. The film profile is not symmetric with respect to the center at $x=0 \mathrm{~cm}$ of the wafer since the wafer has an angle of $45^{\circ}$ with respect to both plasmas.

in the same plane where the two glass cylinders lay) of the wafer. The film growth is not symmetric with respect to the center at $x=0 \mathrm{~cm}$ of the wafer since the wafer has an angle of $45^{\circ}$ with respect to both plasmas.

In the second experiment (ICP/UHF) the deposition/etching speeds are measured in-situ. The samples are placed on the top and in the center of lower electrode during the deposition/etching process.

The growth and the etching speeds of the films are measured and the optical constants are determined in-situ (in the first experimental set up only ex-situ) with the installed rotating analyzer spectroscopic Woolam M-88 ellipsometer. The measurements are performed in the wavelength range of $270-750 \mathrm{~nm}$.

The chemical structure and the bonding within the films are characterized by $e x-$ situ Fourier transform infrared absorption spectroscopy (Bruker FTIR spectrometer) and micro-Raman spectroscopy (spectrometer LabRam series Jobin Yvon Co). 


\section{Chapter 3}

\section{Diagnostics}

In this Chapter the diagnostics used in thin film analysis and characterization and some generalities on them are reviewed. The principles of ellipsometry, infrared spectroscopy, Raman spectroscopy, and their applications for the determination of the thin film properties and characterization of the thin film structure are described. This chapter contains also the basic generalities on mass spectroscopy used in the analysis of the plasma composition during the investigations of dust particle formation and thin amorphous hydrogenated film deposition in inductively coupled discharge. Together with the mass spectrometry scanning electron microscope (SEM) is employed in investigation of the dust particle size and shape. The principle of scanning electron microscopy is not described in this work.

\subsection{Ellipsometry}

\subsubsection{Light polarization}

When the two linearly polarized waves traveling along the same path, with the same frequency but with arbitrary phase or amplitude combine the resultant wave has the elliptical polarization. The concept of the wave elliptical polarization is presented in Fig. 3.1.

The linear polarization and the circular polarization are two special cases of wave polarization. When the two traveling waves are orthogonal and in phase the resultant wave has linear polarization. When the two waves have equal amplitudes and are phase-shifted by $90^{\circ}$, the resultant wave has circular polarization.

\subsubsection{Basic principles of the ellipsometry measurements}

Ellipsometry is a very sensitive optical measurement technique for determining the properties of surfaces and thin films. For probing surfaces and thin films the spec- 


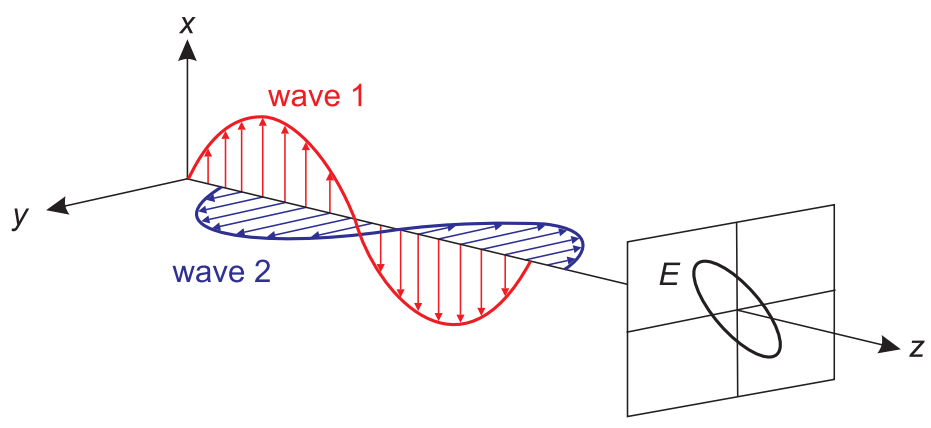

a)

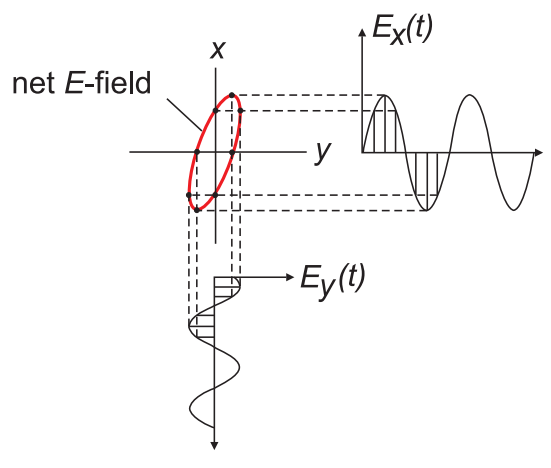

b)

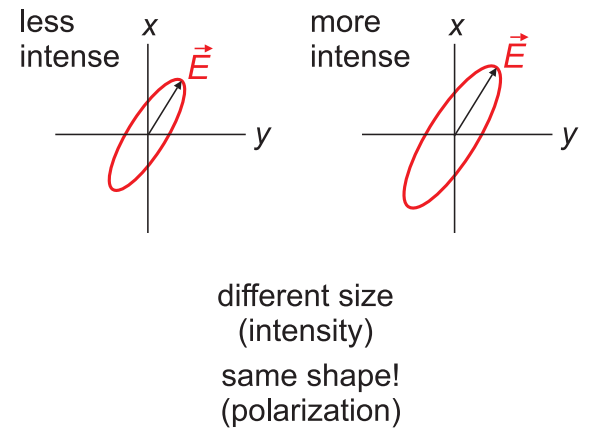

c)

Figure 3.1: a) Combination of two waves with arbitrary phase and amplitude producing an elliptically polarized wave. b) The components of the electric field of the elliptically polarized wave in $x$ and $y$ direction. c) The polarization is described with two numerical values: orientation and ellipticity.

troscopic ellipsometry uses polarized light in the near-UV, visible and near-IR wavelength region.

Figure 3.2 illustrates the basic principles of the ellipsometry measurements. Linearly polarized light beam is directed toward the sample of unknown properties, structure and thickness. The light interacts with the sample and reflects from it. During the interaction with the sample, the light changes its polarization state from linear to elliptical (therefrom the name of the technique). The change in the polarization state of the light beam is then measured and analyzed. In Figure 3.2, the vector of the electric field together with the so-called $s$-direction and $p$-direction is presented. These directions are commonly used in ellipsometry and, together with the direction of the light propagation, define the right-hand Cartesian coordinates.

The $p$-direction lies in the plane of incidence ( $\mathrm{p}$ from germ. Parallel - parallel). The plane of incidence is the plane perpendicular to the surface of the sample and is defined by the incident light and the direction normal to the surface. The s-direction 


\section{linearly polarized light}

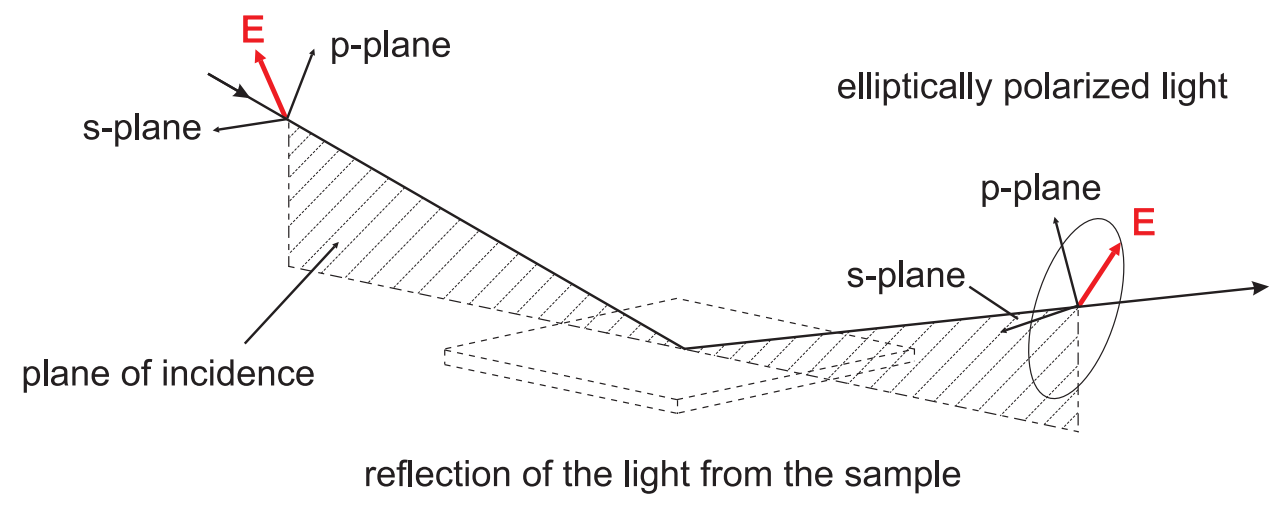

Figure 3.2: Basic measurement geometry of ellipsometric experiment. Incident light beam with known polarization state interacts with the sample of unknown thickness and properties and reflects. Reflected light beam is then elliptically polarized (after [18]).

(s from germ. Senkrecht - perpendicular) is perpendicular to the plane of incidence.

The $p$-wave and the $s$-wave are not necessarily in phase. After reflection, generally, there is a phase shift which is not necessarily the same for both waves. If $\delta_{1}$ is the phase difference between the $p$-wave and the $s$-wave before the reflection and $\delta_{2}$ is the phase difference after the reflection, then the relative phase shift $\Delta$ induced by the reflection is [19]:

$$
\Delta=\delta_{1}-\delta_{2}
$$

The values of $\Delta$ lie in the range from $-180^{\circ}$ to $+180^{\circ}$.

The reflection induces also an amplitude reduction for both the $p$-wave and the $s$-wave and generally it can be different for these two waves. The total reflection coefficient for $p$-wave and $s$-wave is the ratio of the outgoing wave amplitude to the incoming wave amplitude. $\left|R^{p}\right|$ and $\left|R^{s}\right|$ are the magnitudes of these reductions. The ratio of these two magnitudes is:

$$
\tan \Psi=\frac{\left|R^{p}\right|}{\left|R^{s}\right|},
$$

where $\Psi$ is the angle whose tangent is the ratio of the magnitudes of the total reflection coefficients. This is a real number and its value ranges between $0^{\circ}$ and $90^{\circ}$. The ratio $\rho$ of the total reflection coefficients:

$$
\rho=\frac{R_{p}}{R_{s}},
$$

is a complex number. 
The connection between $\Psi$ and $\Delta$ is:

$$
\rho=\tan \Psi \cdot e^{i \Delta}
$$

This is the so-called fundamental equation of ellipsometry. The magnitude of $\rho$ is contained in $\tan \Psi$ and the phase of $\rho$ is contained in the exponential term $e^{i \Delta}$. The ellipsometer measures $\Delta$ and $\Psi$, while the quantities of interest as thickness, optical constants, etc. are calculated based on the used model.

\subsubsection{Ellipsometer configuration}

In Fig. 3.3 the basic principles of the ellipsometry measurements are presented on the example of the rotating analyzer ellipsometer (RAE). An arc lamp or a laser is employed as a light source in a spectroscopic ellipsometer. Generally, before the light enters into the polarizer it is unpolarized. A polarizer is an optical element which converts the initial unpolarized light beam into a light beam with known polarization. In this setup the position of the polarizer is held fixed. Very often linear polarizers are used. The polarization state of the light before it will reach the substrate is linear (position A). After the reflection from the substrate, the light becomes elliptically polarized (position B) and goes in the analyzer, a second polarizer where the light beam polarization state is resolved. The analyzer rotates with a speed of $10-60 \mathrm{~Hz}$. After passing through the analyzer the light is again linearly polarized (position $\mathrm{C}$ ) and is directed to detector. The detector detects the direction of polarization and the amplitude (actually intensity because amplitude follows the analyzer rotation) which vary during the time. Three types of detectors are used in ellipsometry: photomultipliers tubes, semiconductor photodiodes and CCD (charged-coupled device).

\subsubsection{Optical constants}

In a classical approach with a Lorentz model, following the descriptions given in [18], for an oscillator the dielectric function of matter, $\widetilde{\epsilon}$, can be written in the form:

$$
\widetilde{\epsilon}=1+\frac{4 \pi e^{2}}{m} \sum_{j} \frac{N_{j}}{\left(\omega_{j}^{2}-\omega^{2}\right)-i \Gamma_{j} \omega},
$$

where $e$ and $m$ are the charge and the mass of an electron, $N_{j}$ the number density of oscillators with the resonant frequency $\omega_{j}, i$ the imaginary unit, $\Gamma_{j}$ is the damping factor, and $\sum_{j} N_{j}=N$ is the total number of oscillators. The dielectric function and the complex index of refraction are connected by the relationship: 


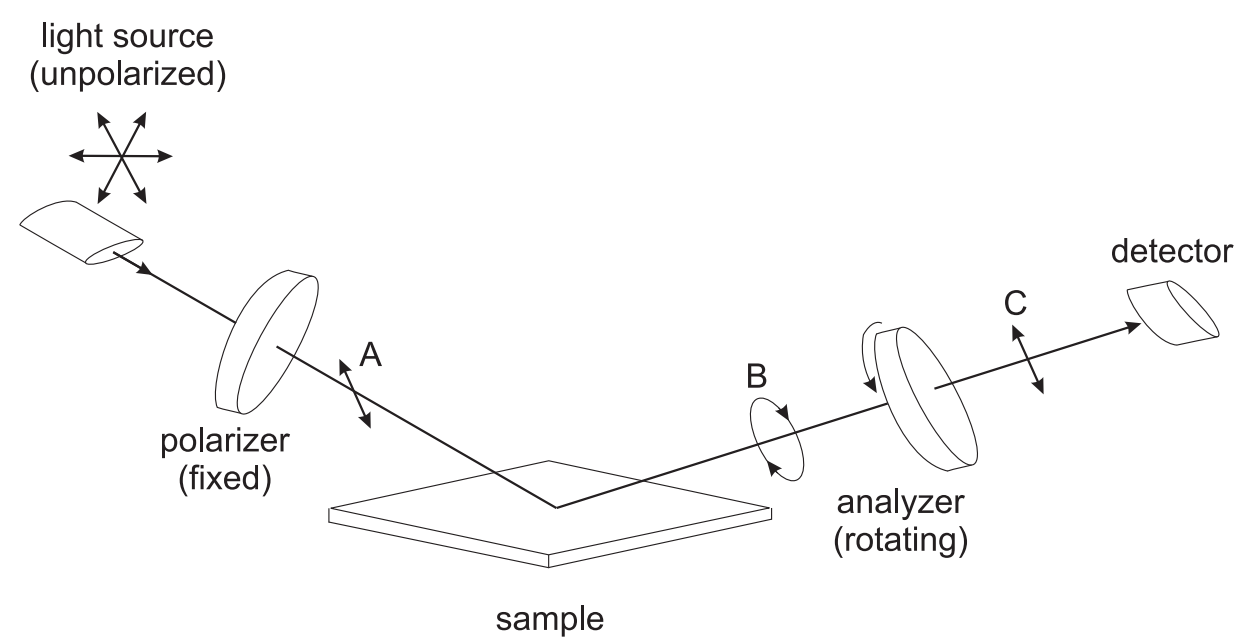

Figure 3.3: Schematic representation of a rotating analyzer ellipsometer (after [18]).

$$
\widetilde{\epsilon}=\widetilde{N}^{2}
$$

The dielectric function, $\widetilde{\epsilon}$, represents the degree to which the material can be polarized, thus it describes its interaction with an electromagnetic wave. As a complex number, the dielectric function can be expressed by the relationship:

$$
\widetilde{\epsilon}=\epsilon_{1}(\lambda)+i \epsilon_{2}(\lambda) .
$$

where $\epsilon_{1}$ is the volume polarization term for induced dipoles and $\epsilon_{2}$ is the volume absorption.

The index of refraction is a complex complex number too, and can be expressed as:

$$
\tilde{n}=n(\lambda)+i k(\lambda)
$$

The relationship between $n$ and $k$ (the real and the imaginary part of the complex index of refraction) and $\epsilon_{1}$ and $\epsilon_{2}$ (the real and the imaginary part of the complex dielectric function) can be obtained from equations (3.6) - (3.8):

$$
\begin{aligned}
& n=\sqrt{\frac{1}{2}\left[\left(\epsilon_{1}^{2}+\epsilon_{2}^{2}\right)^{\frac{1}{2}}+\epsilon_{1}\right]}, \\
& k=\sqrt{\frac{1}{2}\left[\left(\epsilon_{1}^{2}+\epsilon_{2}^{2}\right)^{\frac{1}{2}}-\epsilon_{1}\right]} .
\end{aligned}
$$

While $n$ and $k$ describe what the material does to the light, $\epsilon_{1}$ and $\epsilon_{2}$ describe what the light does to the material. 
The index of refraction (or refractive index) gives the change of the propagation speed of the electromagnetic wave in a material comparing with the speed of the wave in vacuum. The extinction coefficient is related to the energy loss of the wave due to the absorption in the material.

The index of refraction and the extinction coefficient are quantities connected by the Kramers-Kronig relation [18]:

$$
\begin{gathered}
n(E)-1=\frac{2}{\pi} \cdot P \cdot \int_{0}^{\infty} \frac{k\left(E^{\prime}\right) E^{\prime}}{E^{\prime 2}-E^{2}} \cdot d E^{\prime}, \\
k(E)-1=-\frac{2}{\pi} \cdot P \cdot \int_{0}^{\infty} \frac{E \cdot n\left(E^{\prime}\right)}{E^{2}-E^{2}} \cdot d E^{\prime} .
\end{gathered}
$$

The integrals in equations (3.11) and (3.12) are Cauchy integrals and $P$ is the Cauchy principal value. These relationships show that if the value of the extinction coefficient is known over the entire energy range the index of refraction can be derived using the Kramers-Kronig relation and vice versa. The relations which satisfy the Kramers-Kronig relation are Kramers-Kronig consistent relations.

\subsection{Infrared and Raman spectroscopy}

The electromagnetic waves in the ultraviolet (UV) to visible spectral range are able to excite vibrational motions of the chemical bonds between atoms or atoms and functional groups which are parts of molecules in a gas phase or in a liquid or solid material. Infrared and Raman spectroscopy are based on the analytical techniques used for analyzing the sample of unknown structure. The interpretation of both infrared and Raman spectra is based on the comparison of the spectrum acquired from the sample of unknown structure and the known frequencies of the functional groups. The analysis of vibration of any structure is established on the degrees of freedom that structure possesses.

At any given time a molecule possesses energy which is the sum of translational, rotational, vibrational, and electronic energy:

$$
E_{\text {total }}=E_{\text {translational }}+E_{\text {rotational }}+E_{\text {vibrational }}+E_{\text {electronic }} .
$$

The translational energy $E_{\text {translational }}$ is related to the movement of the molecule through the space, which is a consequence of the normal thermal motion of particles. The rotational energy $E_{\text {rotational }}$ arises from the rotation of the molecule around some of its axes. The vibrational energy $E_{\text {vibrational }}$ arises from vibrations of the atoms inside the molecule around their equilibrium positions. $E_{\text {electronic }}$ corresponds to the 


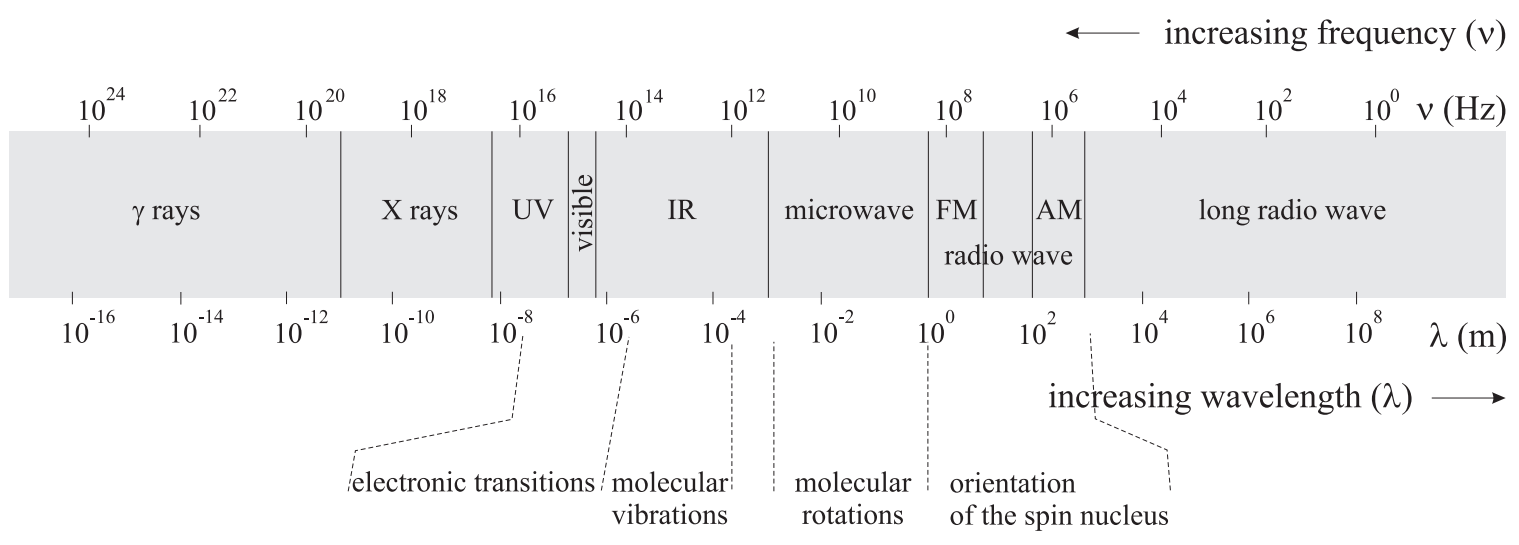

Figure 3.4: The electromagnetic spectrum and its conditional classification in different regions according to frequency $(\nu)$ or wavelength $(\lambda)$.

motion of electrons in the approximation of fixed nuclear configuration or BornOppenheimer approximation. This approximation is valid when the interactions or the coupling between different forms of motion in molecule can be neglected. The vibrational and rotational transitions can be studied by infrared and Raman spectroscopy.

A molecule consists of two or more atoms. Each atom has three degrees of freedom which correspond to motions along the three axes $(x, y, z)$ in the Cartesian coordinate system. A molecule consisting of $n$ atoms has $3 n$ degrees of freedom. Three degrees describe the translational motion of the whole molecule, 3 degrees describe the rotational motion of the entire molecule and the remaining $3 n-6$ degrees of freedom describe the vibrational motion (fundamental vibrational modes or normal modes of vibration) of a nonlinear molecule. If the molecule is linear it has 2 degrees of freedom describing the rotational motion and therefore has $3 n-5$ fundamental vibrations. From these modes the number of stretching vibrations is $n$-1 and the number of bending vibrations is $2 n-5$. The vibration frequencies of most of the molecules lie in the infrared region of the electromagnetic spectrum. The vibrational frequencies of most of the molecules which produce a net change in the dipole moment of a molecule will appear as infrared active. Those vibrations which induce polarizability changes in the molecule will appear as Raman active. Some of the vibrations can be both infrared and Raman active.

The infrared electromagnetic region can be divided into three sub regions: the near IR (14000-4000 $\mathrm{cm}^{-1}$ ), mid IR (4000-400 $\mathrm{cm}^{-1}$ ) and far IR (approximately 400-10 $\left.\mathrm{cm}^{-1}\right)$. The waves from near infrared region can excite overtone bands, waves from mid infrared can be used for investigation of vibrational transitions and associated rotational-vibrational molecule structure, while the waves from far IR region can be 
used for investigations of rotational transitions.

The positions of spectral peaks appearing due to absorption can be expressed either in wavenumbers $(\tilde{\nu})$ (the number of oscillations per unit length) or in wavelengths $(\lambda)$. The relation between them is:

$$
\tilde{\nu}\left(\mathrm{cm}^{-1}\right)=\frac{1}{\lambda(\mu m)} \cdot 10^{4}
$$

\subsubsection{Infrared spectroscopy}

The following description of infrared and Raman spectroscopy is according to the traditional picture. The modern description is more complicated utilizing quantum mechanics. The traditional picture suffice have o describe the generalities of the technique.

When the exposure of an atomic system to electromagnetic radiation is accompanied by a change in the dipole moment, the system will absorb or emit some amount of radiation. Charges in a molecule undergo a periodic vibrational motion so that the dipole moment changes also periodically. The frequencies of the emitted or the absorbed radiation from the molecule are normal frequencies.

The dipole moment is a vector quantity directed from the center of gravity of protons to the center of gravity of electrons in a molecule. In the case of a simple dipole, the dipole moment $\vec{p}$ is defined as the product of one of charges, $q$, and the charge separation, $\vec{r}$ :

$$
\vec{p}=q \cdot \vec{r}
$$

In the case of a complex molecule the positive particle can represent the sum of all positive charges (protons) in the molecule (and is set at their center of gravity), while the negative particle can represent the sum of all negative charges, i.e. electrons.

The change in the dipole moment can originate only due to the change of its direction. In symmetric molecules - molecules with a center of symmetry - infrared active and inactive modes can appear. For example, $\mathrm{H}_{2}, \mathrm{H}_{2} \mathrm{O}$ and $\mathrm{CO}_{2}$ molecules are all symmetric molecules. During the vibration the $\mathrm{H}_{2}$ molecule will retain its center of symmetry so there will be no change in the dipole moment. On the other hand, in a linear $\mathrm{CO}_{2}$ molecule three vibrational modes are possible. During the symmetrical, in-phase vibrations of the $\mathrm{CO}_{2}$ molecule, the $\mathrm{O}$ molecules approach and move away from the $\mathrm{C}$ molecule without moving of the center of symmetry. This vibrational mode will not appear in the infrared spectrum, being infrared inactive. Asymmetrical motion, out-of-phase stretching and bending vibrations of $\mathrm{O}$ and $\mathrm{C}$ 

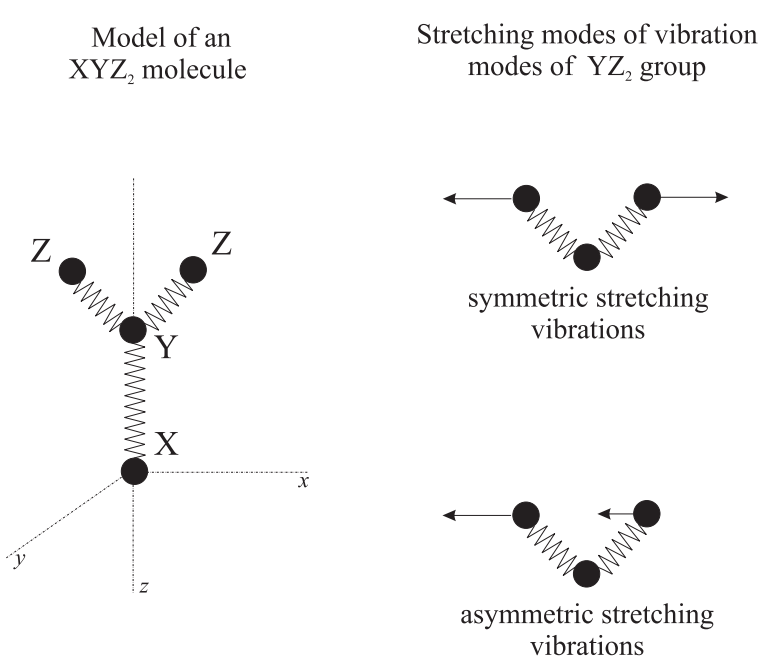
Deformation or bending of vibration
modes of $\mathrm{YZ}_{2}$ group

in-plane deformations
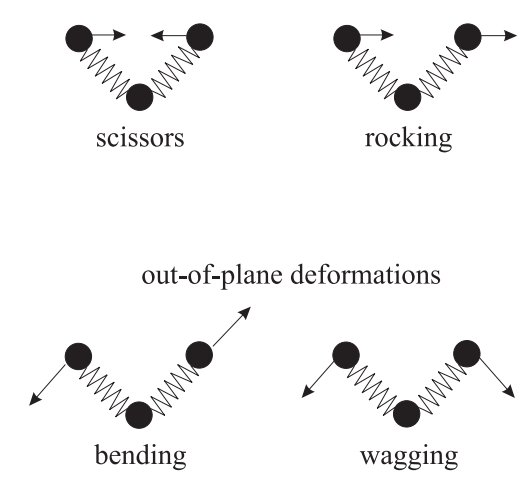

Figure 3.5: Possible stretching and deformation or bending vibrational modes of the $Y Z_{2}$ group in the $X Y Z_{2}$ molecule.

atoms will not retain the center of symmetry, so they will produce a change of the dipole moment and will appear in the infrared spectrum. These two vibrational modes are infrared active modes. The $\mathrm{H}_{2} \mathrm{O}$ molecule is symmetric and nonlinear and all three possible normal vibrations are infrared active. In asymmetrical molecules, such as $\mathrm{HCl}$ or $\mathrm{HCN}$, with permanent dipole moment all vibrational modes are infrared active.

In asymmetrical molecules all normal vibrations are accompanied by a change in the dipole moment so all those vibrations are infrared active. The infrared inactive or active modes can appear in symmetric molecules depending on whether the center of symmetry changes or not during the vibration, respectively.

From the previous explanations it is obvious that the absorption frequency depends on the molecular vibrational frequency while the intensity of the absorbed radiation depends on how effectively the energy is transferred to the molecule. The last depends on the change in dipole moment that occurs as a result of molecular vibrations. The origin of the absorption bends can appear from either the functional groups $\left(\mathrm{C}=\mathrm{O}, \mathrm{C}=\mathrm{N}, \mathrm{C}=\mathrm{C},-\mathrm{CH}_{2}-\right)$ or from the skeletal groups i.e. molecular backbone or skeleton of the molecule $(\mathrm{C}-\mathrm{C}-\mathrm{C})$. Absorption bends can arise from the bond-length changes being than stretching vibrations or from the bond-angle changes being deformation (banding) vibrations. As an illustration for this stretching and deformation modes the $\mathrm{YZ}_{2}$ group in the $\mathrm{XYZ}_{2}$ molecule are presented in Fig. 3.5.

Since the stretching and compressing of the bonds require more energy then the deformation, stretching will occur on higher frequencies than the deformation. Over- 


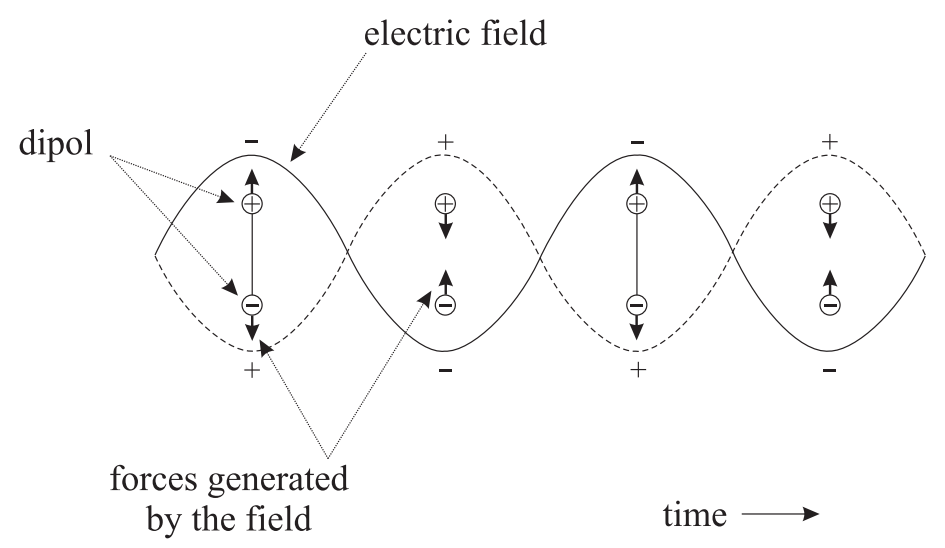

Figure 3.6: When an electromagnetic wave strikes the molecule with a dipole moment, the oscillating electric field of the wave generates forces which cause the dipole to vibrate by alternately stretching and compressing (The figure is taken from Ref. [20]).

tone and combination bands are other two different types of absorption bands which can be observed in the spectrum. Overtone bands appear at about twice the frequency of the fundamental absorption band are therefore difficult to be observed. Combination bands appear as a combination of the fundamental frequencies.

The electric field of the electromagnetic wave will affect the charge distribution in a molecule and exert oppositely directed forces on the electrons and protons during the irradiation. As a result charges are displaced relative to each other and the polarized molecule has an induced dipole moment caused by the external electric field. The oscillation of the electric field of the electromagnetic wave therefore will exert forces which will force the induced dipole moment to oscillate at the frequency of the electromagnetic wave. At a certain frequency those oscillations are simultaneous. The vibrational frequencies where the vibration results in the change of the dipole moment are molecular vibrations.

\section{Infrared detection of different bonds}

The fundamental stretching frequency can be calculated by Hooke's law:

$$
\nu=\frac{1}{2 \pi} \cdot \sqrt{\frac{k \cdot\left(m_{1}+m_{2}\right)}{m_{1} \cdot m_{2}}} .
$$

According to this expression the frequency of the vibration, $\nu$, is related to the masses of the interacting atoms $m_{1}$ and $m_{2}$, and to the force constant $k$ - the strength of the covalent bond between two atoms. The ratio of masses: $m_{1} \cdot m_{2} /\left(m_{1}+m_{2}\right)=\mu$ is known as the reduced mass of a two body system. According to this relation as the force constant $k$ increases, i.e. as the bond is stronger, the vibration frequency 
is higher (wavenumber is lower), and as the atom is lighter the vibration frequency is lower. A good illustration for this can be given by the comparison of single $\mathrm{C}-\mathrm{C}$, double $\mathrm{C}=\mathrm{C}$ and triple $\mathrm{C} \equiv \mathrm{C}$ bonds between two carbon atoms. Their strength is $83 \mathrm{kcal} \cdot \mathrm{mol}^{-1}, 146 \mathrm{kcal} \cdot \mathrm{mol}^{-1}$ and $200 \mathrm{kcal} \cdot \mathrm{mol}^{-1}$ respectively, so these bonds appear in the infrared spectrum at the wavenumbers $\sim 1200 \mathrm{~cm}^{-1}, \sim 1650 \mathrm{~cm}^{-1}$, and $\sim 2100 \mathrm{~cm}^{-1}$, respectively. Even though the difference in the bond strength $\mathrm{C}-$ $\mathrm{C}$ and $\mathrm{C}-\mathrm{H}$ is only $16 \mathrm{kcal} \cdot \mathrm{mol}^{-1}\left(99 \mathrm{kcal} \cdot \mathrm{mol}^{-1}\right.$ against $\left.83 \mathrm{kcal} \cdot \mathrm{mol}^{-1}\right)$ the peaks belonging to these bonds will appear at different wavenumbers in the spectrum: $\sim 1200 \mathrm{~cm}^{-1}$ and $\sim 3000 \mathrm{~cm}^{-1}$, respectively, due to the mass difference $\left(m_{H}=1\right.$ amu, $\left.m_{C}=12 \mathrm{amu}\right)$.

The energy curve of such a simple harmonic oscillator is presented in Fig. 3.7 on the left graph. In the harmonic oscillator approximation, the energy levels are equidistant and the molecule can absorb any amount of energy equal to a multiple of the energy difference between energy levels. The anharmonic nature of the molecule is well represented by the empirical Morse's function:

$$
V=D_{e} \cdot\left(1-e^{-\beta\left(r-r_{e}\right)}\right)^{2},
$$

where $V$ is the potential energy, $D_{e}$ is the dissociative energy of the molecule measured from the minimum on the curve, $\beta$ is a constant, and $r_{e}$ is the equilibrium spacing between atoms corresponding to a minimum of the potential energy. The potential energy and the energy levels for anharmonic oscillator are presented on the right graph in Fig. 3.7.

The corresponding vibrational energy of a diatomic molecule in the harmonic oscillator approximation is given by:

$$
E(v)=h \cdot \nu \cdot\left(\frac{1}{2}+v\right),
$$

where $\nu$ is given by equation 4.4. This equation is valid for the lower energy levels, near the minimum in the curve of the potential energy. For the higher vibrational levels the effects of the anharmonicity have to be taken into account.

In the anharmonic oscillator approximation the energy levels are not equidistant and as the atoms move away the energy levels become more closely spaced. Thus, the allowed transitions, $h \nu$, between successive energy levels with increasing $v$ are influenced by the anharmonicity and they require less and less energy. These transitions which occur at $2 h \nu, 3 h \nu, \ldots$ can also be observed in the spectrum. They are overtones and as higher the overtone is the weaker is its intensity in the spectrum. Atoms are separated after the intermolecular distance at which the energy reaches its maximum. This energy corresponds to the dissociation energy of the molecule. 

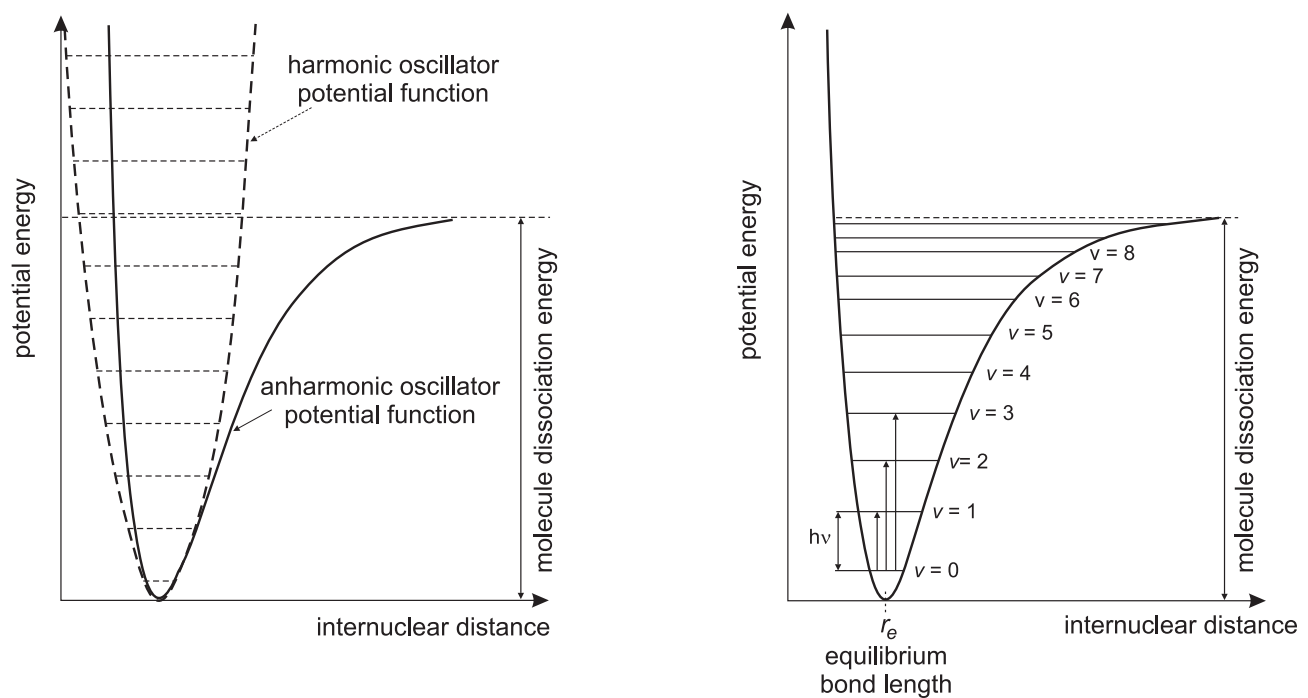

Figure 3.7: The potential energy of a diatomic molecule as a function of internuclear distance. Left gaph: the harmonic (dotted line) and the anharmonic (full line) potential function of oscillator. Right graph: vibrational energy levels of an anharmonic oscillator.

The transition from the ground state to the first vibrationally excited state is not affected by the anharmonicity of the molecule.

\section{Population of energy levels}

The relative population of energy levels in an assembly of a large number of diatomic molecules is given at temperature $T$ by the Boltzmann distribution function:

$$
n_{\text {excited }} / n_{\text {ground }}=e^{-\Delta E / k T}
$$

where $n_{\text {excited }}$ is the number of molecules in the excited state, $n_{\text {ground }}$ is the number of molecules in the ground state, $\Delta E$ is the energy difference between the ground state and the excite state, $k=1.38 \cdot 10^{-23} J \cdot K^{-1}$ is the Boltzmann constant, and $\mathrm{T}$ is the absolute temperature.

\subsubsection{Raman spectroscopy}

The indian scientist Chandrasekhara Venkata Raman described a new type of scattering in 1928 (together with K. S. Krishnan and independently from them G. Landsberg and L. Mandelstam) which has been theoretically predicted before by Smekal.

In Raman spectroscopy infrared or visible laser light with known frequency $\left(\nu_{0}\right)$ is used for the irradiation of a sample of unknown structure. When during the interaction between the incident photons and the sample the molecules rotational 

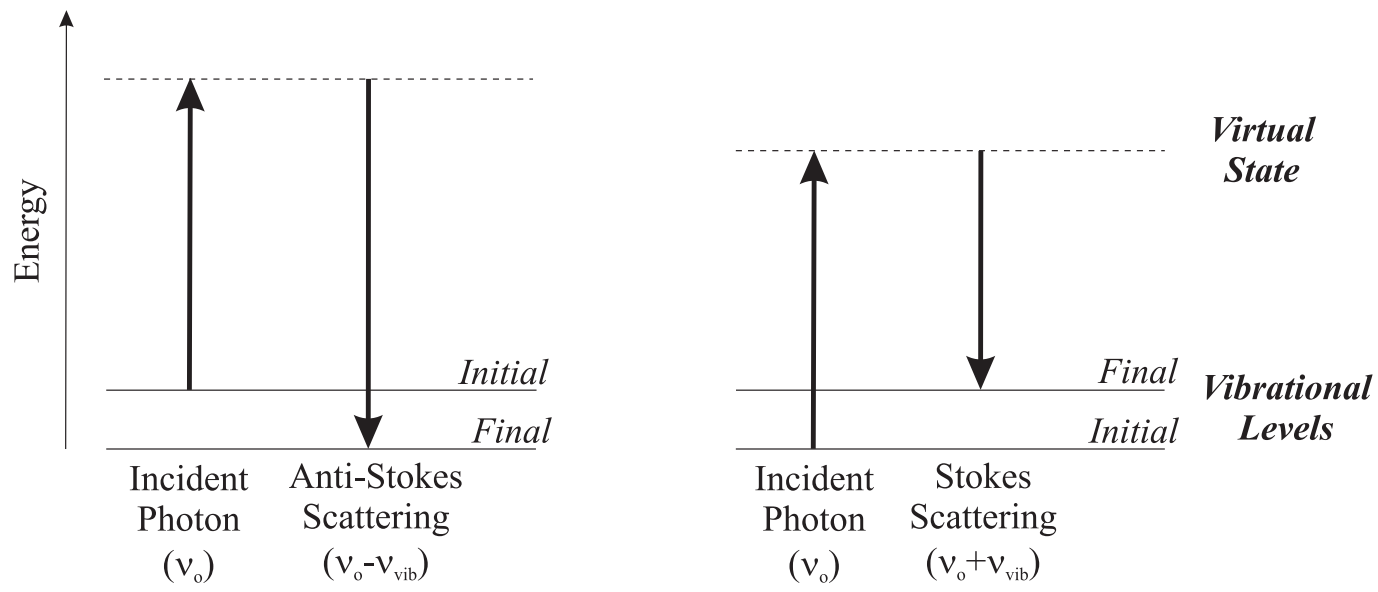

Figure 3.8: When an external electromagnetic radiation of frequency $\nu_{0}$ irradiates the sample, molecules in the sample are excited to additionally vibrate. The scattered light then contains the frequencies $\nu_{0}$ (Rayleigh scattering), $\nu_{0}-\nu_{v i b}$ (Stokes Raman scattering) and $\nu_{0}+\nu_{v i b}$ (anti-Stokes Raman scattering). The difference in energy between incident and scattered photons, i.e. the loss/gain in energy, are presented with the different lengths of the arrows.

and vibrational energy of the molecules is not changed then the scattered photons have the same energy and frequency as the incident photons so they are elastically scattered. This is also known as Rayleigh scattering and is the strongest component in the scattered radiation. After the interaction with the sample molecules a small fraction of the incident photons (approximately $10^{-7}$ ) is scattered at frequencies which differ from the frequency of the initial photons. This is due to the inelastic collision of incident photons with the sample molecules. Incident photons can either lose or gain energy in the interaction with vibrationally/rotationally excited molecules. Thus, the photon energy changes as follows:

$$
\Delta E=h \nu_{i}-h \nu_{s}
$$

and can be positive, if the initial photons gain energy, or negative if they lose energy. The indices $i$ and $s$ designate initial and scattered photons, respectively. Therefore, besides the elastically scattered photons, two groups of photons with different frequencies can be observed.

The group of photons which interacted with the molecules in the ground state has a frequency $\nu_{0}+\nu_{v i b}$ contributing to Stokes Raman scattering and the other group which interacted with the vibrationally excited molecules has $\nu_{0}-\nu_{v i b}$ giving a contribution to anti-Stokes Raman scattering, where $\nu_{v i b}$ is the frequency of the certain vibrational state. This is schematically presented in Fig. 3.8. 
In order that one mode appears as an active mode in the Raman spectrum the applied electric field has to induce a dipole moment in the molecule - the positive charge has to be displaced relative to the negative charge. The induced dipole moment is given by:

$$
\vec{p}=\alpha \cdot \vec{E}
$$

with $\vec{p}$ being the induced dipole moment, $\alpha$ the polarizability and $\vec{E}$ the electric vector of the electromagnetic radiation of frequency $\nu_{0}$. The polarizability is the ability of the electrons to follow the changes induced by the external electromagnetic field. Therefore, one molecular vibration is Raman active if the vibration of the molecule is accompanied by the change of the molecule polarizability.

The relation (3.21) is very general and holds for any direction of the applied electromagnetic field. However, the polarizability can be different in $x, y$ or $z$ direction for different directions of the electric field $\vec{E}$. Then the induced dipole moment, $\vec{p}$, has different values in different directions. This is the case of the anisotropic molecules - where the induced dipole moment and the external electric field - regardless to the orientation of the molecule - have not the same direction. In isotropic molecules the external electric field and the induced dipole moment always have the same direction. From this reason the polarizability is a tensor quantity. The polarizability tensor is symmetric tensor $\left(\alpha_{x y}=\alpha_{y x}, \alpha_{y z}=\alpha_{z y}, \alpha_{x z}=\alpha_{z x}\right)$. The selection rule for one vibrational mode to be Raman active, i.e. to appear in the Raman spectrum, is that at least one of the components of the polarizability tensor is different from zero:

$$
\frac{\partial \alpha_{i, j}}{\partial i} \neq 0,(i, j=x, y, z)
$$

The intensity of the Raman scattered wave is very weak $\sim 10^{-5}$ in comparison with the intensity of the incident laser light. At room temperature the population of the vibrationally excited states is very low and the majority of the molecules are in the ground state. The population of vibrationally excited states is given by the Boltzmann distribution function (3.19). Since most of the molecules at room temperature are in the ground state and only a minority is in the vibrationally excited states, the lines in the Stokes Raman spectrum are stronger then the lines in the anti-Stokes Raman spectrum. 

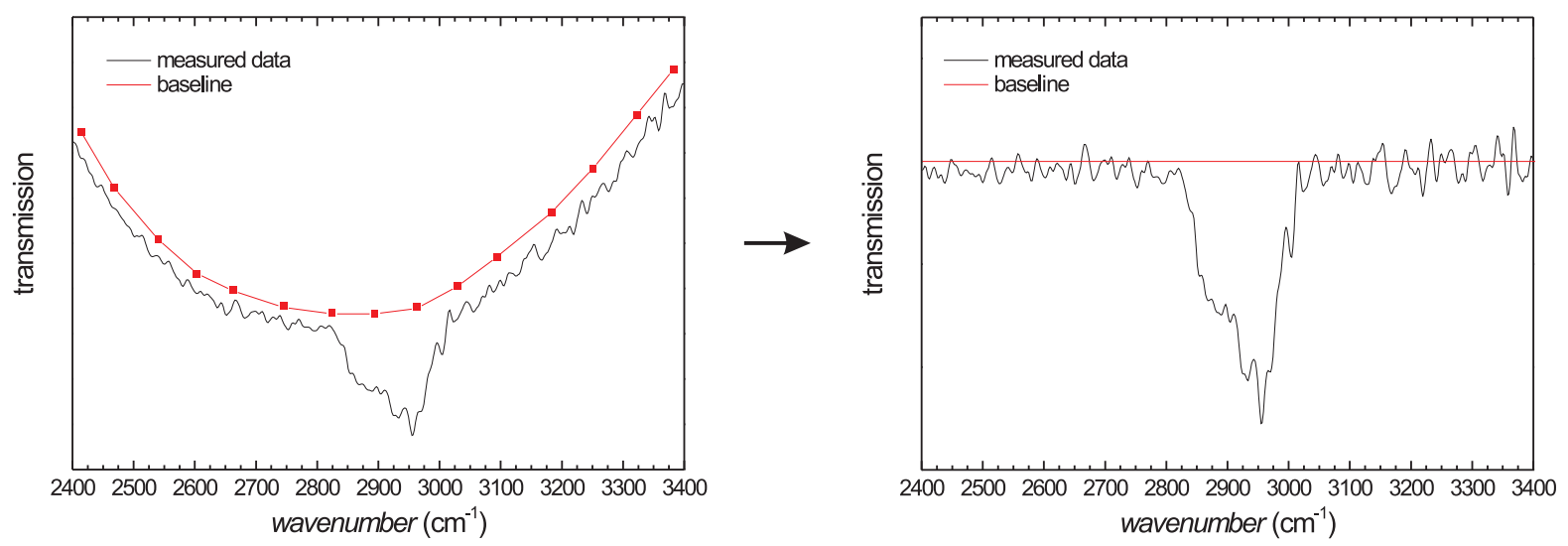

Figure 3.9: An example of measured and baseline corrected infrared spectra. Left graph: measured spectra and baseline; right graph: after-correction.

\subsubsection{Spectra manipulation}

The first step in evaluating the Raman/infrared spectra is a baseline correction since most spectra are more or less tilted and curved. A line as much parallel to the non-peak part of the measured spectrum is drawn and then subtracted from the spectrum. This manipulation of the spectrum improves its appearance but does not change the values of the frequency (wavenumber) of the absorption bands. An example of the performance of such a baseline correction of the spectrum is presented on the infrared spectrum of a:C-H film in Fig. 3.9.

The next step is the deconvolution of the spectrum into individual peaks. The $\mathrm{C}-\mathrm{H}$ IR stretching region can be separated into three subregions: the $s p^{1} \mathrm{C}-\mathrm{H}$ stretching mode is centered on $3300 \mathrm{~cm}^{-1}$, the $s p^{2} \mathrm{C}-\mathrm{H}_{\mathrm{n}}$ modes lie from 2975 to $3085 \mathrm{~cm}^{-1}$, and $s p^{3} \mathrm{C}-\mathrm{H}_{\mathrm{n}}$ lie from 2850 to $2955 \mathrm{~cm}^{-1}$ [21, 22, 23]. Fitting of the $\mathrm{C}-\mathrm{H}$ stretching bands is usually performed with Gaussian curves. Heitz et al showed that for the decomposition of this spectral region the minimum number of bands is five [21]. Each peak in IR spectrum is fitted by a Gaussian function according to $[21,23,24]$. The measured and after that the baseline corrected infrared spectrum is presented in Fig. 3.9.

Due to unknown oscillator strengths for each $\mathrm{CH}_{n}$ unit the infrared spectra are not used for a quantitative calculation of the hydrogen content and distribution but only for observing the qualitative spectral changes $[25,26]$ due to different microstructures. Basically two mistakes can be made. One mistake is that only bonded hydrogen can be detected. Since non bonded hydrogen is not accounted for the to- 

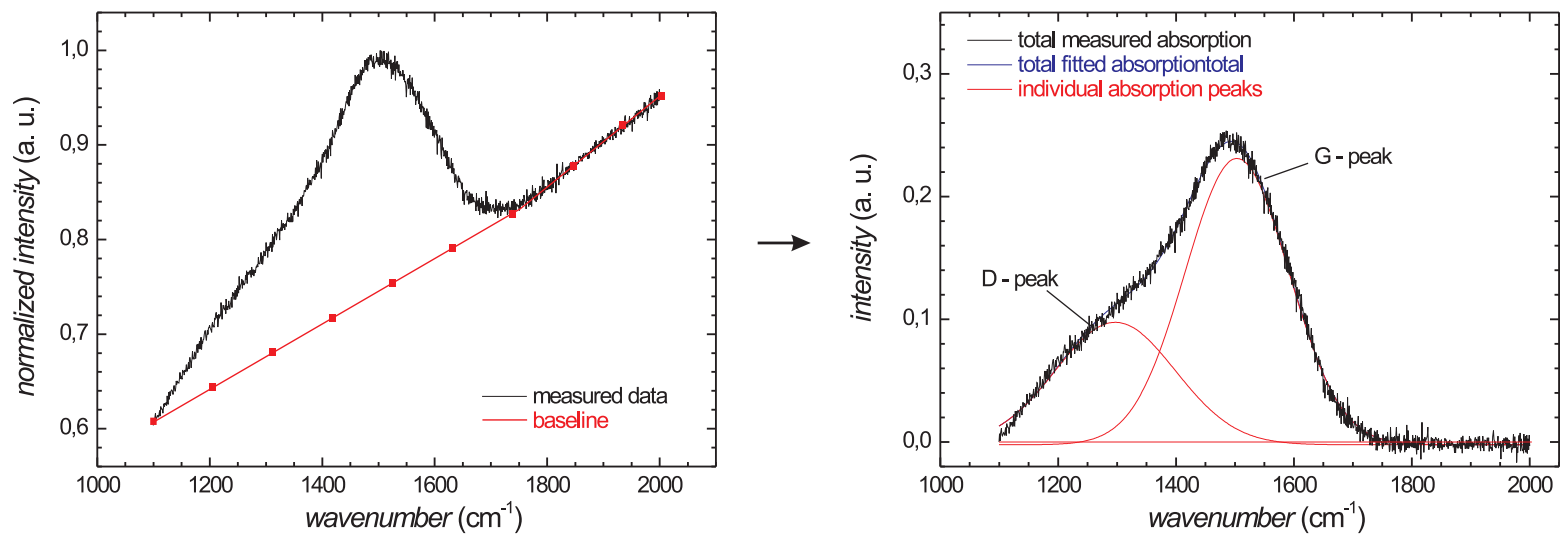

Figure 3.10: The baseline correction of the Raman spectra. Left graph: measured spectra and baseline; right graph: deconvoluted spectra.

tal amount of hydrogen can be underestimated. The second is the underestimation of the total amount of $s p^{3}$ content but overestimation of $s p^{2} / s p^{3}$ ratio since only carbon atoms bonded to at least one hydrogen atom are infrared active and the single bond between two carbon atoms is infrared inactive [4]. From this reason the interpretation of the infrared spectrum has to be done very carefully.

On a similar way the Raman spectra are treated. The measured spectrum (black line) and the position of the baseline (red line) are presented on the left graph in Fig. 3.10. The right graph in Fig. 3.10 shows the baseline corrected measured spectra (black line) and the deconvoluted one (two red peaks). The most prominent peaks in the Raman spectra of amorphous hydrogenated films are in general peaks around $1060 \mathrm{~cm}^{-1}$ detected only by UV excitation, $1360 \mathrm{~cm}^{-1}$ and $1560 \mathrm{~cm}^{-1}$ which can be detected by visible excitation [27]. The peaks are labeled as $T, D$ and $G$ peaks and the last two are designated on the right graph in Fig. 3.10. Another peak sometimes found in the Raman spectra of such films is located around $1450 \mathrm{~cm}^{-1}$. The origin of this peak is from a trans-polyacetylene phase in the film [28].

For the fitting the $D$ and $G$ peaks Lorentzians or Gaussians line shape are used. A Lorentzian fit is more often used in the case of disordered graphite while a Gaussian line shape is expected in disordered materials. Sometimes as an alternative for Gaussian fit a Bright-Wigner-Fano (BWF) line for $G$ peak in combination with a Lorentzian fit for the $D$ peak is used [24]. 


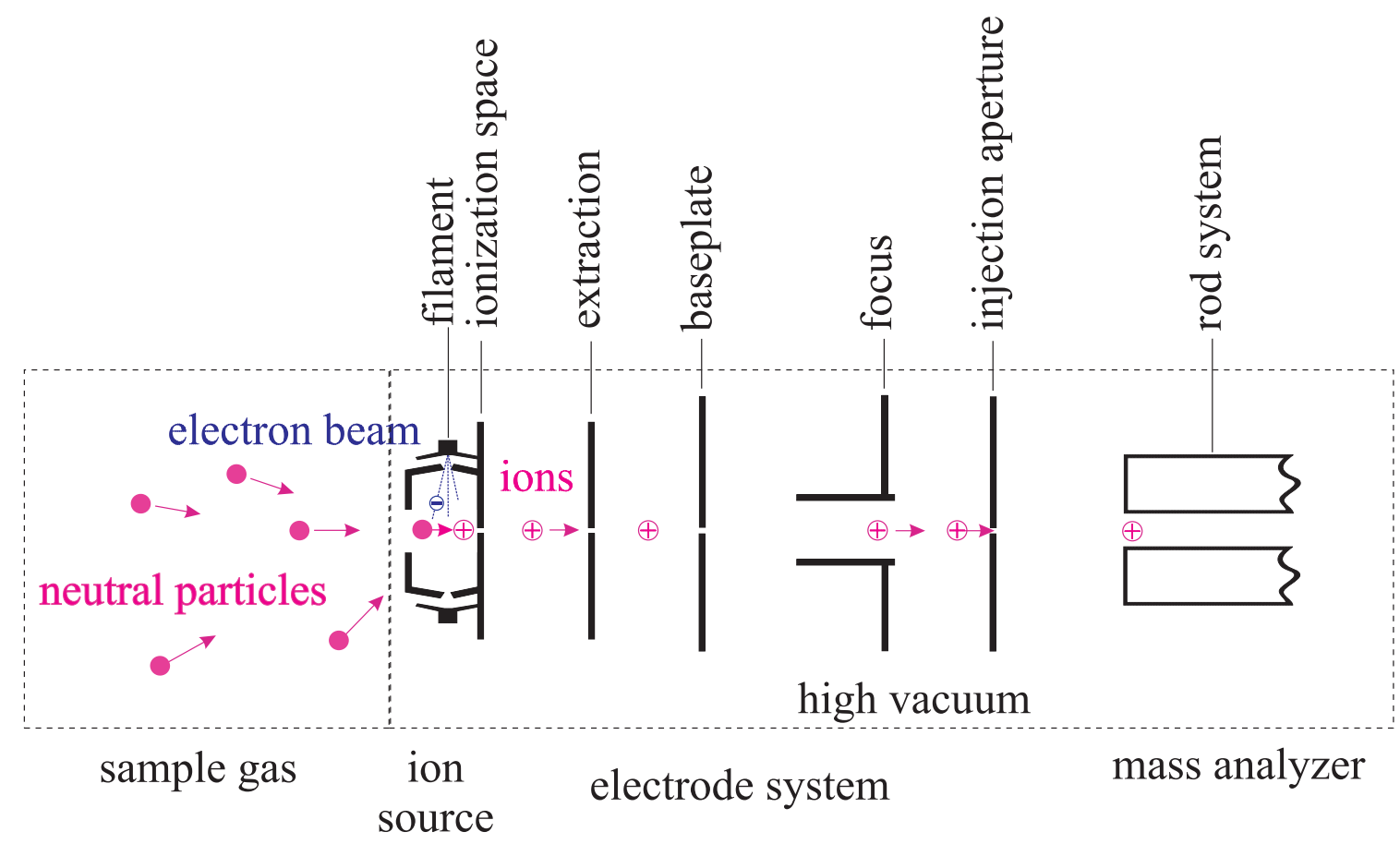

Figure 3.11: Scheme of the mass spectrometer.

\subsection{Mass spectrometry}

Mass spectrometry is an analytical technique used for identification of unknown compounds and for elucidation of the chemical composition of the sample. To obtain the mass spectrum, the individual components must be separated prior to masses. Samples in gas phase are preferred for mass analysis. If the sample is not in the gas phase (solid, liquid) it has to be transformed into the gas phase first and than it can be introduced in the source region. Mass spectrometry is very often used for the plasma monitoring during the plasma processing (deposition, etching). A sketch of the mass spectrometer is presented in Fig. 3.11.

Generally, the mass spectrometer consists of five parts: gas inlet system, ion source, mass analyzer, detector system for data controlling and acquisition system. A mixture of positive and negative ions and neutrals (molecules, radicals) enter the mass spectrometer through the extraction orifice. In order to be analyzed, molecules and radicals have to be first ionized. Electrons are emitted by the filament and accelerated in the ionization space (formation space). The electron ionization source (where positive ions are produced by electron impact) is the most common technique used for ionization of neutrals. After the ionization, ions are accelerated into the mass analyzer by an electric field. A schematic representation of the mass spectrometer is given in Fig. 3.11.

The measured intensity of mass $k$ for molecule $j$ is: 


$$
I_{j, m} \propto I_{e} \cdot N_{Q j} \cdot V_{Q j} \cdot \sigma_{j, m} \cdot \tau_{k}
$$

where $I_{e}$ is the electron current, $N_{Q j} \cdot V_{Q j}$ is the molecular flux in the ionization chamber, $\sigma_{j k}$ is the partial cross section and $t_{k}$ is the transmission in mass. The transmission in mass of the molecule/atom can be calculated with the relation:

$$
\tau_{k}=\frac{I_{k}}{\sigma_{k}}
$$

$I_{k}$ is the ion intensity of mass $k$ [29].

The analyzer separates the ions according to their mass-to-charge ratios $m / q$. One of the most commonly used analyzers is the quadrupole mass filter. The quadrupole mass analyzer consists of four parallel cylindrical rods made of steel in a square array. The ion motion is determined by a simultaneously applied constant direct current (DC) voltage $U$ and radio frequency (RF) voltage $V \cdot \cos \omega t$ on the electrodes. The appropriate combination of DC and RF voltages on the quadrupole rods enables passing only the ions of selected mass-to-charge ratio. All other ions have such a trajectory that disables their passing through the quadrupole mass analyzer and reaching further the detector.

From the detector the signal is sent to the system for monitoring and data acquisition. 


\section{Chapter 4}

\section{The role of hydrogen atoms in dust particle formation}

\subsection{Introduction}

In 1920 Langmuir and coworkers [5] described, for the first time, the formation of nano or dust particles in the reactive plasmas due to a agglomeration process in the plasma volume. Dust particles formation is observed during the different plasma processes used for etching [30], sputtering [31, 32] or thin film deposition [33]. In fabrication of integrated circuits particles created inside the plasma reactor can fall down onto the treated surface and cause severe damages of components. For that reason dust particles are named as "killer particles" in semiconductor industry. Even when they are so small that they are not able to cause any damage during the plasma processing of micro components they may be embedded into the film matrix changing the film properties and forming defects and voids at the interface with the surrounding film [34]. Sometimes the incorporation of the nanoparticles is wanted in plasma processing of the film because this is the only way to achieve desirable properties as for example in the case of photoluminescent thin films deposited in silane plasma with embedded crystalline clusters with sizes of 1 to $5 \mathrm{~nm}$ [35]. For these applications it is necessary to control the size [36] and the number of embedded nanoparticles [37, 38, 39].

Dust particles are found not only in industry and laboratory plasmas but also in space. Dust particles are found in diffuse and dense interstellar media [40, 41, 42], circumstellar shells [43], dark interiors of molecular clouds [44], nova ejecta [45], the outflow of red giant stars and accretion disks, proto-planetary nebula, interstellar medium of other galaxies $[46,47]$ etc. In spite of huge difference in spatial dimensions and temporal scales there are a lot of physical processes which are in common for processes occurring in the universe and the processes performed in the laboratory [48] allowing them to be investigated in laboratory conditions, in a more controllable 
environment.

The formation of particles in reactive plasmas is observed for various kinds of different precursor gases as, for example, in fluorocarbons [49, 50], silane [51, 52, $53,54,55]$ or in hydrocarbons $[56,57,58,59,60]$. In this work the focus is on the formation of particles in acetylene plasmas. Hydrocarbon plasmas in general are not only interesting for technical applications where they are used for the deposition of diamond or diamond-like films. They are also important for astrophysical investigations since they allow to produce so called astroanalogues i.e. materials that can be used for the interpretation of astronomical observations [48].

Particles in the plasma can originate from two processes - the homogeneous and the heterogeneous nucleation process. Particles formed in the homogeneous type of nucleation have a more or less regular spherical shape. They originate through the consecutive chemical reactions in the plasma volume where the precursor gas molecules are ionized and dissociated. The other kind of particles originates via a heterogeneous process are flake like. These flakes are larger and have irregular shape. They appear in the plasma due to the ion bombardment or reactive etching and sputtering of the film deposited onto the surfaces of the reactor. Particles can also be externally injected into the plasma. In this work only the particles originated in homogeneous nucleation process are investigated.

Reports on dust formation in hydrocarbon plasmas together with proposed models of nucleation were already published in the 1970s [61, 62]. Although lately published articles offer some insight into the mechanism of the growth processes and possible responsible precursors in hydrocarbon plasmas, the chemical picture of the nanoparticles formation in such plasmas is still not clear.

Acetylene as a monomer is chosen in this investigations because it is well known that $\mathrm{C}_{2} \mathrm{H}_{2}$ plays an important role in dust formation in hydrocarbon plasmas. Kobayashi et al [61] and Tibbitt et al [62] show that in saturated and unsaturated hydrocarbons plasma polymerization follows as soon as the acetylene compound is formed. Furthermore, acetylene undergoes a polymerization process instantaneously.

Via the dissociation process in any hydrocarbon gas, besides carbon carrying species, hydrogen in both forms, molecular and atomic, is formed as an important constituent of the discharge. It is identified by mass spectrometry [63, 64]. It remains in the discharge due the low pumping speed long enough to play an important role in the plasma reactions and, on that way, on the plasma chemistry. It also contributes significantly to the flux of radicals impinging onto the surface of the growing hydrocarbon film and on the surface of the growing particles.

Particles formed in the plasma change significantly plasma parameters such as 
electron density, electron temperature and, consequently, plasma composition [65, $66,67]$. All this, therefore, leads to changes in parameters which are relevant for the process, as for example, the self-bias, the matching of the power to the plasma, the sheath's width and voltage $[68,69]$ etc. The presence of particles also reduces the growth rate of the film.

In spite of all of these investigations, only little is known about the role hydrogen atoms play in the formation of dust particles in hydrocarbon discharges. The aim of this chapter is to elucidate the influence of hydrogen atoms on the formation process of dust particles and the suppression of particles formation in low pressure $\mathrm{C}_{2} \mathrm{H}_{2} \mathrm{RF}$ discharges.

The structure of this chapter is as following. Section 4.1 contains the introduction. In section 4.2 some general aspects of dust particle formation, as the steps in particles origin and forces acting on particles in the plasma, are given. Section 4.3 contains the results concerning the influence of hydrogen atoms on the dust particle formation leading, under some conditions, to the complete suppression of the particle formation. This is the content of section 4.3.1. The chemical composition of plasma and influence of hydrogen atoms on it is the content of 4.3 .2 section. At the end of this part in section 4.3.3 the short summary of observations and results is given.

\subsection{Some aspects of dust particle formation}

\subsubsection{Time development of particle formation and growth}

\section{Nucleation}

It is generally assumed that the particle formation starts with the creation of negative ions in the plasma. Ions are trapped in the plasma volume due to the confining plasma potential between the plasma and the plasma reactor wall. Being trapped in the plasma volume negative ions reside long enough to react with the precursor gas molecules and to create in consecutive chemical reactions macromolecules. Via monomer additions protoparticles nucleate and reaching a size $r_{p}$ of several $\mathrm{nm}$. In this formation phase protoparticles are either neutral or singly charged anions or cations due to the charge exchange.

\section{Coagulation}

During the next phase, called coagulation or agglomeration, protoparticles coalesce creating macroparticles with the diameter of about $50-60 \mathrm{~nm}$ in about $5 \mathrm{~s}$. This 


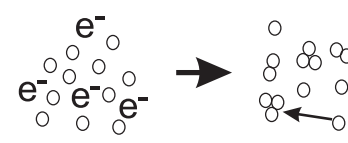

formation of primary clusters

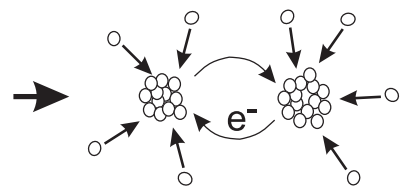

nucleation and cluster growth

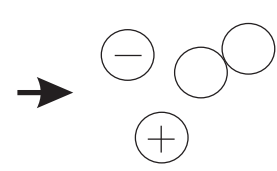

coagulation

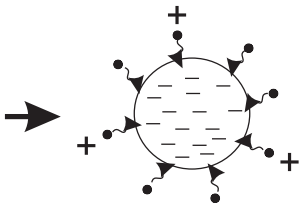

particle growth

Figure 4.1: Schematic presentation of the time development of dust particle formation and growth (after [70]).

process occurs rapidly, like a phase transition. Due to the coagulation the number of protoparticles decreases by several orders of magnitude while the particle volume fraction $4 / 3 \cdot \pi \cdot r_{p}^{3} \cdot N_{p}\left(r_{p}\right.$ - the particle radius, $N_{p}$ - the particle density) stays almost constant. The effective surface area is now much bigger so macroparticles collect large number of electrons and ions from the plasma bearing the net negative charge of tens to hundreds of electron charges. For that reason the concentration of electrons decreases giving a rise to the so-called $\alpha-\gamma^{\prime}$ transition phenomenon. Due to the lowering of the free electron concentration their energy increases to compensate for the electron losses by additional ionization.

\section{Surface growth}

The further growth of particles occurs by sticking of positive ions and neutral precursor molecules on the particles surface. Particles can reach micrometer sizes. After some time, the electrostatic force is no longer strong enough to keep the particles confined inside the plasma and other forces acting on the particles, above all the ion drag force, become important. In one moment forces are not any longer in equilibrium and particles are pushed out of the discharge.

These phases are schematically presented in Fig. 4.1.

\subsubsection{Charging of particles in plasmas}

Since the particles immersed in the plasma act as microprobes they collect charge. Because electrons, due to the much lower mass, have much higher velocities then ions, particles are negatively charged. Due to that particles interact with the electrons, ions and the other particles in the plasma as well as with the electromagnetic fields. On the other side, plasma properties are influenced by the particles presence. Because of these reasons, the particle charge is a very important plasma parameter. The particle charging can be expressed by the equation [71]: 


$$
\frac{d Q_{d}}{d t}=\sum_{i} I_{i}
$$

where $I_{i}$ are mainly the electron and ion currents on the particle, since other charging mechanisms in laboratory plasmas, as the secondary electron emission, can be neglected. These processes play a significant role in the process of cosmic dust particles charging.

The plasma response on the particle negative charging is the creation of a positive space charge around the particle. A measure of the shielding or the distance of screening of the electric field of a particle is the linearized Debye length:

$$
\frac{1}{\lambda_{D}^{2}}=\frac{1}{\lambda_{D_{e}}^{2}}+\frac{1}{\lambda_{D_{i}}^{2}}
$$

where

$$
\lambda_{D_{e(i)}}=\left(\frac{\epsilon_{0} \cdot k_{B} \cdot T_{e(i)}}{e^{2} \cdot n_{e(i)}}\right)^{\frac{1}{2}},
$$

are the electron and ion Debye lengths, $\epsilon_{0}$ is the permittivity of vacuum, $k_{B}$ is the Boltzmann constant, $T_{e(i)}$ is the electron (ion) temperature, $n_{e(i)}$ is the electron (ion) density, respectively, and $e$ is the elementary charge. The value of the electron Debye length in a capacitive coupled plasma with electron density $n_{e}=10^{8} \mathrm{~cm}^{-1}$ and electron temperature $T_{e}=1 \mathrm{eV}$ is $\lambda=0.07 \mathrm{~cm}$. In an inductively coupled plasma for the same electron temperature and typical value of electron density $n_{e}=10^{11} \mathrm{~cm}^{-1}$ the electron Debye length has a value of $\lambda=25 \mu \mathrm{m}$.

After the electron and ion fluxes toward the particle surface are balanced, in the stationary state, the particle has a negative floating potential determined by the electron temperature:

$$
\varphi \sim-\frac{T_{e}}{Z e}
$$

where $Z e$ is the charge acquired by particle immersed in the plasma. For example, the particles of diameter $1-10 \mu \mathrm{m}$ acquire a negative charge of $10^{3}-10^{4}$ electrons. The potential on the particle surface is of the order $T_{e}$ to $2 T_{e}$ (see Ref. [72] and references therein).

The time evolution of the particle charge can be expressed by the equation:

$$
\frac{d Q_{d}}{d t}=I_{i}-I_{e}=0
$$

meaning that, in the stationary state the ion $\left(I_{i}\right)$ and electron $\left(I_{e}\right)$ fluxes are balanced and using the orbital motion limited approximation these fluxes can be calculated. 
In this approximation it is assumed that the sheath around the particle is collisionless since the electron-neutral and the ion-neutral mean free paths are much larger then the sheath width. Depending on the distance from the particle (the impact parameter), the ion moving in the central filed of the negatively charged particle can be attracted by the particle (collected) or its trajectory can be changed (deflected). The maximum distance from the particle when the ion can still be attracted by the particle is called the critical impact parameter, $b_{\text {coll }}$. This critical distance can be calculated from the conservation of energy and the angular momentum at that distance and the expression for the critical impact parameter is:

$$
b_{\text {coll }}^{2}=r_{p}^{2} \cdot\left(1-\frac{2 \cdot e \cdot \varphi}{m_{i} \cdot v_{i}^{2}}\right) .
$$

where $m_{i}$ is the ion mass and $v_{i}$ is the ion initial velocity, $r_{p}$ the particle radius and $\varphi$ is the particle potential. Thus, all ions approaching on distances smaller then $b_{\text {coll }}$ are collected while the ions approaching with the impact parameter bigger then $b_{\text {coll }}$ are deflected. The expression for the electron flux has the form (for details see [70] and references therein):

$$
I_{e}=\pi \cdot r_{p}^{2} \cdot e \cdot n_{e} \cdot \sqrt{\frac{8 \cdot k_{B} \cdot T_{e}}{\pi \cdot m_{e}}} \cdot \exp \left(\frac{e \cdot \varphi}{k_{B} \cdot T_{e}}\right)
$$

and similar to that for the ion flux:

$$
I_{i}=\pi \cdot r_{p}^{2} \cdot e \cdot n_{i} \cdot \sqrt{\frac{8 \cdot k_{B} \cdot T_{i}}{\pi \cdot m_{i}}} \cdot\left(1-\frac{e \cdot \varphi}{k_{B} \cdot T_{e}}\right) .
$$

where $v_{t h, e(i)}=\sqrt{8 \cdot k_{B} \cdot T_{e(i)} / \pi \cdot m_{e(i)}}$ is the electron (ion) thermal velocity.

\subsubsection{Forces acting on particles in plasmas}

Dust particles suspended in the plasma are subject to various forces which determine their motion and confinement in the plasma volume. The forces acting on dust particles can be divided into two groups: forces with electric nature (electron drag, ion drag and electrostatic), and forces independent of charge (gravity, neutral drag and thermophoresis).

\section{Ion drag force}

The ion drag force appears in the momentum transfer from the ions to particles embedded into a plasma. It can play a very important role when the ion flux is large, for sufficiently high powers. The ion drag force consists of two components and the total ion drag force is: 


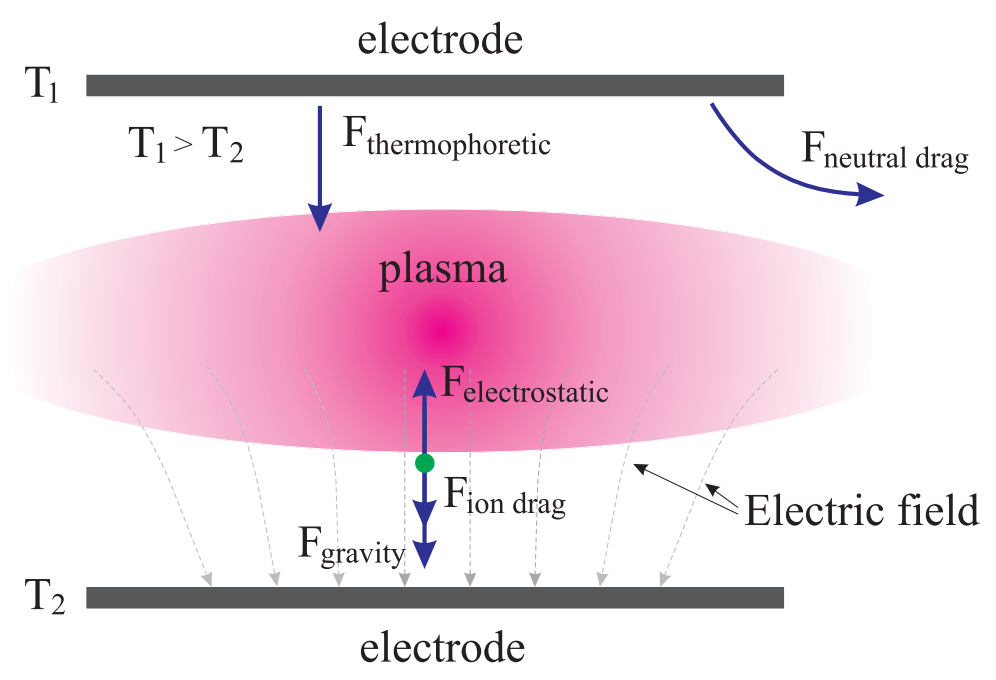

Figure 4.2: Forces acting on dust particles in the plasma.

$$
\overrightarrow{F_{i d}}=\overrightarrow{F_{i d}^{c}}+\overrightarrow{F_{i d}^{o}}
$$

where $\overrightarrow{F_{i d}^{c}}$ is the collection force and results from the momentum transfer when a positive ion is collected by the particle and has the form [73]:

$$
\overrightarrow{F_{i d}^{c}}=\pi \cdot b_{c o l l}^{2} \cdot n_{i} \cdot v_{s} \cdot m_{i} \cdot \overrightarrow{u_{i}}
$$

where $b_{\text {coll }}$ is the collection impact parameter given by equation 4.6 and $v_{s}$ is the mean velocity of the ions. The second component, $\overrightarrow{F_{i d}^{o}}$, is the orbit force and is given by:

$$
\overrightarrow{F_{i d}^{o}}=4 \pi \cdot b_{\pi / 2}^{2} \cdot \Gamma \cdot n_{i} \cdot v_{s} \cdot m_{i} \cdot \overrightarrow{u_{i}},
$$

where $b_{\pi / 2}$ is the impact parameter at $90^{\circ}$ angle deflection and $\Gamma$ is the Coulomb logarithm

$$
\Gamma=\frac{1}{2} \cdot \ln \left(\frac{\lambda_{D}^{2}+b_{\pi / 2}^{2}}{b_{\text {coll }}^{2}+b_{\pi / 2}^{2}}\right) .
$$

The incoming positive ion, deflected by the potential around the particle, transfers its momentum during the electrostatic Coulomb interaction to the particle. The ion drag force always pushes the particles from the ionization zone.

\section{Electrostatic force}

The electrostatic force is the force which a dust particle experiences in the plasma when a non-zero macroscopic field is applied. Daugherty, Porteous and Graves [74] 
and Hamaguchi and Farouki [75] showed that this force can be well approximated by the vacuum force:

$$
\overrightarrow{F_{e s}}=Q_{d} \cdot \vec{E},
$$

if the plasma can be considered as uniform, where $Q_{d}$ is the charge on the dust particle and $\vec{E}$ is the applied electric field.

\section{Electron drag force}

As the ion drag force, the electron drag force appears due to the momentum transfer from the electrons to the charged particles. In the binary collision approximation, the magnitude of the electron drag force, $\overrightarrow{F_{e d}}$, is given by:

$$
\vec{F}_{e d}=m_{d} \cdot \nu_{d e} \cdot \overrightarrow{u_{e}},
$$

where $u_{e}$ is the relative drift velocity between the electrons and the dust particles, and $\nu_{d e}$ is the momentum exchange rate with the background medium through particle-electron collisions given by equation:

$$
\nu_{d e} \approx(2 \sqrt{2 \pi} / 3) \cdot\left(m_{e} / m_{d}\right) \cdot n_{e} \cdot v_{t h, e} \cdot r_{p}^{2} \cdot z^{2} \cdot \Lambda_{d e},
$$

where $n_{e}, m_{e}$ and $v_{t h, e}$ are density, mass and thermal velocity of electrons, $r_{p}$ is the particle radius, $z$ :

$$
z=\frac{|Z| \cdot e^{2}}{r_{p} \cdot T_{e}}
$$

is the absolute magnitude of the particle charge $e Z$ in units of $r_{p} \cdot T_{e} / e^{2}$ and $\Lambda_{d e}$ is the Coulomb logarithm for particle-electron collisions. For details see for example [76] and references therein.

This force is very weak due to the very small value of electron-to-ion mass ratio so the effect of this force is usually negligible in comparison with the effects of other forces. This is true in discharges where the drift velocities are comparable, i.e. $u_{e} \sim u_{i}$ as, for example, in RF discharges. But in discharges where $u_{e} \gg u_{i}$ this is not quite obvious. This is the case in weakly ionized plasma (e.g. the positive column of a DC gas discharge) where the force ratio is:

$$
F_{i} / F_{e} \sim\left(T_{e} / T_{i}\right)^{2} \cdot\left(\sigma_{e n} / \sigma_{i n}\right),
$$

where $\sigma_{e n}$ and $\sigma_{i n}$ are the effective momentum transfer cross sections for electron neutral and ion - neutral collisions [77]. 


\section{Neutral drag force}

The momentum transfer from the neutral gas atoms and molecules, which the dust particle exhibits during the collisions when moving through the gas, results in the neutral drag force. A regime of the estimation of the neutral drag force is determined with the value of the Knudsen number, $K_{n}$. The Knudsen number, defined as the ratio of the mean free path of the neutral gas molecules to the dust particle radius, $K_{n}=\lambda_{n} / r_{d}$, determines two regimes: the hydrodynamic for low values of the Knudsen's number (high pressure) and the kinetic or 'long free path' regime for large values of the Knudsen's number (this is the case in typical low-pressure discharges with $p<1$ Torr). In the first case of small Knudsen's numbers, $K_{n} \gg 1$, the Stokes expression:

$$
F_{n d}=-6 \cdot \pi \cdot \eta \cdot r_{d} \cdot v_{d}
$$

can be applied, where $\eta$ is the velocity of the neutral gas and $v_{d}$ is the relative velocity between particle and gas.

In the kinetic regime, i.e. in the case of large Knudsen's number, $K_{n} \gg 1$, the neutral drag force has the form [78]:

$$
\vec{F}_{n}=-\frac{4}{3} \pi \cdot r_{d}^{2} \cdot m_{n} \cdot n_{n} \cdot v_{t h, n}\left(\vec{v}_{d}-\vec{v}_{n}\right),
$$

where $m_{n}$ is the mass of the gas, $n_{n}$ is the neutral gas density, and $v_{t h, n}$ is the average thermal velocity of the gas defined as $v_{t h, n}=\sqrt{8 \cdot k_{B} \cdot T / \pi \cdot m_{n}}$. The minus sign designates the opposite direction of the force to the relative velocity.

\section{Thermophoresis}

If one of the electrodes is heated or cooled the temperature in a gas is not uniform, therefore a temperature gradient will appear. In the hotter region gas molecules will have higher velocity then in the colder region and the net momentum transfer rate from the gas molecules to the dust particle is larger on the hotter side of the particle than on the particle's colder side. The thermophoretic force is induced due to this asymmetry in the net momentum transfer. It acts in the direction of the temperature gradient $-\nabla T_{n}$, pushing the particles toward the colder region. The analytical expression for this force is derived by Talbot et al. [79] for gas temperatures less than $500 \mathrm{~K}$ :

$$
\vec{F}_{t h}=-\frac{4 \sqrt{2 \pi}}{15} \frac{r_{d}^{2}}{v_{t h, n}} \cdot k_{T} \cdot \nabla T_{n}
$$



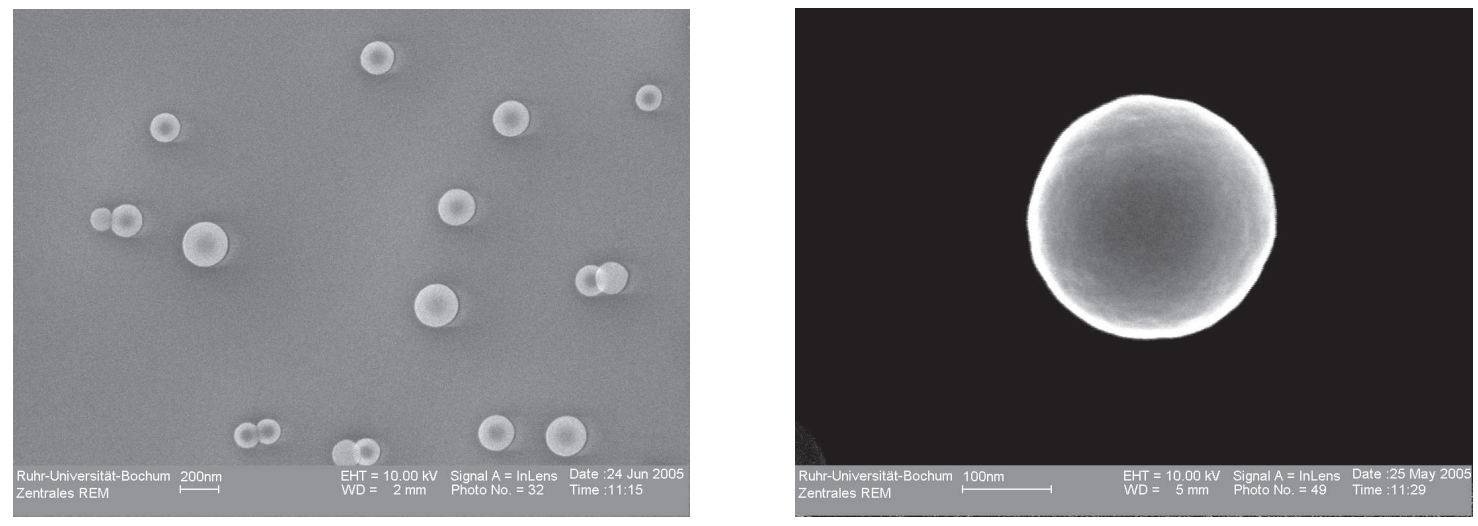

Figure 4.3: SEM images of dust particles produced in an inductively coupled acetylene plasma running in the capacitive or so-called E-mode. Left: a few particles collected in the acetylene plasma on the surface of the wafer. The particles have a broad size distribution. Right: a typical plasma-generated dust particle. All ICP particles generated in the acetylene discharge have a very smooth surface.

where $v_{t h, n}$ is the gas thermal velocity, $k_{n}$ is the translation thermal conductivity coefficient of the gas, and $T_{n}$ is the gas temperature. The thermophoretic force does not depend on gas pressure and temperature.

\section{Gravitational force}

The gravitationalforce is proportional to the mass of the dust particle, i.e. to the particle's density and volume:

$$
\vec{F}_{g}=m_{d} \cdot \vec{g}=4 / 3 \cdot \pi \cdot r_{d}^{3} \cdot \rho \cdot \vec{g}
$$

where $g$ is the gravitational acceleration, $r_{d}$ is the dust particle radius. Under the influence of this force particles will move in the direction of the lower electrode. Since it scales with $a^{3}$ it will become dominant only for large particles.

\subsection{Results}

The effect of hydrogen atoms on the formation of dust particles in the early stage of their formation is studied in an experiment performed with a gas mixture of $10 \mathrm{sccm}$ acetylene and $10 \mathrm{sccm}$ molecular hydrogen. The working pressure in the chamber is 0.06 mbar and during the experiment it does not change due to the polymerization in the plasma volume. The power applied to the coil is $75 \mathrm{~W}$. Under these conditions the discharge operates in the capacitive mode. The acetylene and the hydrogen 
molecules are injected into the chamber on the way described in detail in chapter 2 . During the experiment the lower electrode is cooled.

An analysis performed by the secondary electron microscope shows that dusty particles produced in an inductively coupled acetylene discharge running in the capacitive mode have a broad size distribution. Their size ranges from about 150 $\mathrm{nm}$ up to $500 \mathrm{~nm}$. Photos of hydrocarbon dust particles (left) and one dust particle (right) collected in the acetylene discharge are presented in Fig. 4.3. It can also be observed that the hydrocarbon dust particles from this discharge have a very smooth surface. The growth speed of particles in this discharge is extremely high. All this contrasts to the case of dust particles created in a capacitively coupled discharge where particles have very narrow size distribution [80, 81] cauliflower shape and very low growth speed $[80,81,82]$ of approximately $10 \mathrm{~nm} / \mathrm{min}$. The reason for this very high growth speed in our experiment is that we work with the pure acetylene which flow rate is the ten times higher then in experiments preformed in $[80,81,82]$, where a mixture of argon and acetylene is used.

\subsubsection{Influence of hydrogen atoms on dust particle formation}

The measurements of the intensity of the scattered laser light reveal that the acetylene plasma spontaneously produces dust particles during the film deposition process. The dust particles start forming soon after the discharge is ignited. The formation process of dust particles is monitored by illuminating a dust cloud with a He-Ne $(5 \mathrm{~mW})$ laser light and by measuring the intensity of the laser light scattered by the particles at $90^{\circ}$. It should be noted, that this yields a measurement along the laser beam, not an average over the particle content in the whole reactor.

The time behavior of the scattered laser light measured in the center of the discharge is presented in Fig. 4.4. The intensity of the scattered laser light exhibits a periodical behavior that is already known from previous works [83, 84]. Approximately $35 \mathrm{~s}$ after the ignition of the acetylene discharge the whole region between the electrodes is filled with dust particles and the intensity of the scattered light exhibits a maximum. A few seconds later a void (dust free region) develops in the center of the discharge and the intensity of the laser light rapidly decreases [85]. Then the void disappears and the whole process repeats itself.

The periodicity in the dust formation can be explained qualitatively by means of the interplay between the dust particle formation and the forces acting on them at the same time. The resultant force acting on the negatively charged particles is the 


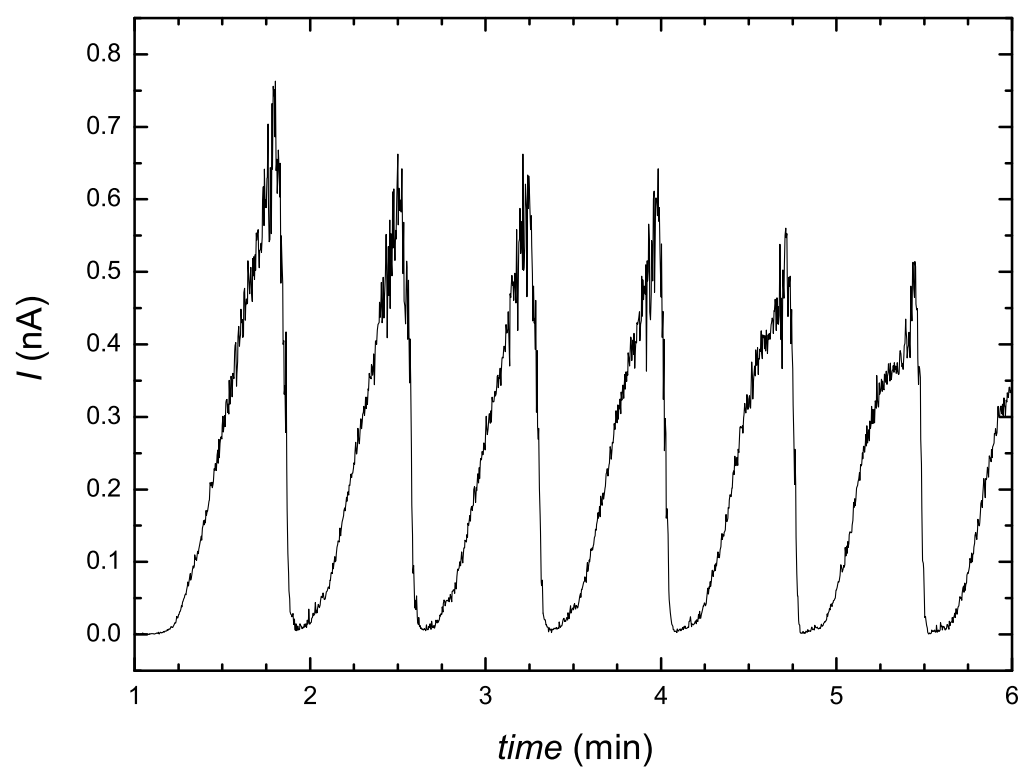

Figure 4.4: The intensity of the scattered laser light coming from the center of the discharge exhibits a periodic behavior due to dust formation.

sum of the different forces which scale with different powers of the particle radius $r_{p}$. At the early stage of the growth process the electrostatic force is strong enough to confine the particles and trap them between the electrodes. As the growth process continues the particle radius is increasing and the other forces become more and more important. Consequently, the particles are growing up to the moment when the forces acting on the particles stop being in the equilibrium. At that moment particles are pushed out of the discharge. After that a new growth cycle starts. The tree different stages in the dust particle formation and the void propagation are presented in Fig. 4.5. In the left photo the interelectrode space is completely filled with dust particles. This corresponds to the situation when the intensity of the scattered laser light has a maximum value. The middle photo shows how a void forms starting from the center of the discharge and expands aside slightly pushing the particles out of the discharge. During this phase, the intensity of the scattered laser light decreases. The right photo presents an almost completely developed void a few seconds before its disappearance. Only a small number of the particles is in the region between the electrodes and they are at the edge of the void. This moment is close to the minimum of the intensity of the scattered laser light. During this experiment the second discharge is not ignited, therefore, only the molecular 

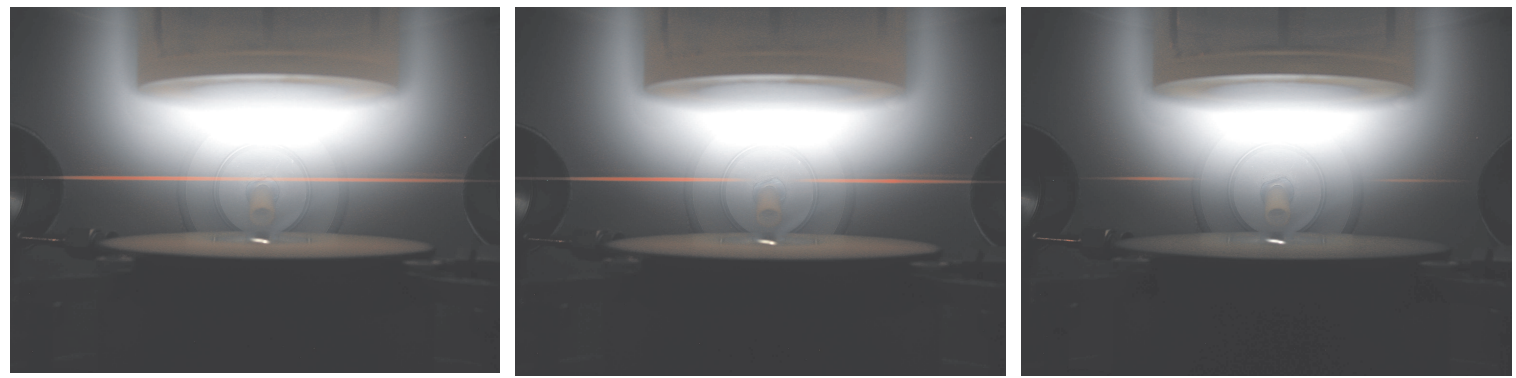

Figure 4.5: Photos of three different stages in the dust particles formation and the dust free region (void) development and its propagation. Left: the whole interelectrode region is filled with dust particles so the scattered laser light has homogeneous intensity along the horizontal direction of the discharge. Middle: the beginning of the dust free region formation in the center of the discharge causes a slight dust particle pushing and tearing of the line of the scattered laser light in the middle. Right: the further dust free region propagation and the pushing of the particles results in significant lost of the intensity of the scattered laser light.

hydrogen flows into the acetylene discharge.

Fig. 4.6 presents the time variation of the scattered laser light intensity and the effect of hydrogen atoms from the second discharge on the acetylene plasma. The variation of the amplitude of the scattered laser light intensity during the time indicates that the discharge needs some time after the ignition to reach a stable chemical composition. After the ignition of the second discharge at $t=8 \mathrm{~min}$ and the injection of hydrogen atoms into the acetylene discharge dust particles continued to grow until the end of the growth cycle. From this moment on particle formation is no longer detectable. When the second discharge is switched off the dust particle formation starts again spontaneously.

From this experiment it is obvious that as soon as the hydrogen atoms are injected into the acetylene discharge the further dust particle formation is completely suppressed. For the clarification of this situation the chemical composition of the plasma is monitored by mass spectroscopy.

\subsubsection{Mass spectra and hydrogen dilution}

\section{Time evolution of positive ions}

A positive ion mass spectrum in the acetylene discharge with addition of hydrogen molecules, measured by means of the plasma process monitor, is presented in Fig. 4.7. The presence of the groups with even number of hydrocarbon ions, $\mathrm{C}_{2 m} \mathrm{H}_{n}^{+},(m, n=1,2, \ldots)$ is prominent being characteristic for acetylene [57]. In the 


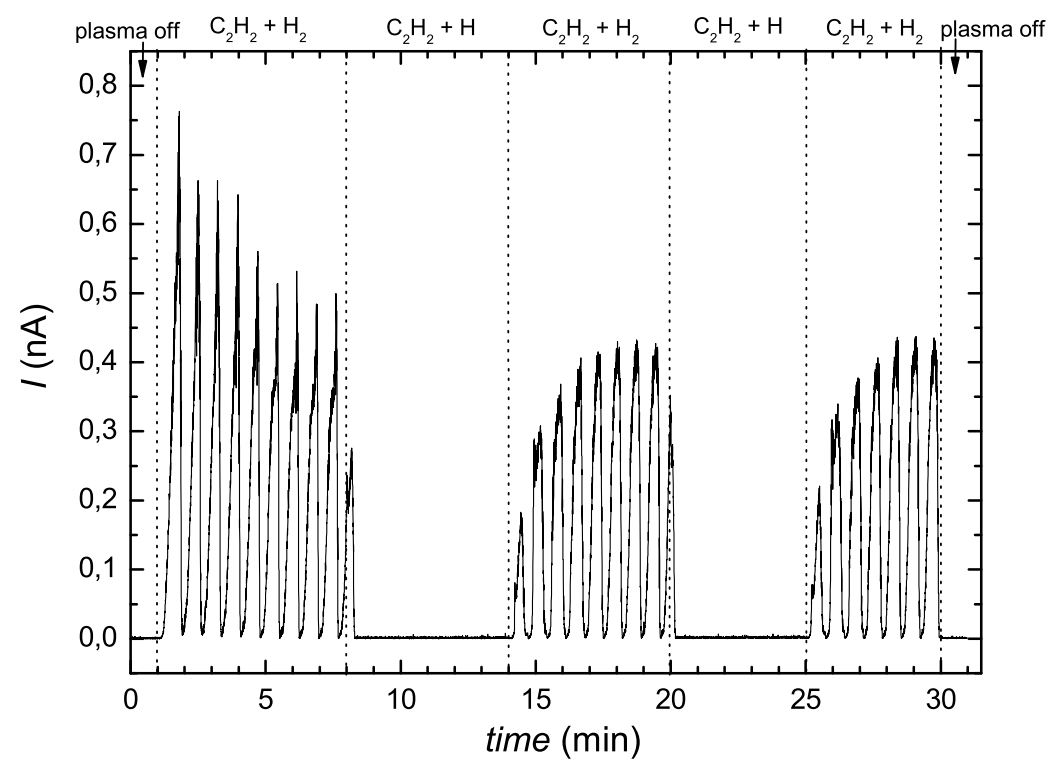

Figure 4.6: Temporal behavior of the dust particle formation in the $\mathrm{C}_{2} \mathrm{H}_{2}+\mathrm{H}_{2}$-plasma and the complete suppression of the dust particle formation after the addition of hydrogen atoms.

electron impact ionization process mainly $\mathrm{C}_{2} \mathrm{H}_{2}^{+}$ions are created. This is due to the lower threshold energy for the ionization compared with the threshold energy for the dissociative ionization processes. The threshold energy for the creation of $\mathrm{C}_{2} \mathrm{H}_{2}^{+}$ in an electron impact ionization process:

$$
\mathrm{C}_{2} \mathrm{H}_{2}+\mathrm{e}^{-} \rightarrow \mathrm{C}_{2} \mathrm{H}_{2}^{+}+2 \mathrm{e}^{-}
$$

is $11.4 \mathrm{eV}$ [86] while for the creation of $\mathrm{C}_{2} \mathrm{H}^{+}$in the dissociation ionization process:

$$
\mathrm{C}_{2} \mathrm{H}_{2}+\mathrm{e}^{-} \rightarrow \mathrm{C}_{2} \mathrm{H}^{+}+\mathrm{H}+2 \mathrm{e}^{-},
$$

it is $16.5 \mathrm{eV}$ [86]. A possible pathway in the creation of higher hydrocarbon ions can be:

$$
\begin{gathered}
\mathrm{C}_{2} \mathrm{H}_{2}^{+}+\mathrm{C}_{2} \mathrm{H}_{2} \rightarrow \mathrm{C}_{4} \mathrm{H}_{2}^{+}+\mathrm{H}_{2}, \\
\mathrm{C}_{4} \mathrm{H}_{2}^{+}+\mathrm{C}_{2} \mathrm{H}_{2} \rightarrow \mathrm{C}_{6} \mathrm{H}_{2}^{+}+\mathrm{H}_{2}, \\
\mathrm{C}_{6} \mathrm{H}_{2}^{+}+\mathrm{C}_{2} \mathrm{H}_{2} \rightarrow \mathrm{C}_{8} \mathrm{H}_{2}^{+}+\mathrm{H}_{2}, \ldots
\end{gathered}
$$




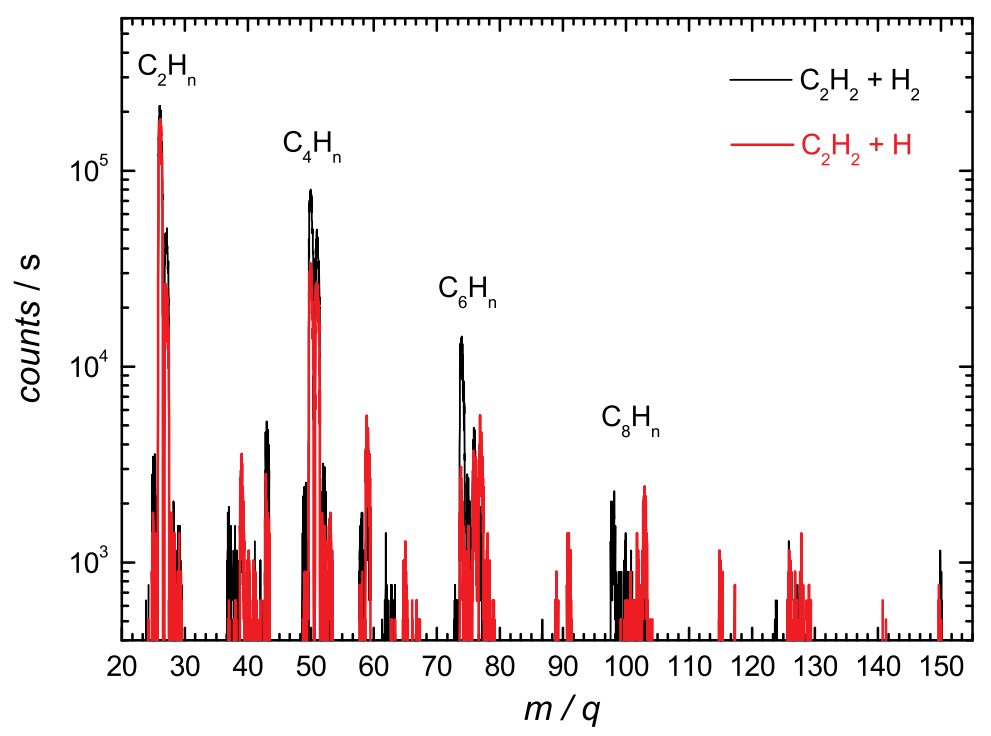

Figure 4.7: The positive ion mass spectrum. The black spectrum is measured with the hydrogen discharge switched off, the red spectrum is measured under the same discharge operation conditions but with the hydrogen discharge switched on.

Around these hydrocarbons there are the most prominent groups of masses in the ion mass spectra - $26\left(\mathrm{C}_{2} \mathrm{H}_{\mathrm{n}}\right), 50\left(\mathrm{C}_{4} \mathrm{H}_{\mathrm{n}}\right), 74\left(\mathrm{C}_{6} \mathrm{H}_{\mathrm{n}}\right), \ldots$

Except the groups around the even hydrocarbon molecules, a few groups with odd number of hydrocarbons can be observed too. They have a few orders of magnitude lower count rates due to the much higher threshold energy for dissociative ionization of acetylene molecules. The threshold energies for the electron impact dissociative ionization reactions:

$$
\begin{gathered}
\mathrm{C}_{2} \mathrm{H}_{2}+\mathrm{e}^{-} \rightarrow \mathrm{CH}^{+}+\mathrm{CH}+2 \mathrm{e}^{-}, \\
\mathrm{C}_{2} \mathrm{H}_{2}+\mathrm{e}^{-} \rightarrow \mathrm{C}^{+}+\mathrm{CH}_{2}+2 \mathrm{e}^{-},
\end{gathered}
$$

are $20.6 \mathrm{eV}$ and $20.3 \mathrm{eV}$, respectively [86]. Comparing these values with the threshold energy for $\mathrm{C}_{2} \mathrm{H}_{2}^{+}$ion creation in reaction (4.22) it is clear that the odd hydrocarbon ions are created in much smaller amount leading to less production of hydrocarbon ions with odd number of carbon atoms. The black spectrum in Fig. 4.7 comes from the acetylene discharge with the hydrogen discharge switched off, and the red spectrum is measured with the hydrogen discharge switched on. The decrease of the count rates for most of the masses after the injection of hydrogen atoms is noticeable. 


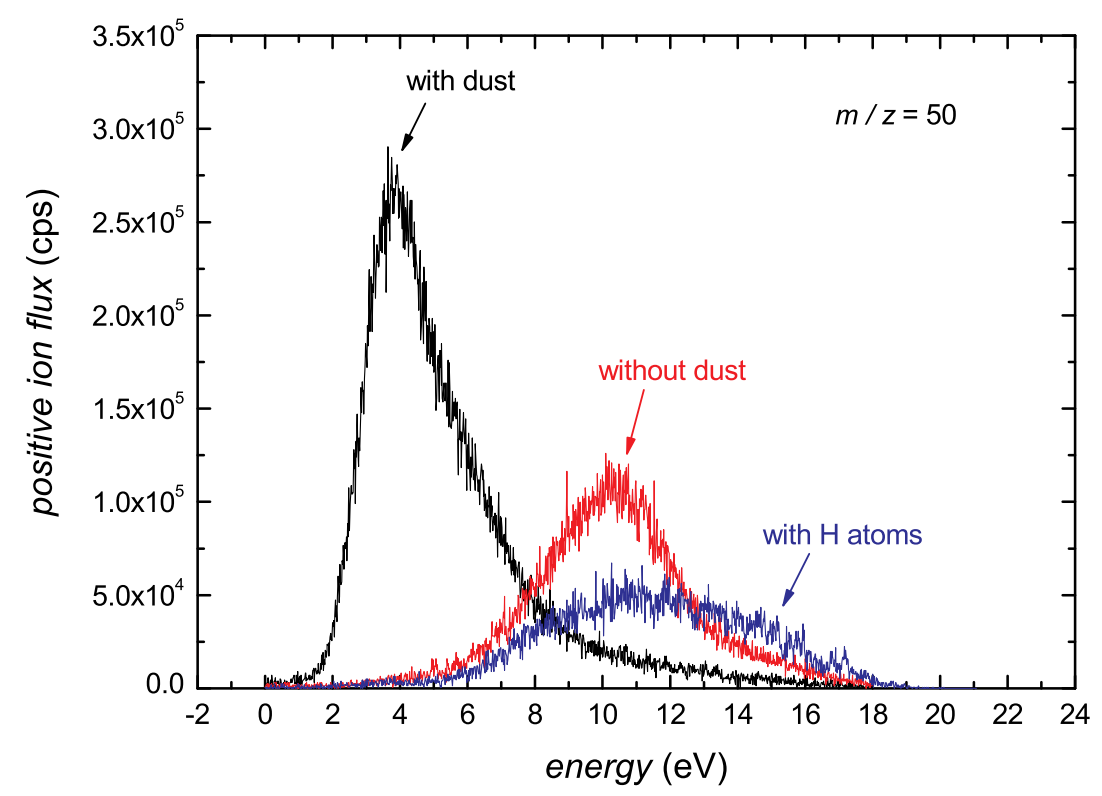

Figure 4.8: The positive ion energy distributions for the $m / q=50$ ion at different moments during dust particle formation. The black curve corresponds to the case when the molecular hydrogen is introduced into the acetylene discharge and to the moment when the whole chamber is filled with dust particles. The red curve is measured after the void is developed and the dust particles are pushed out from the discharge. The blue curve is obtained when the hydrogen atoms are injected into the acetylene discharge and the further dust particle formation is suppressed.

The explanation for this phenomenon is not simple because of at least two reasons. The plasma process monitor, in fact, does not measure the concentration of positive ions but the positive ion flux for a fixed ion energy. A second reason is that not only the addition of hydrogen atoms, but also the presence of dust particles changes the plasma parameters (electron density, electron temperature) and the sheath properties. This is clearly demonstrated in Fig. 4.8 where the positive ion energy distribution for $m / q=50$ at different moments in the dust particle formation is presented. The black curve represents the positive ion energy distribution in the case when the hydrogen molecules are introduced in the acetylene discharge and at the moment when the whole interelectrode region is filled with particles. In Figs. 4.4 and 4.6 that is the moment when the intensity of the laser scattered light exhibits a maximum. The red curve is the positive ion energy distribution under the same conditions but after the void development - when the interelectrode region is dust free. In Figs. 4.4 and 4.6 this situation corresponds to the minimum in the inten- 


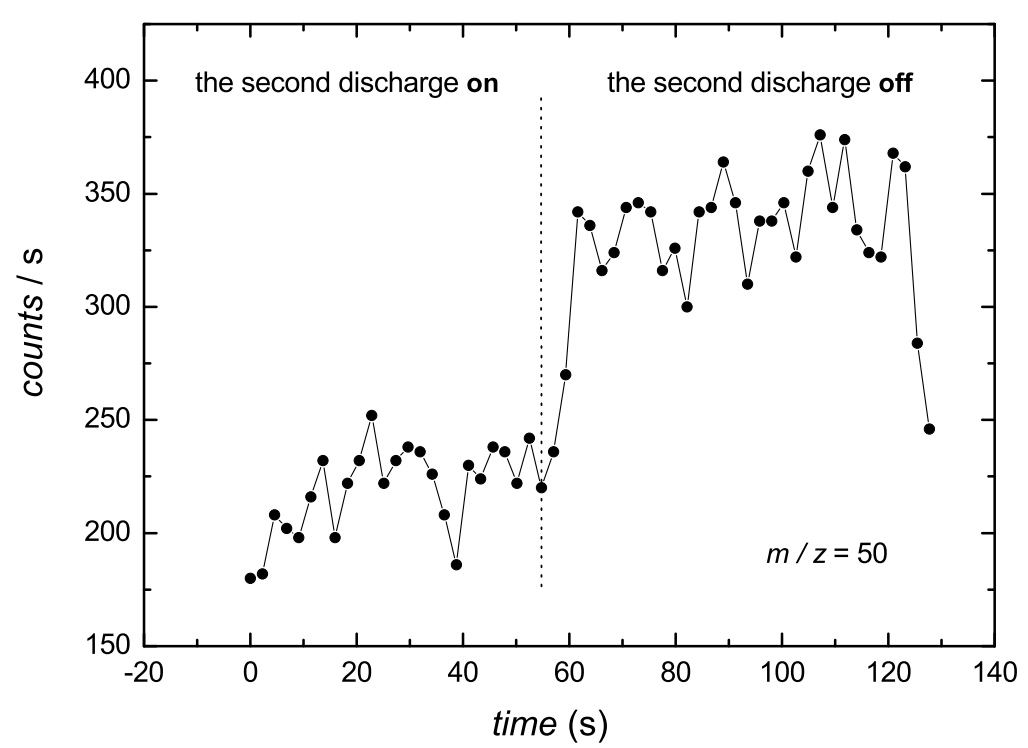

Figure 4.9: The change of the count rates of ion $\mathrm{C}_{4} \mathrm{H}_{2}^{+}(m / q=50)$ under the influence of hydrogen atoms and molecules. In the first phase the hydrogen discharge is switched on and the hydrogen atoms are added into the acetylene discharge. After about $60 \mathrm{~s}$ the hydrogen discharge is switched off and the hydrogen molecules are added into the acetylene discharge.

sity of the scattered laser light. The blue curve represents the positive ion energy distribution when the second discharge is switched on and the hydrogen atoms are injected into the acetylene discharge while all other discharge operation conditions were retained. This situation is the same as $\mathrm{C}_{2} \mathrm{H}_{2}+\mathrm{H}$ in Fig. 4.6 when further dust particle formation is suppressed by the hydrogen atoms. From this graph it is clear that the dust particles change the flux of the positive ions reaching the entrance of the mass spectrometer. Since the flux is the product of the number of particles and their velocity, the differences in positive ion energy distribution can appear from the reduced number of ions (consumption by dust particle growth) and/or from the change in their velocity due to the breakdown of the sheath.

To avoid this problem the acetylene flow is decreased to a value of $1 \mathrm{sccm}$ (all other discharge operation conditions are kept the same). No dust particles are observed under such conditions. Nevertheless, a similar phenomenon is observed. When the hydrogen discharge is switched on the intensity of the peak at ion $\mathrm{m} / \mathrm{q}$ $=50$ decreases in intensity compared to the situation when the second discharge is switched off. This observation is presented in Fig. 4.9. 
This clearly shows that the addition of hydrogen atoms prevents the formation of high-mass dust precursor molecules.

\section{Time evolution of neutrals}

The neutral mass spectrum, presented in Fig. 4.10, shows the same tendency for grouping as the positive ions do. The three obvious groups around the masses with even number of carbon atoms can be distinguished: acetylene (the precursor gas molecules) around the mass 26, biacetylene around mass 50 and triacetylene around mass 74 . The triple bond between the two carbon atoms in the acetylene molecule $(\mathrm{H}-\mathrm{C} \equiv \mathrm{C}-\mathrm{H})$ is much stronger $(\mathrm{C} \equiv \mathrm{C}$ is $835 \mathrm{~kJ} / \mathrm{mol})$ than the single $\mathrm{H}-\mathrm{C}$ bond $(436 \mathrm{~kJ} / \mathrm{mol})$. The acetylene molecule will rather lose one $\mathrm{H}$ atom in the electron induced dissociation process becoming ethynyl radical $\mathrm{C}_{2} \mathrm{H}$. This radical can then undergo condensation and can react with the $\mathrm{C}_{2} \mathrm{H}_{2}$ molecule followed by the release of one hydrogen atom. In this reaction $\mathrm{C}_{4} \mathrm{H}_{2}$ (biacetylene) is created. On the same way higher unsaturated polyacetylene radicals can be formed explaining the appearance of sequences of masses. The black spectrum in Fig. 4.10 presents the neutral mass spectrum measured when the second discharge is switched off and the hydrogen atoms are injected into the acetylene discharge, while the red spectrum is measured after the ignition of the second discharge and the injection of the hydrogen atoms under the same discharge operation conditions. These graphs show the change of the intensity of the masses after the injection of the hydrogen atoms into the acetylene discharge.

The time evolution of the neutral mass spectra can be seen from the graphs in Fig. 4.11 where the behavior of two groups of masses is presented. The vertical dotted lines indicate the moments when the second discharge is switched on and off, respectively. At the beginning, both discharges are switched off. After the ignition of the acetylene discharge with the addition of hydrogen molecules, the masses 50 and 74 are created and the formation of neutrals follow the periodicity of the dust particle formation. The addition of hydrogen atoms into the acetylene discharge changes the chemical composition of the plasma in such a way that both masses presented in Fig. 4.11 exhibit a decrease in intensity. This is the more pronounced in the case of mass 74 . Immediately after the second discharge is switched off all masses reach their original values. Obviously, the hydrogen atoms injected from the second discharge influence the chemical composition and also the polymerization process. The examination of the whole mass spectrum however shows that the situation is more complicated and that two groups of masses exist. While the signals of one group of masses increase after the injection of hydrogen atoms the second 


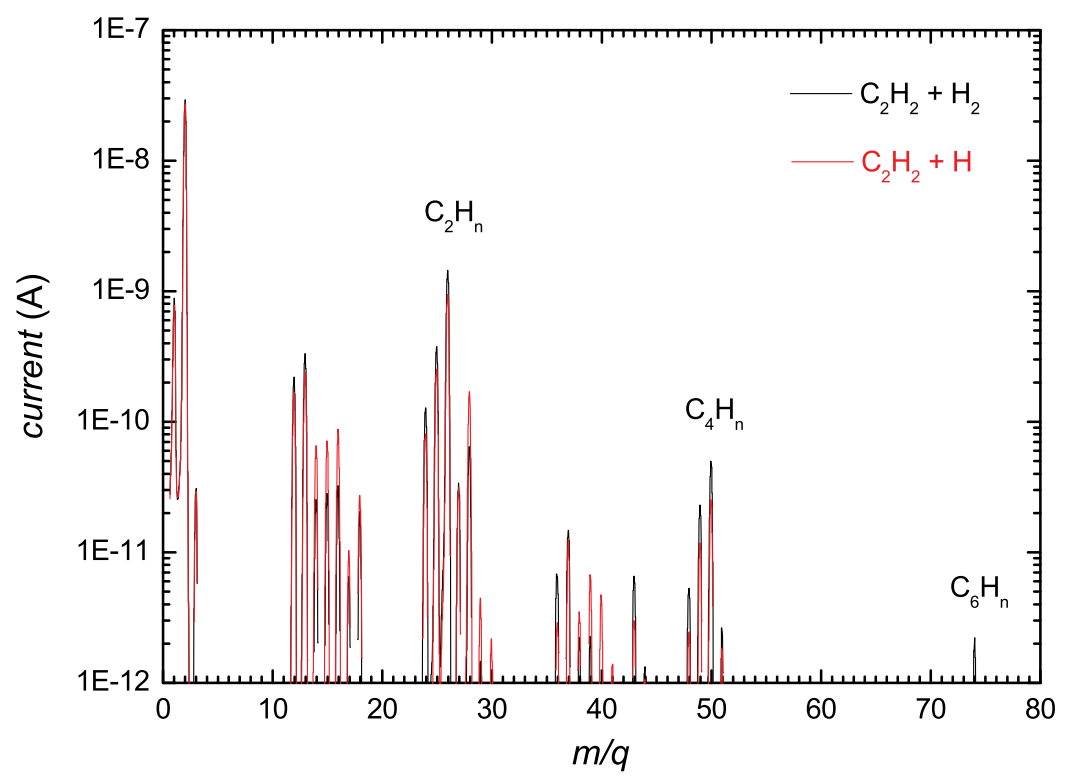

Figure 4.10: The neutrals mass spectrum. Three distinct groups of neutrals in the acetylene mass spectrum can be observed around the masses $m=26$ (acetylene), $m=50$ (biacetylene) and $m=74$ (triacetylene). The black spectrum corresponds to the case when the hydrogen discharge is switched off, the red spectrum to the spectrum measured when the second discharge is switched on under the same discharge operating conditions.

group contains the masses whose intensities decrease after the injection of hydrogen atoms. On the lower graph in Fig. 4.11 the behavior of the mass 78 (benzene, $\left.\mathrm{C}_{6} \mathrm{H}_{6}\right)$ is presented. After injection of hydrogen atoms in the acetylene discharge, the concentration of benzene molecules increases.

The same tendency in the polymerization process of higher hydrocarbon polymers is observed at low concentrations of acetylene when the formation of dust particles is not observed. In such an experiment the flow of the acetylene is reduced to $1 \mathrm{sccm}$ while all other discharge operation conditions are kept constant. The result of this measurement is presented in the Fig. 4.12. After the injection of hydrogen atoms into the acetylene discharge the polymerization process of the higher polymer with the mass 50 is reduced. A similar phenomenon under the same discharge operation conditions is observed for the positive ion with $m / q=50$ and that is presented in Fig. 4.9. It is evident that the polymerization process is interrupted after the addition of hydrogen atoms and it results in a decrease of the concentration of the higher polymers. The influence of hydrogen molecules and atoms on the plasma chemistry on the way experimentally obtained in this work and the suppression of 

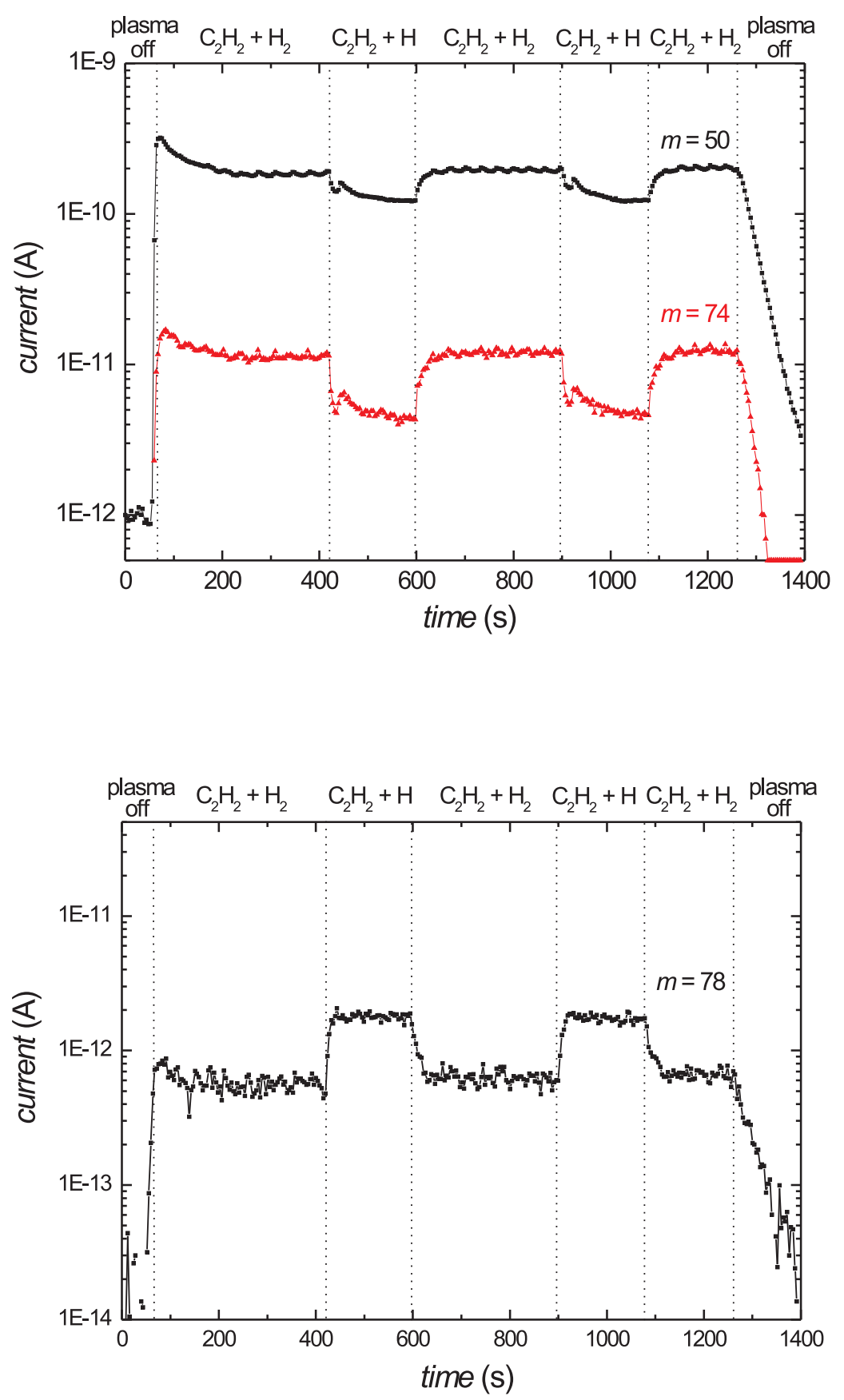

Figure 4.11: The time evolution of the peak intensity corresponding to the masses 50 $\left(\mathrm{C}_{4} \mathrm{H}_{2}\right)$ and $74\left(\mathrm{C}_{6} \mathrm{H}_{2}\right)$ (upper graph) and $78\left(\mathrm{C}_{6} \mathrm{H}_{6}\right)$ (lower graph) after the injection of hydrogen atoms into the acetylene discharge two groups of masses can be distinguished. The vertical dotted lines indicate the moments when the second discharge is switched on and off, respectively. 


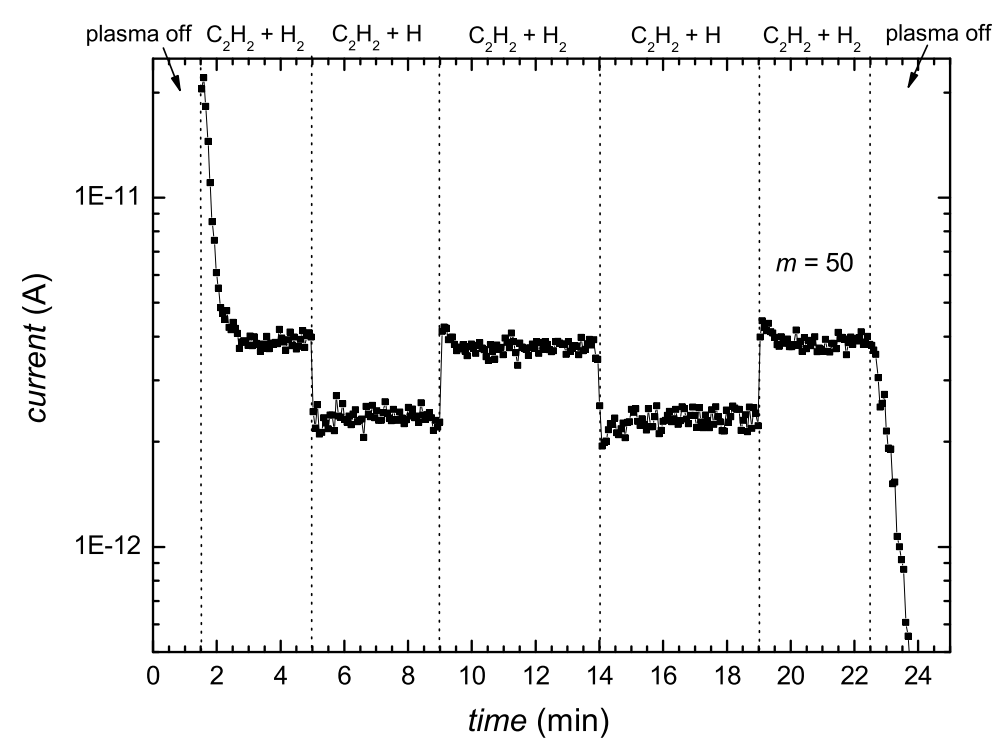

Figure 4.12: The temporal behavior of the mass $m=50$ (biacetylene, $\mathrm{C}_{4} \mathrm{H}_{2}$ ) under conditions where there is no dust formation in the plasma. The vertical dotted lines indicate the moments when the second discharge is switched off and on, i.e. the addition of hydrogen atoms.

higher hydrocarbon ions formation is predicted in the model of hydrocarbon particle nucleation in the acetylene discharge diluted with the molecular hydrogen given by De Bleecker et al [87]. A similar effect of hydrogen atoms on the suppression of particles formation is experimentally observed in silane discharge and reported by Watanabe et al [52]. The suppression of particle growth increases with the degree of silane dilution by hydrogen molecules.

\section{Hydrogen atoms and neutral-neutral chemistry - a possible chemical scenario in suppression of dust particle formation}

Some possible chemical reactions in the formation of larger neutral hydrocarbon precursors and the possible pathways of their destruction by the additionally injected hydrogen atoms in an acetylene discharge are presented in Fig. 4.13. Probably the most important neutral radical in the polymerization process is $\mathrm{C}_{2} \mathrm{H}$. The $\mathrm{C}_{2} \mathrm{H}$ radical can be created from $\mathrm{C}_{2} \mathrm{H}_{2}$ in an electron induced dissociation reaction:

$$
\mathrm{C}_{2} \mathrm{H}_{2}+\mathrm{e}^{-} \rightarrow \mathrm{C}_{2} \mathrm{H}+\mathrm{H}+\mathrm{e}^{-}
$$




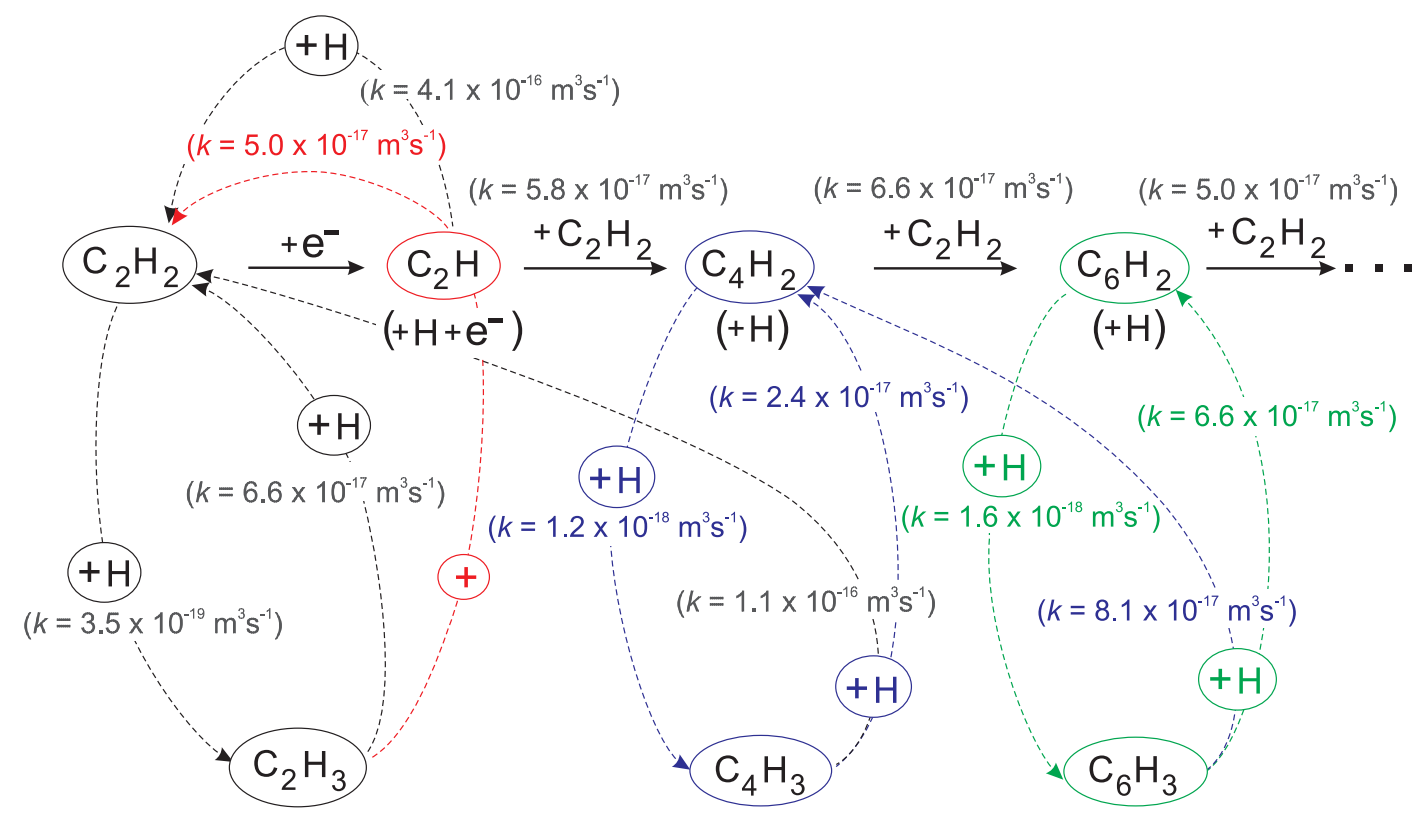

Figure 4.13: The possible chemical scenario in dust particle formation, and the pathways of the growth precursors destruction.

After creation $\mathrm{C}_{2} \mathrm{H}$ which has a threshold energy of $7.5 \mathrm{eV}$ [86] can undergo reactions with the $\mathrm{C}_{2} \mathrm{H}_{2}$ precursor molecules building a biacetylene hydrocarbon neutral:

$$
\mathrm{C}_{2} \mathrm{H}+\mathrm{C}_{2} \mathrm{H}_{2} \rightarrow \mathrm{C}_{4} \mathrm{H}_{2}
$$

The rate coefficient of this reaction is $5.8 \cdot 10^{-17} \mathrm{~m}^{3} \mathrm{~s}^{-1}$ The biacetylene radicals can further react with other $\mathrm{C}_{2} \mathrm{H}_{2}$ precursor molecules building triacetylene $\mathrm{C}_{6} \mathrm{H}_{2}(6.6$ $\left.\cdot 10^{-17} \mathrm{~m}^{3} \mathrm{~s}^{-1}\right)$ :

$$
\mathrm{C}_{4} \mathrm{H}_{2}+\mathrm{C}_{2} \mathrm{H}_{2} \rightarrow \mathrm{C}_{6} \mathrm{H}_{2}
$$

where $\mathrm{C}_{6} \mathrm{H}_{2}$ with the rate coefficient $5.0 \cdot 10^{-17} \mathrm{~m}^{3} \mathrm{~s}^{-1}$ can further polymerize with the precursor molecules giving the higher hydrocarbon polymers. All this masses are found in the neutral mass spectrum of the acetylene discharge presented in Fig. 4.10 .

Hong et al [88] already demonstrated the importance of $\mathrm{C}_{2} \mathrm{H}_{2}$ in the dust particle synthesis. If it can be assumed that actually $\mathrm{C}_{2} \mathrm{H}$ plays a crucial role in the polymerization process or at least at the beginning of the process, then the question is how the additional flux of hydrogen atoms influences the concentration of this radical. Since the neutral mass spectrum demonstrates the decrease of intensity of $\mathrm{C}_{2} \mathrm{H}$ and of most of the masses, it can be supposed that hydrogen atoms destroy more $\mathrm{C}_{2} \mathrm{H}$ radicals then they create. After the injection of additional flux of hydrogen 
atoms, following reactions between the $\mathrm{H}$ atoms and the created acetylene radicals can occur $\left(k=4.1 \cdot 10^{-16} \mathrm{~m}^{3} \mathrm{~s}^{-1}\right)$ :

$$
\mathrm{C}_{2} \mathrm{H}+\mathrm{H} \rightarrow \mathrm{C}_{2} \mathrm{H}_{2} \text {. }
$$

This is likely to be the most important step because of the relatively high cross section and the relatively large concentration of $\left(\mathrm{C}_{2} \mathrm{H}\right)$. The creation of vinyl radical $\mathrm{C}_{2} \mathrm{H}_{3}$ occurs by hydrogen insertion in the $\mathrm{C}_{2} \mathrm{H}_{2}$ molecule $\left(k=3.5 \cdot 10^{-19} \mathrm{~m}^{3} \mathrm{~s}^{-1}\right)$ :

$$
\mathrm{C}_{2} \mathrm{H}_{2}+\mathrm{H} \rightarrow \mathrm{C}_{2} \mathrm{H}_{3},
$$

where $\mathrm{C}_{2} \mathrm{H}_{3}$ can further react with hydrogen $\left(k=6.6 \cdot 10^{-17} \mathrm{~m}^{3} \mathrm{~s}^{-1}\right)$ :

$$
\mathrm{C}_{2} \mathrm{H}_{3}+\mathrm{H} \rightarrow \mathrm{C}_{2} \mathrm{H}_{2}+\mathrm{H}_{2},
$$

or with another $\mathrm{C}_{2} \mathrm{H}$ radical $\left(k=5.0 \cdot 10^{-17} \mathrm{~m}^{3} \mathrm{~s}^{-1}\right)$ :

$$
\mathrm{C}_{2} \mathrm{H}+\mathrm{C}_{2} \mathrm{H}_{3} \rightarrow 2 \cdot \mathrm{C}_{2} \mathrm{H}_{2} .
$$

but in both reactions $\mathrm{C}_{2} \mathrm{H}_{3}$ is removed from the polymerization chain.

The insertion of hydrogen atom in the $\mathrm{C}_{4} \mathrm{H}_{2}$ radical $\left(k=1.2 \cdot 10^{-18} \mathrm{~m}^{3} \mathrm{~s}^{-1}\right)$ :

$$
\mathrm{C}_{4} \mathrm{H}_{2}+\mathrm{H} \rightarrow \mathrm{C}_{4} \mathrm{H}_{3}
$$

a $\mathrm{C}_{4} \mathrm{H}_{3}$ radical is formed, which is destroyed in reaction with an $\mathrm{H}$ atom $(k=$ $\left.1.1 \cdot 10^{-16} \mathrm{~m}^{3} \mathrm{~s}^{-1}\right)$ :

$$
\mathrm{C}_{4} \mathrm{H}_{3}+\mathrm{H} \rightarrow 2 \mathrm{C}_{2} \mathrm{H}_{2},
$$

giving two acetylene molecules.

Inserting an $\mathrm{H}$ atom in the $\mathrm{C}_{6} \mathrm{H}_{2}$ radical $\left(k=1.6 \cdot 10^{-18} \mathrm{~m}^{3} \mathrm{~s}^{-1}\right)$ :

$$
\mathrm{C}_{6} \mathrm{H}_{2}+\mathrm{H} \rightarrow \mathrm{C}_{6} \mathrm{H}_{3},
$$

leads to the formation of a $\mathrm{C}_{6} \mathrm{H}_{3}$ radical which can be further destroyed in reactions with hydrogen atoms:

$$
\begin{gathered}
\mathrm{C}_{6} \mathrm{H}_{3}+\mathrm{H} \rightarrow 3 \mathrm{C}_{2} \mathrm{H}_{2}+\mathrm{H}_{2}, \\
\mathrm{C}_{6} \mathrm{H}_{3}+\mathrm{H} \rightarrow \mathrm{C}_{4} \mathrm{H}_{2}+\mathrm{C}_{2} \mathrm{H}_{2} .
\end{gathered}
$$

On this way, probably through the reactions with hydrogen atoms, the growth precursor particles are destroyed and the further synthesis of the higher hydrocarbon 
polymers is quite reduced resulting in disabling of dust particle formation after injection of an additional flux of hydrogen atoms. So, added hydrogen atoms act in the very early stage of particle formation, in the 'chemical' phase of the particle genesis.

The understanding of possible reactions leading in aromatic hydrocarbon ring structure formation is even more delicate because of the lack of experimental data. It may start with the insertion of one hydrogen atom in a $\mathrm{C}_{2} \mathrm{H}_{2}$ molecule. After that the radical $\mathrm{C}_{2} \mathrm{H}_{3}$ can further react with another $\mathrm{C}_{2} \mathrm{H}_{2}$ molecule. The reaction with one more $\mathrm{C}_{2} \mathrm{H}_{2}$ molecule gives $\mathrm{C}_{6} \mathrm{H}_{6}$ with release of one hydrogen atom as it is presented in equation (4.41) or a radical $\mathrm{C}_{6} \mathrm{H}_{7}$ which will, in reaction of abstraction with one hydrogen atom, transform in a benzene ring as it is presented in equation(4.42). This pathway is represented by the reactions:

$$
\mathrm{C}_{2} \mathrm{H}_{2} \stackrel{+\mathrm{H}}{\longrightarrow} \mathrm{C}_{2} \mathrm{H}_{3} \stackrel{+\mathrm{C}_{2} \mathrm{H}_{2}}{\longrightarrow} \mathrm{C}_{4} \mathrm{H}_{5} \stackrel{+\mathrm{C}_{2} \mathrm{H}_{2}}{\longrightarrow} \mathrm{C}_{6} \mathrm{H}_{6}+\mathrm{H}
$$

or by:

$$
\mathrm{C}_{6} \mathrm{H}_{7} \stackrel{+\mathrm{H}}{\longrightarrow} \mathrm{C}_{6} \mathrm{H}_{6}+\mathrm{H}_{2} .
$$

The pathway presented with the chain of reactions in equation (4.41) is also proposed to be the first step in the production of polyaromatic hydrocarbons in the interstellar medium in the model given by Allamandola et al [89].

In another, probably more likely channel is the reaction chain starting with the $\mathrm{C}_{2} \mathrm{H}$ radical created in reaction (4.29). In two successive reactions with $\mathrm{C}_{2} \mathrm{H}_{2}$ molecules again the benzene ring is formed. It is more likely then reactions (4.41) and (4.42) because of the high concentration of $\mathrm{C}_{2} \mathrm{H}_{2}$ (parent molecules) in the system.

$$
\mathrm{C}_{2} \mathrm{H} \stackrel{+\mathrm{C}_{2} \mathrm{H}_{2}}{\longrightarrow} \mathrm{C}_{4} \mathrm{H}_{3} \stackrel{+\mathrm{C}_{2} \mathrm{H}_{2}}{\longrightarrow} \mathrm{C}_{4} \mathrm{H}_{5} \stackrel{+\mathrm{C}_{2} \mathrm{H}_{2}}{\longrightarrow} \mathrm{C}_{6} \mathrm{H}_{6}+\mathrm{H}
$$

Even though the higher masses, as for example 50 and 74, decrease in intensity after the injection of additional amount of hydrogen atoms, benzene $\mathrm{C}_{6} \mathrm{H}_{6}$ with mass 78 increases after injection probably because it is a stable molecule for instance of other intermediates created during this process. Thus, the stability of the benzene molecule pushes the chemical reactions in the direction of its creation.

\section{The role of anions}

It is already mentioned that negative ions play a crucial role in the dust particle formation since they are confined by the positive plasma potential within the plasma bulk. The reactions of $\mathrm{C}^{-}$and $\mathrm{C}_{\mathrm{n}}^{-}$anions with hydrogen molecules and atoms are 
systematically studied in [90]. It is found that the carbon $\mathrm{C}^{-}$anions do not react with the hydrogen molecules while $\mathrm{C}^{-}$readily reacts with the hydrogen atoms with a rate constant $k \sim 7 \cdot 10^{-10} \mathrm{~cm}^{3} \mathrm{~s}^{-1}$ approximately equal for all carbon anion chain lengths $\mathrm{C}_{\mathrm{n}}^{-}$. For smaller $\mathrm{C}^{-}(n \leq 6)$ anions the associative detachment:

$$
\mathrm{C}_{\mathrm{n}}^{-}+\mathrm{H} \rightarrow \mathrm{C}_{\mathrm{n}} \mathrm{H}+\mathrm{e}^{-}
$$

is found to be the only reaction channel. Experimentally is predicted that at $n \sim 13$ only the reaction of association should be observed:

$$
\mathrm{C}_{\mathrm{n}}^{-}+\mathrm{H} \rightarrow \mathrm{C}_{\mathrm{n}} \mathrm{H}^{-} .
$$

In the reaction of $\mathrm{C}_{\mathrm{n}}^{-}$with hydrogen molecules ionic products are not found, but the associative detachment:

$$
\mathrm{C}_{\mathrm{n}} \mathrm{H}^{-}+\mathrm{H} \rightarrow \mathrm{C}_{\mathrm{n}} \mathrm{H}_{2}+\mathrm{e}^{-},
$$

is observed to be the only channel. The rate coefficient for these reactions are approximately equal to the rate coefficients for the reactions of bare $\mathrm{C}^{-}$anions with hydrogen atoms, except that for $n=2$, which has the twice that rate.

\subsubsection{Conclusions}

This section presents the results of the investigation of the temporal behavior of dust particle formation and the suppression of dust particle formation after the injection of additional flux of hydrogen atoms into the acetylene plasma. For this investigation a double-plasma experiment was employed. An inductively coupled plasma is used for the dust particle creation while the spatial afterglow of an ultra high frequency plasma is used as a source of hydrogen atoms.

The temporal behavior of the dust particle formation is monitored by measuring the intensity of the scattered laser light. These measurements revealed that as soon as the hydrogen atoms are injected into the acetylene plasma further dust particle formation is completely suppressed.

The mass spectra of positive ions and neutrals exhibits the same periodicity as the formation of dust particles up to the moment when the additional flux of hydrogen atoms is injected. After that moment there is no periodicity in the mass creation and destruction. It can be also noticed that the spectra contains two groups of masses. In one group are the masses whose intensity decreases and in the other are the masses whose intensity increases after the injection of hydrogen atoms. 
The results can be understood in terms of the destruction of the precursor radical $\mathrm{C}_{2} \mathrm{H}$, driving the polymerization reaction, via:

$$
\mathrm{C}_{2} \mathrm{H}+\mathrm{H} \rightarrow \mathrm{C}_{2} \mathrm{H}_{2},
$$

thus suppressing the sequential growth of large molecular species. 


\section{Chapter 5}

\section{The role of hydrogen atoms in amorphous hydrogenated carbon (a-C:H) film formation}

\subsection{Introduction}

Amorphous hydrogenated carbon films (a-C:H) with their wide variety of properties have attracted a lot of interest. Depending on the deposition conditions they can vary from hard diamond-like to soft polymer-like carbon films. They found a lot of applications due to their extraordinary properties as high mechanical hardness, high wear resistance, low friction coefficient, optical transparency in visible and infrared range, chemical inertness etc. They are, for example, widely used as protective coatings on optical devices, as wear resistant coatings or for coating the implantants, for computer hard disc drivers etc. [91, 92, 93].

Hydrogen molecules and atoms are created inherently in every hydrocarbon discharge due to the dissociation of precursor molecules and stay in the discharge long enough to play an important role in the plasma chemistry and in the surface processes contributing significantly to the flux of radicals impinging onto the surface. The growth of the film is the net result of the interplay between the etching process and the more complicated deposition process. The hydrogen atoms acting together with ions during the growth of the film can activate or deactivate the surface through the creation of dangling bonds [94, 95] or their saturation, leading to the enhancement (synergistic effect) or reduction (anti-synergistic effect) $[95,96]$ of the growth speed, respectively.

The growth synergism is explained by the dangling bonds formation. They are created via surface bounded hydrogen abstraction by incoming hydrogen atoms. The dangling bonds then serve as adsorption sites for incoming $\mathrm{CH}_{3}$ radicals [94, 95]. On the other hand, Jacob [96] and Schwarz-Selinger et al [95] observed an anti- 
synergistic effect. Additional ion bombardment changes both growing and etched surface. Along the whole ion penetration range bonded hydrogen atoms are displaced. Displaced hydrogen atoms then recombine forming $\mathrm{H}_{2}$ molecules which then diffuse and desorb leaving behind a harder film due to lowering of the hydrogen content.

Physical sputtering by hydrogen ions $[97,98]$ and chemical erosion of a:C-H films [99] by $\mathrm{H}$ atoms were investigated in numerous experiments employing thermal hydrogen atom beams. Significant attention is also paid in understanding the role ions play in combination with atomic hydrogen during film etching [100] and plasma deposition of the hydrocarbon films [101]. Two effects are described in the literature. Vietzke et al. [102] observed ion-induced enhancement of graphite etching at elevated temperatures by atomic hydrogen even though graphite basal planes are very inert to atomic hydrogen addition. Ions during bombardment create dangling bonds, chemisorption sites for impinging atomic hydrogen. As a consequence a hydrogen-rich surface layer is created which is the precursor for volatile products leading to an enhancement of the atomic hydrogen erosion yield.

On the other hand, von Keudell and Jacob [103] observed ion-induced reduction in etching speed of polymer-like hydrogenated carbon films at elevated temperatures. Predominantly bonded hydrogen is displaced due to ion bombardment leading to cross linking of the carbon network, i.e. ions transform $s p^{3}$ hybridized carbon groups into $s p^{2}$ hybridized carbon groups and destroy the precursor for erosion products.

Usually the films are deposited in low temperature plasmas. The plasma is a complex system of particles with different energies and reactivities impinging simultaneously onto the surface. Under such conditions it is very difficulty to recognize the microscopic growth mechanisms responsible for the film growth and to determine the rate coefficients or the cross sections of different processes. One approach in resolving this complex problem is to use quantified beams of different kind of particles in studding the individual growth process simulating the artificial plasma.

The structure of this part is following. Section 5.1 contains the introduction. The results of the influence of hydrogen atoms in combination with $\mathrm{Ar}^{+}$ions on the deposition/etching speed under plasma conditions are presented in section 5.2. The influence of hydrogen atoms on the film properties - optical constant and film structure are presented in section 5.3. The section 5.4 brings the results about up to now still not enough investigated film growth in presence of dust particles. Some conclusions, based on these results, are given at the end of this chapter. 


\subsection{Film growth}

\subsubsection{Influence of hydrogen atoms on the deposition speed}

The influence of the hydrogen atoms on the growth speeds of the thin films under plasma conditions is studied in details in experiments using a surface wave sustained discharge. Here a flow rate of $5 \mathrm{sccm}$ of hydrogen molecules/atoms is added into the $50 \mathrm{sccm}$ expanded argon plasma and the $75 \mathrm{sccm}$ methane. The applied power is $300 \mathrm{~W}$ and the pressure in the chamber is $10^{-3}$ mbar.

The experiment consists of three steps. In the first step the film is deposited in the argon/methane plasma. In the second step molecular hydrogen is added to the argon/methane plasma. In the third step the hydrogen plasma is ignited and molecular hydrogen and hydrogen atoms are added into the argon/methane plasma. After each deposition process the wafer is replaced by a new empty one.

The measured growth rates are presented in Fig. 5.1. The black squares represent the growth rate for the case when no additional hydrogen (molecular or atomic) is injected into the reaction chamber. The position of the expanding plasma and the wafer and the common profile of the films deposited in this arrangement is presented on photo in Fig. 2.12. The growth rate along the center line is slightly reduced when an additional flux of molecular hydrogen is introduced in the reaction chamber (red circles in Fig. 5.1) through the glass cylinder 2 (see Fig. 2.1). In the plasma ball region weak dissociation and ionization processes occur leading to the creation of hydrogen atoms and molecular and atomic hydrogen ions. They react with the surface during the film growth causing a small reduction in the growth rate. As soon as hydrogen atoms are added to the argon/methane plasma, i.e. as soon as the second discharge is switched on, the corresponding growth rate is significantly reduced (blue triangles in Fig. 5.1).

The net growth rate that is observed in the experiment is the gross growth rate minus the etching rate:

net growth rate $=$ gross growth rate - etching rate.

The reduction of the observed growth rate that occurs in the experiment as soon as the second discharge is switched on can be explained therefore in two ways: the additional flux of hydrogen atoms leads to an increase of the etch rate or/and to a decrease of the gross growth rate. Both effects can contribute to the reduction of the net growth rate observed. The additional flux of hydrogen atoms can enhance the effect of chemical erosion which will increase the etching rate [104] and/or it can passivate dangling bonds on the surface which will decrease the gross growth rate. 


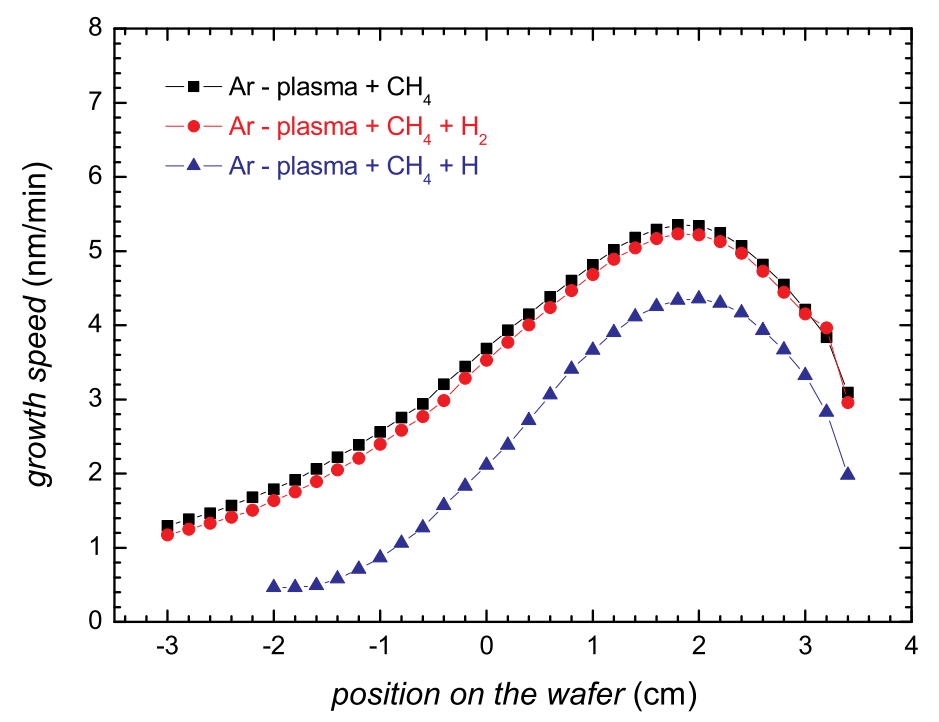

Figure 5.1: Synergistic effect in the growth speed in argon/methane plasma: the growth rate for the case argon/methane plasma (full black squares), an additional flux of molecular hydrogen is introduced in the reaction chamber (full red circles), the atomic hydrogen is added to the argon/methane plasma (full blue triangles).

One has to take into account that hydrogen atoms can passivate the surface. The cross section for the addition of hydrogen atoms to a dangling bond, $\sigma_{\text {addition } \mathrm{H}}=1.3$ $\AA^{2}$, is only slightly lower than the cross section $\sigma_{\text {addition, } \mathrm{CH}_{3}}$ for the addition of $\mathrm{CH}_{3}$ radicals to a dangling bond ( $\sigma_{\text {addition, } \mathrm{CH}_{3}}$ ranges between 2.4 and $5.4 \AA^{2}$ [105] and thus much larger than $\sigma_{\text {abstraction } \mathrm{H}}=0.05 \AA^{2}$ [99]). The situation is different however as soon as other mechanisms - as for example $\mathrm{Ar}^{+}$ions - lead to the formation of dangling bonds. In this case the addition of hydrogen atoms can lead to a reduction of the gross growth rate.

The additional flux of hydrogen atoms can lead to change in the gross growth rate. It can in principle enhance or reduce the gross growth rate. von Keudell et al [104], for instance, showed in experiments with beams of hydrogen atoms and $\mathrm{CH}_{3}$ radicals that the sticking probability of $\mathrm{CH}_{3}$ radicals is enhanced due to the simultaneous action of hydrogen atoms. In principle, this effect could explain the discrepancy in the enhancement of the etching rate and the reduction of the growth rate discussed above. In the present experiment however such a mechanism is unlikely to happen. While the cross section for the abstraction of the surface bonded hydrogen - and thus for the creation of the dangling bonds - by incoming hydrogen atoms is $\sigma_{\text {abstraction, } \mathrm{H}}=$ $0.05 \AA^{2}$ the cross section for the abstraction of surface bonded hydrogen by incoming 


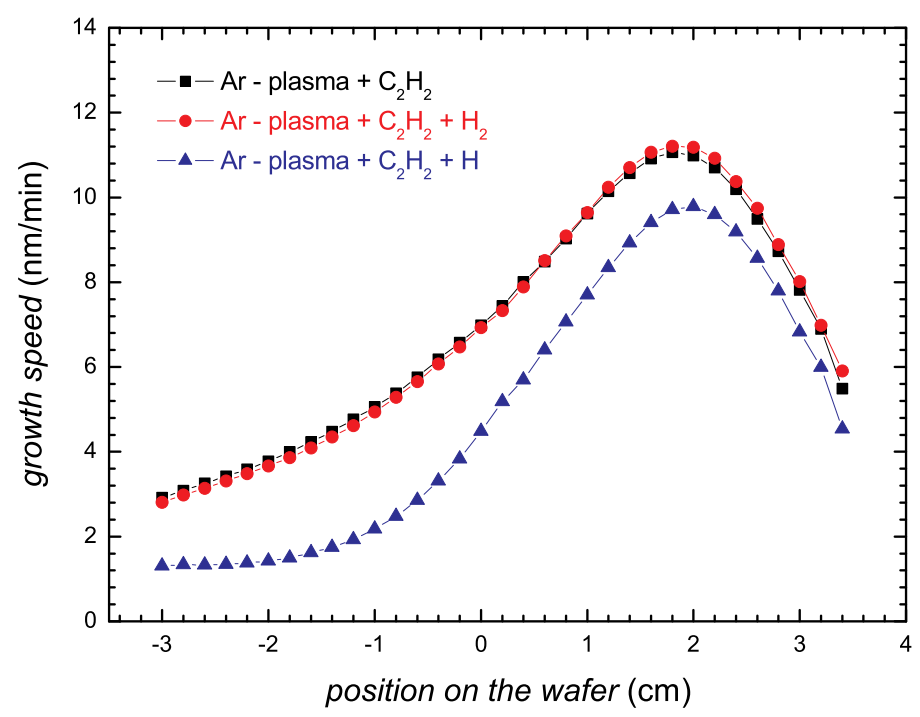

Figure 5.2: The growth rate for the case of an argon/acetylene plasma (black squares) is slightly reduced when an additional flux of the hydrogen molecules is introduced in the reaction chamber (red circles). After addition of the hydrogen atoms to the argon/acetylene plasma the corresponding growth rate is significantly reduced (blue triangles).

$\mathrm{CH}_{3}$ radicals is more than one order of magnitude smaller, namely $\sigma_{\text {abstraction, } \mathrm{CH}_{3}}=$ $1.5 \cdot 10^{-3} \AA^{2}$. In experiments which use $\mathrm{CH}_{3}$-beam in combination with a H-beam the additional flux of hydrogen atoms will therefore lead to an increase of the growth rate since the hydrogen atoms activate the surface via the formation of dangling bonds.

\subsubsection{Role of the precursor gas}

Fig. 5.2 shows the results of an experiment that is performed in the same way as described above and under the same experimental conditions, only instead of methane the acetylene is used as a precursor gas. The film growth rate in the argon/acetylene discharge is higher then in the argon/methane discharge but the reduction of the ratio of growth speeds that is observed in the argon/acetylene discharge in presence of hydrogen atoms is the same as in the argon/methane discharge.

In the acetylene plasma the most important radical is probably $\mathrm{C}_{2} \mathrm{H}$. Since the sticking coefficient of this radical is close to unity the gross growth rate should not be affected too much neither by the activation nor by the passivation of the surface. After $\mathrm{C}_{2} \mathrm{H}$ chemisorbs on the surface the $\mathrm{C} \equiv \mathrm{C}$ triple bond can be easily broken by 
incoming hydrogen atoms and then new $\mathrm{C}-\mathrm{H}$ bonds are created.

The comparison of the absolute growth speed of the film deposited in methane and the film deposited in acetylene demonstrates the influence of the hybridization of the precursor gas molecule, i.e. the hybridization of the radical growth precursors on the growth speed [106]. The hybridization of the radical growth precursor means the density of the dangling bonds. Therefore, as the precursor radical is more unsaturated the growth speed is higher. In this two plasmas on one side is the maximally unsaturated $\mathrm{C}_{2} \mathrm{H}$ radical while on the other side is the maximally saturated $\mathrm{CH}_{3}$ radical. The hybridization of the precursor gas molecules consequently affects the film structure, this being presented in the part about the film structure.

\subsubsection{Influence of hydrogen atoms on the etching speed}

The possible contribution of the hydrogen atoms to the reduction of the growth speed is investigated in a purely etching experiment. The thin hydrocarbon films are first deposited in the GEC reactor and then carried into the second chamber where they are exposed to the different kind of plasmas. The experiment consists of four steps, the results of this experiment being presented in Fig. 5.3. For each step a new wafer with a deposited film is used.

In the first step the film is exposed only to the afterglow of the hydrogen discharge (green diamonds in Fig. 5.3). The etching rate is very small in this case and has a maximum value of about $0.1 \mathrm{~nm} / \mathrm{min}$. Such a chemical etching of films by hydrogen atoms has already been investigated in experiments with thermal hydrogen atom beam [99]. From those experiments it is known that the chemical etching by hydrogen atoms is temperature activated and that this process has a small etching rate at room temperature.

In the next step the deposited film is exposed to the expanding argon plasma only (black squares in Fig. 5.3). The $\mathrm{Ar}^{+}$ions will displace - within their penetration range - predominantly bonded hydrogen atoms which then recombine and form hydrogen molecules. The latter diffuse to the surface and desorb there [104]. The argon ions displace rather hydrogen atoms than carbon atoms since the threshold energy for displacement of hydrogen atom $(2.5 \mathrm{eV})$ is much smaller than the corresponding value for the displacement of carbon atom $(25 \mathrm{eV})$. The etching rate in this experiment is also small, with a maximum value of about $0.45 \mathrm{~nm} / \mathrm{min}$.

The next etching experiment is performed again with the expanding argon plasma. But this time molecular hydrogen is added. This etching profile is represented with red circles in Fig. 5.3. The etching rate is slightly increased but it is still of the same order of magnitude as in the two previous experiments, reaching a maximum 


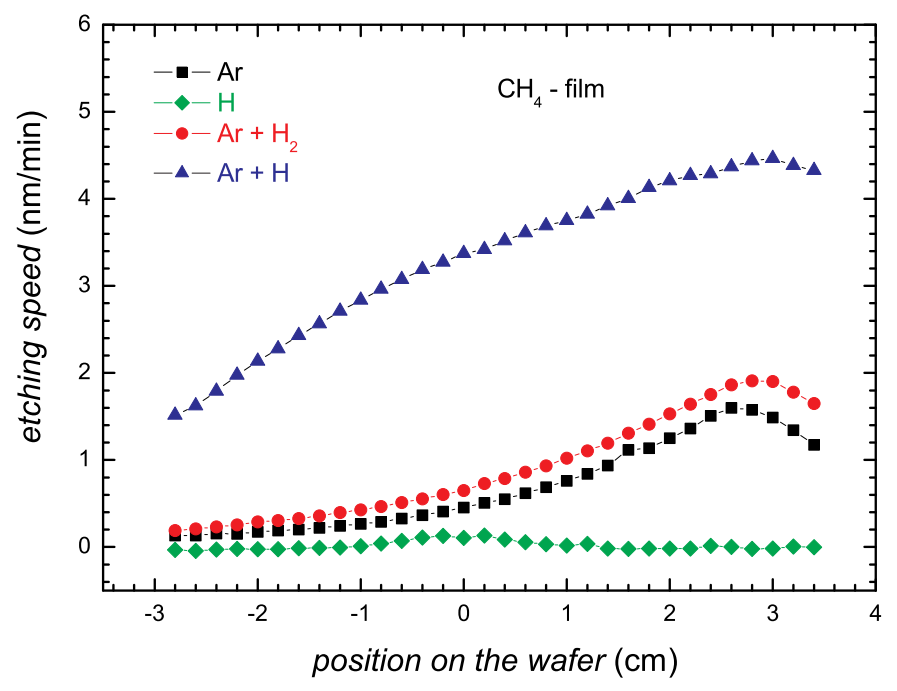

Figure 5.3: Ellipsometric data for the etching of a-C:H film with an optical constant $\tilde{n}=1.60-i \cdot 0.001$ showing a synergistic effect in the etching speed. The black squares denote etching of the film by a flux of $\mathrm{Ar}^{+}$ions, the green diamond etching by $\mathrm{H}$ atoms, the red circles by $\mathrm{Ar}^{+}$and $\mathrm{H}_{2}$ molecules and the blue triangles by simultaneous impact of $\mathrm{Ar}^{+}$ions and $\mathrm{H}$ atoms.

value of about $0.75 \mathrm{~nm} / \mathrm{min}$. The small increase in the etching rate comes form the simultaneous reaction of small amount of $\mathrm{Ar}^{+}$ions and hydrogen atoms, which are created via the dissociation of $\mathrm{H}_{2}$ molecules in the plasma ball region.

In the last step the second discharge operating with molecular hydrogen is ignited. The etching rate measured in this experiment is by far higher then the sum of all individual etching rates obtained in the previous steps. This corresponds to etching profile represented with blue triangles in Fig. 5.3. It is increased nearly by one order of magnitude and reaches a maximum value of $3.4 \mathrm{~nm} / \mathrm{min}$. The enhancement of the etching rate due to the simultaneous action of $\mathrm{Ar}^{+}$ions and additional hydrogen atoms is explained by the process of chemical sputtering. Incoming hydrogen atoms react with dangling bonds created by the simultaneous $\mathrm{Ar}^{+}$bombardment not only on the surface but also within the penetration range of the ions creating in this way volatile $\mathrm{C}_{\mathrm{x}} \mathrm{H}_{\mathrm{y}}$ species. This species then diffuse to the surface and desorb there. This process does not occur only at the surface but also within the whole ion penetration range.

The etching experiment is also performed with films having a higher optical constant, $\widetilde{n}=1.75-\mathrm{i} \cdot 0.009$, i.e. a lower hydrogen content. All etching rates are reduced 
as it is expected according to [104]. Hydrogen-richer surfaces are more susceptible to etching by hydrogen atoms because they have better precursor structures for volatile products.

This experiment shows that the enhancement of the etching rate - due to the action of the second discharge - is large enough to explain the reduction of the growth rate observed in Fig. 5.1. The increase of the etching rate is even larger than the decrease of the growth rate. The growth rate in Fig. 5.1 is reduced from about $3.3 \mathrm{~nm} / \mathrm{min}$ (when molecular hydrogen is added) to $1.8 \mathrm{~nm} / \mathrm{min}$, respectively, when the hydrogen discharge was switched on. The difference in the growth rates is smaller than the difference in the etch rates obtained in the last experiment (3.3 $\mathrm{nm} / \mathrm{min}$ and $0.6 \mathrm{~nm} / \mathrm{min}$ ) when the hydrogen discharge is used together with the expanding argon plasma. A reasonable explanation for this discrepancy is that the expanding argon plasma is changed as soon as methane is added to the vessel. Therefore one cannot directly compare the results from Fig. 5.1 with those in Fig. 5.2. Nevertheless the latter experiment clearly shows that the enhancement of the chemical erosion dominates the reduction of the net growth rate observed in Fig. 5.1 and Fig. 5.2.

\subsubsection{Dependence on ion energy}

To change the ion energy during film deposition, an antenna is introduced into the glass cylinder 1 (see Fig. 2.1) and a pulsed (7 kHz) DC bias voltage (+20/-80 V) is applied while the substrate is grounded. The flow of argon, methane and molecular hydrogen are kept constant, only the applied power to the argon SWSD is increased to $500 \mathrm{~W}$, this being the minimum power to expand the plasma in presence of the applied bias voltage.

In Fig. 5.4 the growth speed, in presence of the bias voltage, of the film deposited in methane (black squares) and in methane with addition of molecular hydrogen (red circles) is presented as a function of the position on the wafer, where position $=0$ means the center of the wafer. The growth speeds of $7 \mathrm{~nm} / \mathrm{min}$ in both cases are two times higher then in the case when no bias voltage is applied. The addition of hydrogen atoms lowers the growth speed of the film (blue triangles) to $1.8 \mathrm{~nm} / \mathrm{min}$. Thus, the synergistic effect also occurs when the bias voltage is additionally applied.

The enhancement in the net growth speed of the films deposited in methane and in the mixture of methane and molecular hydrogen when the bias voltage is additionally applied can be due to the more pronounced ionic flux component so, the number of created dangling bonds at the surface of the growth film via the displacement of the surface bonded hydrogen atoms by the energetic $\mathrm{Ar}^{+}$ions is increased, i.e. the 


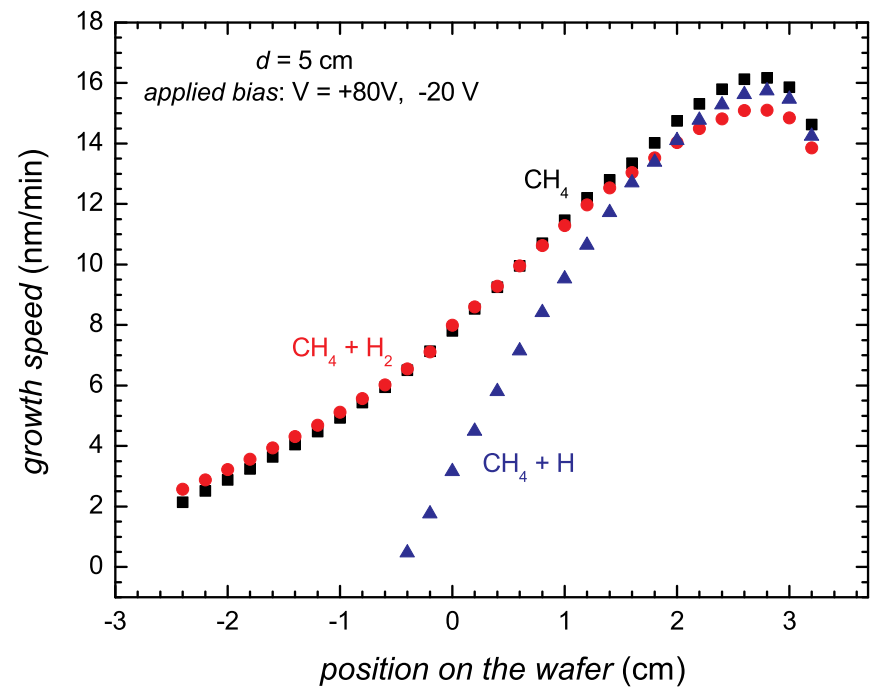

Figure 5.4: The influence of the additionally applied bias voltage on the growth speed: black squares - argon/methane plasma, red circles - the growth speed after the addition of hydrogen molecules, blue triangles - the growth speed after the addition of hydrogen atoms.

number of chemisorption sites for the incoming precursor species is increased. This has as a consequence an increased growth speed.

But, on the other side, the growth speed of $1.8 \mathrm{~nm} / \mathrm{min}$ after the addition of hydrogen atoms (blue triangles in Fig. 5.4) is also achieved when the bias voltage is not applied (blue triangles in Fig. 5.1). In the case when the bias voltage is additionally applied (Fig. 5.4), the energetic ions are very efficient in the production of dangling bonds by displacing the hydrogen atoms on the surface and by breaking the $\mathrm{C}-\mathrm{C}$ bonds along the whole penetration range underneath the surface. The displaced hydrogen atoms are, practically, instantly replaced with the new hydrogen atoms coming from the second discharge and the created dangling bonds on the surface are passivated mostly by incoming hydrogen atoms, what significantly reduces the density of the dangling bonds for the incoming precursor species. The etching speed is increased but it is limited by hydrogen atoms flux. 

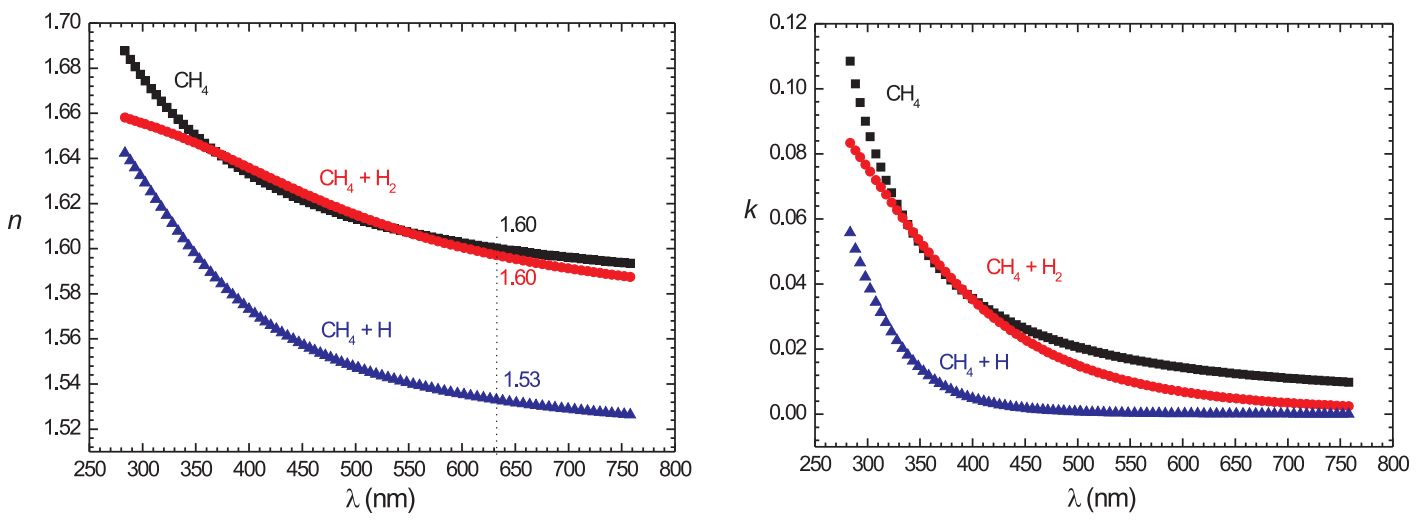

Figure 5.5: Influence of the second discharge on the refractive index, $n$, and on the extinction coefficient, $k$. Black squares: the film deposited in an $\mathrm{Ar} / \mathrm{CH}_{4}$ plasma; red circles: the film deposited in a mixture of $\mathrm{Ar} / \mathrm{CH}_{4}$ and $\mathrm{H}_{2}$; blue triangles: the film deposited in the $\mathrm{Ar} / \mathrm{CH}_{4}$ plasma with additional flux of hydrogen atoms. The indicated values of the refractive index are given for a wavelength $\lambda=632.2 \mathrm{~nm}$.

\subsection{Film properties}

\subsubsection{Influence of hydrogen atoms on the optical constants}

The structure of the film is influenced by the additional flux of hydrogen atoms. Fig. 5.5 shows the changes of the complex refractive index (the real part of the complex refractive index is presented on the left graph and the imaginary part is on the right graph) of the deposited films in methane discharge that are induced by the hydrogen atoms from the second discharge.

The reduction of the refractive index is from $n=1.60$ (pure methane discharge, black squares) and with the addition of the hydrogen molecules (red circles), to $n=$ 1.53 when the second discharge is ignited and the hydrogen atoms are added to the methane discharge (blue triangles). The reduction of the refractive index is small because the growth precursor radicals created in methane, the maximally saturated hydrocarbon $\mathrm{CH}_{\mathrm{n}}$, are also saturated hydrocarbons. Any further hydrogenation can develop only through the hydrogenation of broken $\mathrm{C}-\mathrm{C}$ bonds which have to be first created by the incident ions.

Different are the films deposited in acetylene, the maximally unsaturated hydrocarbon molecule. The refractive index for the films deposited in acetylene and acetylene plus molecular hydrogen is significantly reduced from $n=1.79$ (black squares) to 1.73 (red circles), down to $n=1.59$ (blue triangles) when the second discharge 

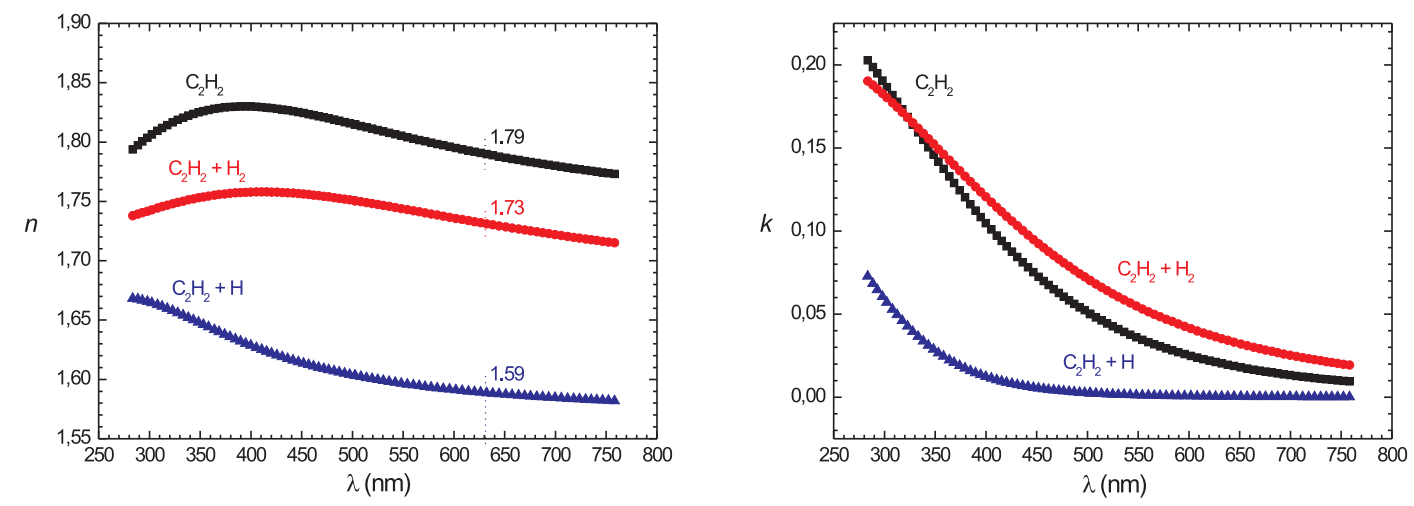

Figure 5.6: Influence of the second discharge on the refractive index, $n$, and on the extinction coefficient, $k$. Black squares: the film deposited in an $\mathrm{Ar} / \mathrm{C}_{2} \mathrm{H}_{2}$ plasma; red circles: the film deposited in a mixture of $\mathrm{Ar} / \mathrm{C}_{2} \mathrm{H}_{2}$ and $\mathrm{H}_{2}$; blue triangles: the film deposited in the $\mathrm{Ar} / \mathrm{C}_{2} \mathrm{H}_{2}$ plasma with additional flux of hydrogen atoms. The indicated values of the refractive index are for $\lambda=632.2 \mathrm{~nm}$ wavelength.

is switched on. In rehybridization $\mathrm{C}_{2} \mathrm{H}$ radicals can produce dangling bonds which are then passivated with hydrogen atoms coming from the second discharge. This is the explanation for the two very different situations in Fig. 5.5 and Fig. 5.6.

The optical constants of the a-C:H films are strongly connected with the film structure and the hydrogen content. The extinction coefficient $k$ at $632.2 \mathrm{~nm}$ is related to the concentration of $s p^{2}$ hybridized carbon atoms in the film [101]. From the right graph in Fig. 5.6 it can be seen that in presence of hydrogen atoms the value of the extinction coefficient is reduced by two orders of magnitude. The reduction of the real part of the refractive index $n$, in the left graph of Fig. 5.6, also indicates a change in the film structure. In an empirical relation higher vales of $n$ correspond to harder, carbon richer, more compact (high density) films. A reduction of $n$ indicates that the films become softer i.e. more polymer-like, richer in hydrogen and less dense [27, 107].

The two graphs in Fig. 5.7 show the changes of the refractive index $n$ (left graph) and the extinction coefficient $k$ (right graph) of the thin films deposited in the methane discharge and in the discharge composed of a mixture of methane and molecular and atomic hydrogen under the influence of an additionally applied bias voltage. While the refractive index has the same value $n=1.72$ in the case of the film deposited in methane (black squares) and in the mixture of methane and molecular hydrogen (red circles), it drops significantly to $n=1.48$ when the hydrogen atoms are injected into the methane discharge. In the first two cases 

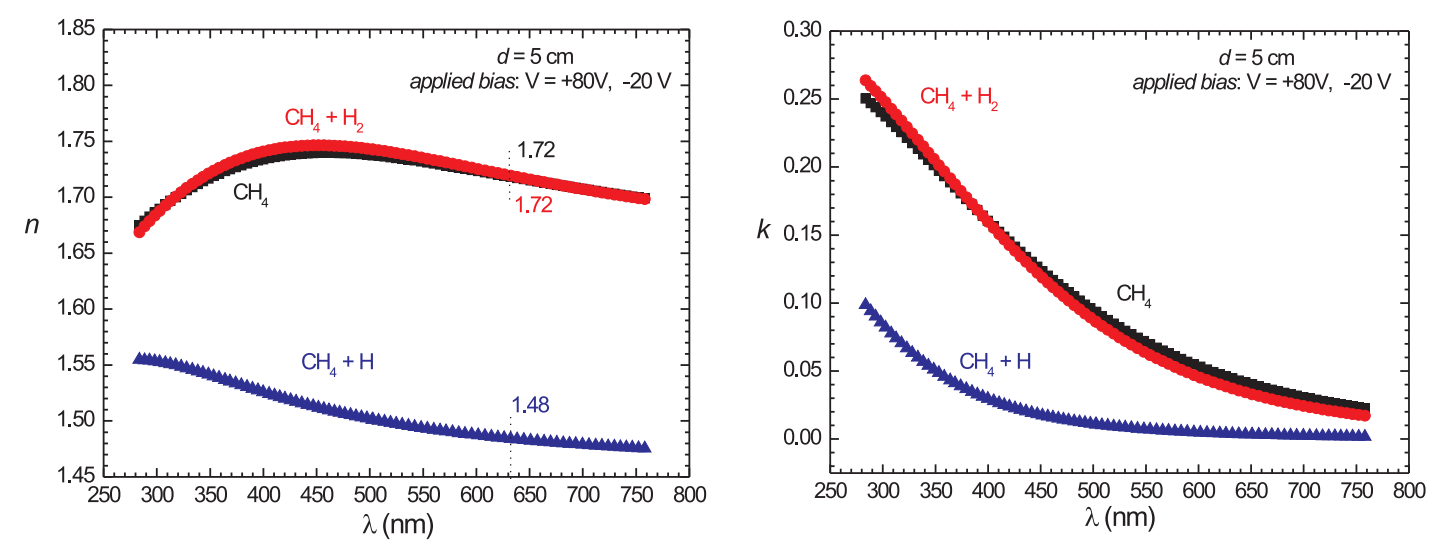

Figure 5.7: The change of the refractive index $n$ and on the extinction coefficient $k$ under the influence of the applied bias voltage. Black squares: the film deposited in an $\mathrm{Ar} / \mathrm{CH}_{4}$ plasma, red circles: the film deposited in a mixture of $\mathrm{Ar} / \mathrm{CH}_{4}$ and $\mathrm{H}_{2}$, blue triangles: the film deposited in the $\mathrm{Ar} / \mathrm{CH}_{4}$ plasma with additional flux of hydrogen atoms. The indicated values of the refractive index are given for $\lambda=632.2 \mathrm{~nm}$ wavelength.

the energetic $\mathrm{Ar}^{+}$ions mainly displace bonded hydrogen atoms on the surface and along their penetration range breaking $\mathrm{C}-\mathrm{H}$ bonds. The hydrogen atoms recombine creating molecules which either stay trapped in the film or desorbe at the surface while the broken bonds between carbon atoms recombine creating $\mathrm{C}-\mathrm{C}$ single bonds or $\mathrm{C}=\mathrm{C}$ double bonds. All this leads in the densification of the deposited film giving a higher value of the refractive index [82].

When an additional flux of hydrogen atoms is injected into the methane plasma, the bonds broken by $\mathrm{Ar}^{+}$ions on the surface and along the ion penetration range are mainly passivated with added hydrogen atoms and the concentration of $\mathrm{C}-\mathrm{H}$ bonds is increased. The decrease in the concentration of $\mathrm{C}-\mathrm{C}$ single bonds is reflected in the lowering of the refractive index value.

\subsubsection{Influence of hydrogen atoms on the film structure}

\section{Bonding in films}

All changes of the film properties presented in the previous section are related to the changes in the film structure. How additional flux of hydrogen atoms change the structure in the case of films deposited in methane can be observed in the Raman spectrum presented in Fig. 5.8.

The Raman spectrum shown in Fig. 5.8 are normalized to unity and are corrected 


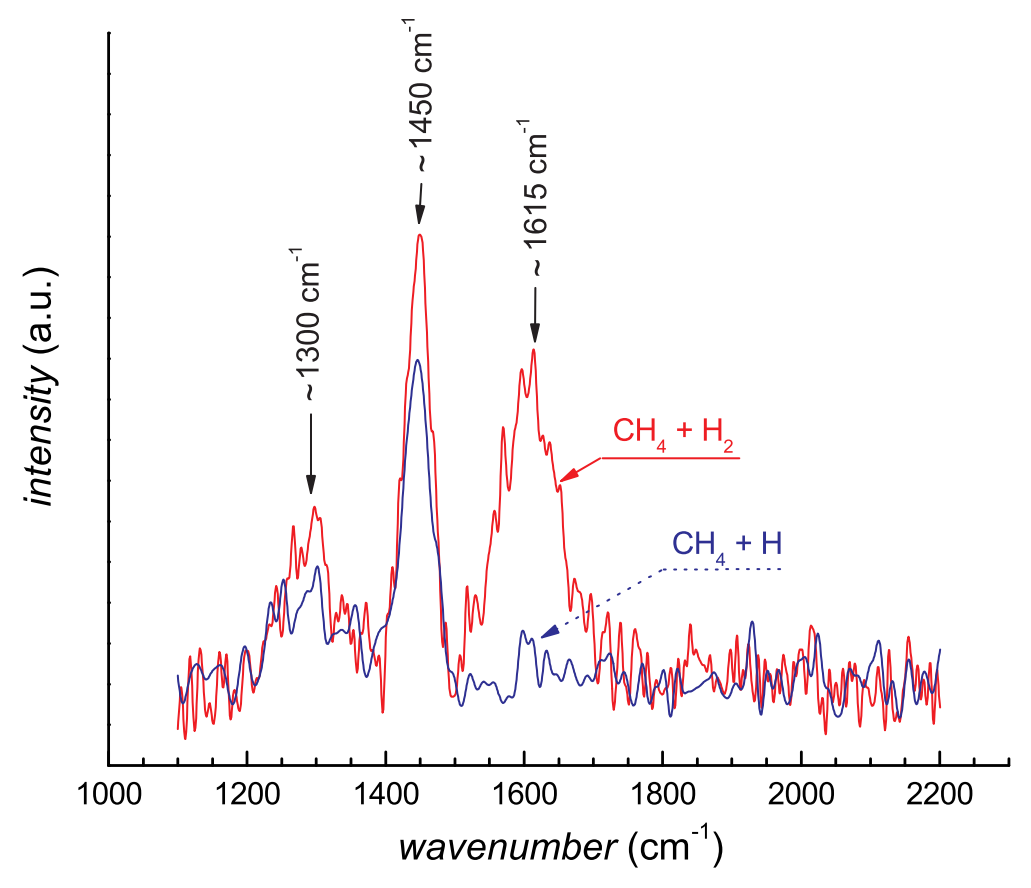

Figure 5.8: Raman spectra of the a-C:H films deposited in argon/methane plasma with addition of hydrogen molecules (red line) and argon/methane plasma with addition of hydrogen atoms (blue line). The Raman spectra are baseline corrected and the intensity of each spectrum is normalized to unity.

by subtracting the background due to luminescence. The most prominent features in Raman spectrum of a-C:H films are the so-called D peak at around $1360 \mathrm{~cm}^{-1}$ and the $\mathrm{G}$ peak at around $1560 \mathrm{~cm}^{-1}$ for visible excitation [27]. The $\mathrm{G}$ peak occurs due to the in-plane bond-stretching motion of all pairs of carbon $s p^{2}$ hybridized atoms at both olefinic and aromatic sites. The D peak is a breathing mode forbidden in perfect graphite but active in presence of disorder in the structure. The D peak arises from aromatic rings and its intensity is directly connected to the presence of sixfold aromatic rings [24]. Another peak often found in the Raman spectrum of such films is located at $1450 \mathrm{~cm}^{-1}$. The origin of this peak is from a trans-polyacetylene phase in the film [28].

Fig. 5.9 shows the influence of hydrogen atoms on the Raman spectrum of films deposited from acetylene. The most pronounced features are the $\mathrm{D}$ peak at around $1300 \mathrm{~cm}^{-1}$ and the $\mathrm{G}$ peak at around $1615 \mathrm{~cm}^{-1}$. When the second discharge is switched on both peaks are disappearing and instead a very weak at $1450 \mathrm{~cm}^{-1}$ becomes visible. The disappearance of both $\mathrm{D}$ and $\mathrm{G}$ peaks shows that the exposure of the film to the additional flux of hydrogen atoms leads to a hydrogenation of 


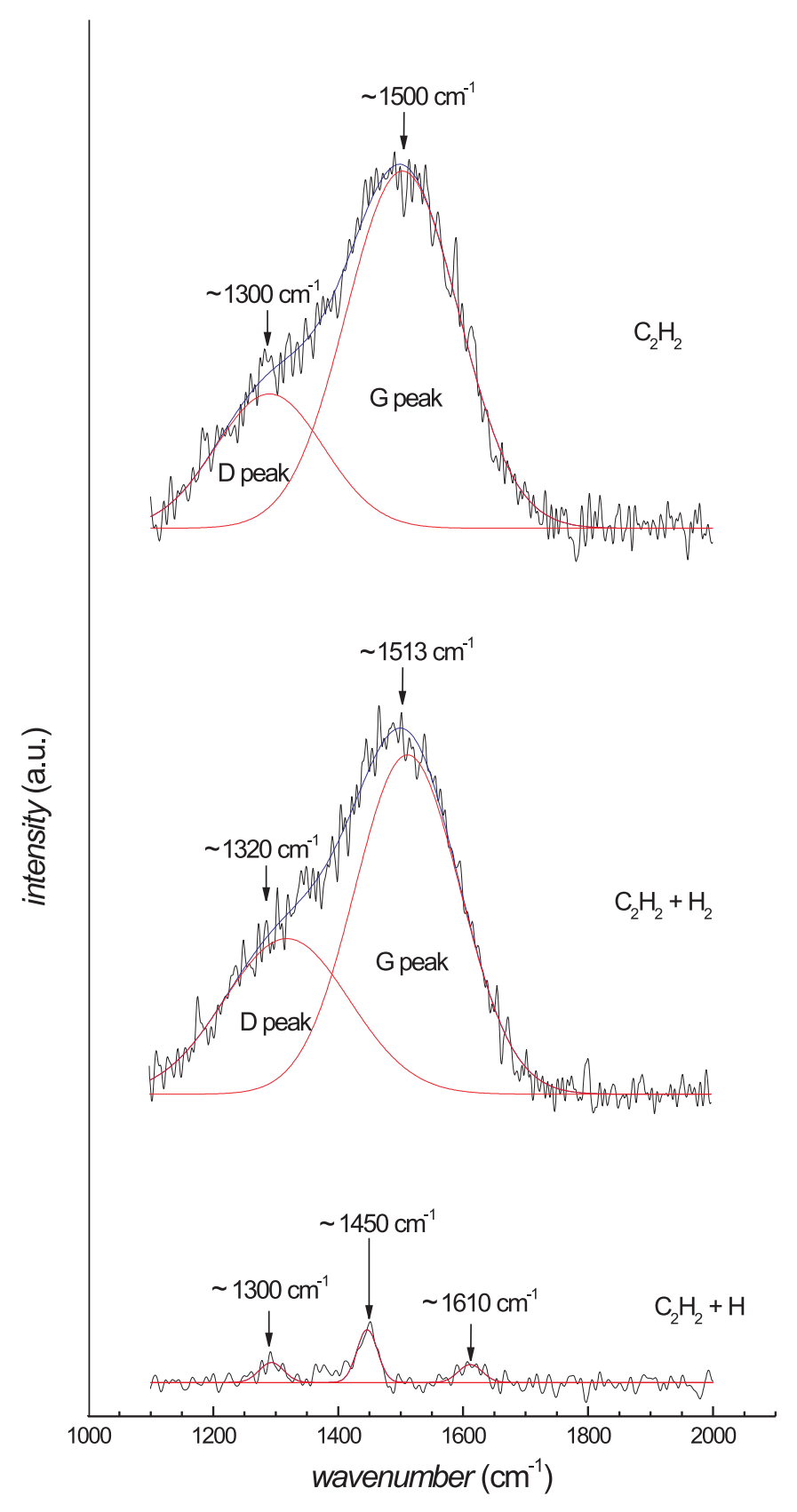

Figure 5.9: Raman spectrum of the a-C:H films deposited in argon/acetylene (black line) and argon/acetylene plasma with addition of hydrogen molecules (red line) and atoms (blue line). The Raman spectra are baseline corrected and the intensity of each spectrum is normalized to unity. 
unsaturated $s p^{2}$ hybridized carbon sites resulting in a gain of $s p^{3}$ hybridized carbon groups and an incorporation of hydrogen atoms into the carbon network, what is in agreement with the changes of optical constant $n$ and refractive index $k$ obtained from ellipsometry.

\subsection{Thin film deposition in dusty plasmas}

\subsubsection{Film growth}

During plasma processing, the polymerization process does not occur only in the plasma volume leading to dust particle formation, but simultaneously a polymerization takes place on the surface leading to thin film deposition. As it was mentioned before, the presence of dust particles influences the plasma and the sheath properties. Therefore it is expected that the presence of dust particles in the plasma volume during the deposition process will also influence the process of film growth on the surface. Fig. 5.10 presents the growth speed measured during the dust particle formation. The empty wafer is placed on the top and in the middle of the lower electrode and the film growth is measured by the ellipsometer in-situ. The presence of the dust particles inside the plasma is monitored by simultaneous measuring the intensity of the scattered laser light on the half of the distance between the electrodes, $4 \mathrm{~cm}$ far from the wafer in vertical direction.

In Fig. 5.10 it can be seen that the film growth speed follows the periodical behavior of the particle formation process out-of-phase. When the particles are formed in the plasma the growth speed has a low value. A few seconds after the particles are pushed out of the discharge, the film starts growing. Around the middle of the next particle formation cycle the next step in the film growth is finished. The growth precursor particles are consumed from the plasma when the dust particles form and a drastically reduced number of growth precursor particles is left for the film growth so the film growth exhibits a reduction during the particle formation period.

The situation in the thin film growth drastically changes when the molecular hydrogen discharge is ignited and hydrogen atoms are injected into the acetylene discharge. As it was presented in the previous section, after injection of hydrogen atoms, the further particle formation is completely suppressed. Hydrogen atoms cause at the same time an increase of the film growth speed by almost a factor of two, from about $15 \mathrm{~nm} / \mathrm{min}$ to about $30 \mathrm{~nm} / \mathrm{min}$. This situation is presented in Fig. 5.11. 


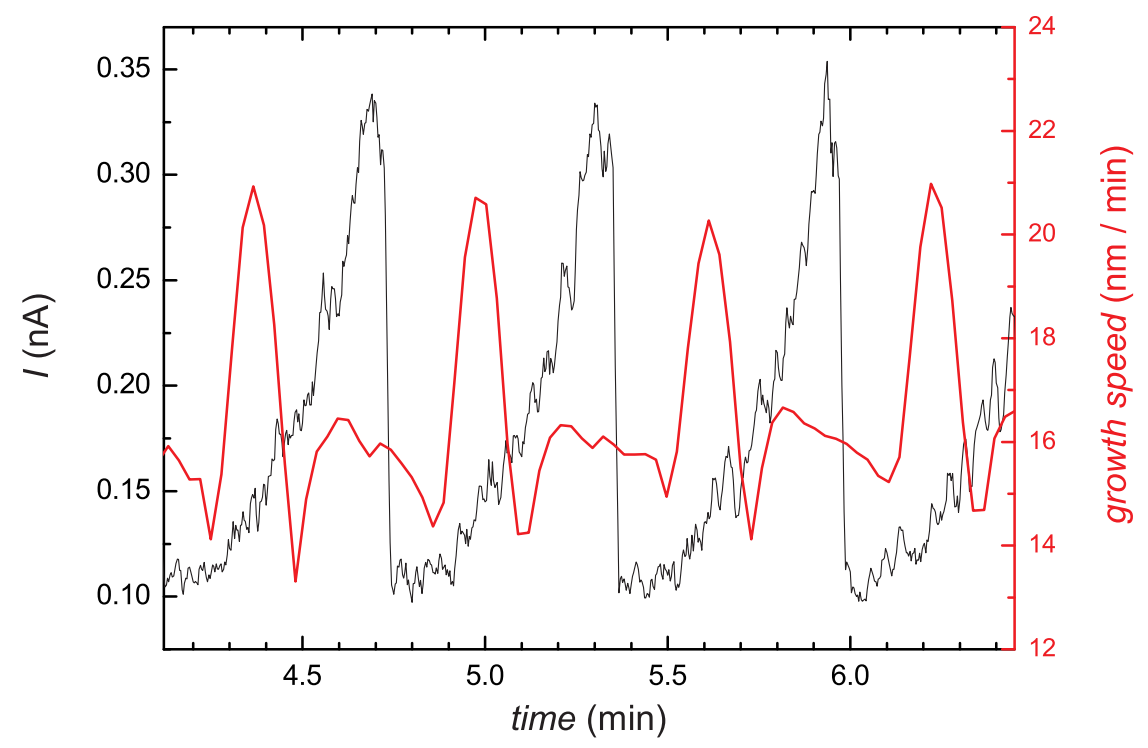

Figure 5.10: Time evolution and periodicity of the thin film growth speed observed by in-situ spectroscopic ellipsometry and of the scattered laser light intensity of the He-Ne laser observed from the acetylene discharge due to the periodic dust formation.

As was already explained in the previous section, the created negative ions are trapped by the positive plasma potential in the plasma bulk and therefore they cannot contribute to the film growth but only to the dust particle formation. On the other hand, positive ions created due to the ionization of the precursor molecules undergo a polymerization further in the plasma volume with $\mathrm{C}_{2} \mathrm{H}_{2}$ molecules leading to the production of higher unsaturated polyacetylene hydrocarbon cations. They can reach the surface and with the sticking coefficient close to unity they are directly incorporated contributing significantly to the film growth.

Neutrals play a role in the film growth, too. For many of them the sticking coefficient is still not known. The ethynyl radical $\left(\mathrm{C}_{2} \mathrm{H}\right)$ is recognized as the dominant neutral growth precursor in acetylene discharge since it has a very high sticking coefficient of about 0.9 [108]. During the polymerization process in the plasma volume, large unsaturated $\mathrm{C}_{2 n} \mathrm{H}_{2}$ molecules are produced in the polymerization reaction of $\mathrm{C}_{2} \mathrm{H}$ with $\mathrm{C}_{2} \mathrm{H}_{2}$ molecules. In reaction with the electrons the $\mathrm{C}-\mathrm{H}$ bond can be broken and corresponding radicals are created. Some possible pathways of creating the radicals in the plasma volume were presented in the previous section. The sticking coefficient for hydrocarbon radicals $\mathrm{C}_{2} \mathrm{H}_{3}, \mathrm{C}_{4} \mathrm{H}_{3}$, and $\mathrm{C}_{6} \mathrm{H}_{3}$ is about 0.35 as it is assumed to be for $s p^{2}$ hybridized radicals [108, 109]. Also different kind of 


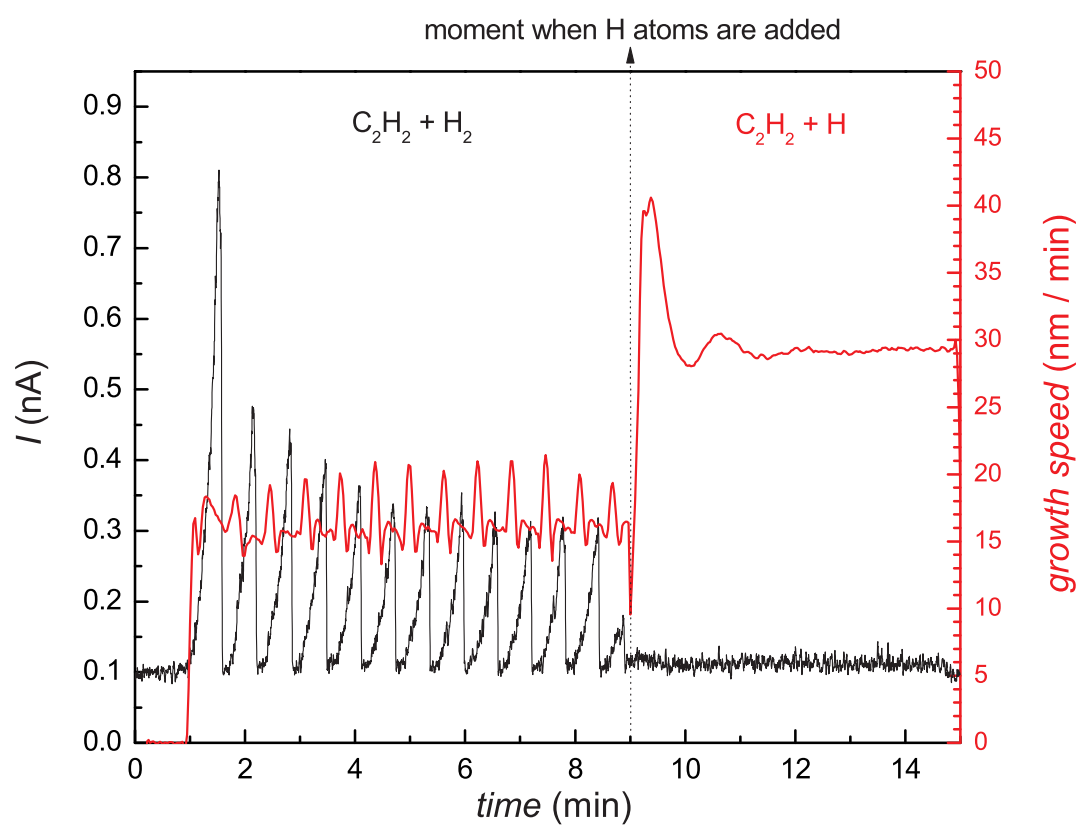

Figure 5.11: The influence of the hydrogen atoms injected from the second discharge into the acetylene plasma on the film growth speed. The hydrogen plasma is ignited at nine minutes after the acetylene plasma is ignited. The vertical dotted line indicates the moment when the second discharge is switched on.

$s p^{3}$ hydrocarbon radicals are formed in the acetylene plasma but they have a very low sticking coefficient of $10^{-3}$ [108], so their contribution to the film growth is minor. On the other hand all these radicals can react with hydrogen atoms creating molecules which return to the plasma.

There is a permanent competition between dust particles and the surface of growing film for the growth precursors. When dust particles form in the plasma volume a reduced amount of precursor particles are left for the film growth. This situation corresponds to a maximum in the intensity of the scattered laser light (due to the particles presence) and a minimum in the growth speed (due to the reduced amount of precursor particles). When the particles are pushed out of the discharge (minimum in the intensity of the scattered laser light) precursor particles can reach the surface of the growing film in higher concentrations (maximum in the growth speed). The time behavior of the $\mathrm{C}_{4} \mathrm{H}_{2}$ biacetylene radical concentration presented in Fig. 5.12 supports this explanation. When hydrogen molecules are injected into the acetylene discharge the concentration of $\mathrm{C}_{4} \mathrm{H}_{2}$ radical has a maximum when the film growth exhibits a minimum. After the injection of hydrogen atoms into the 


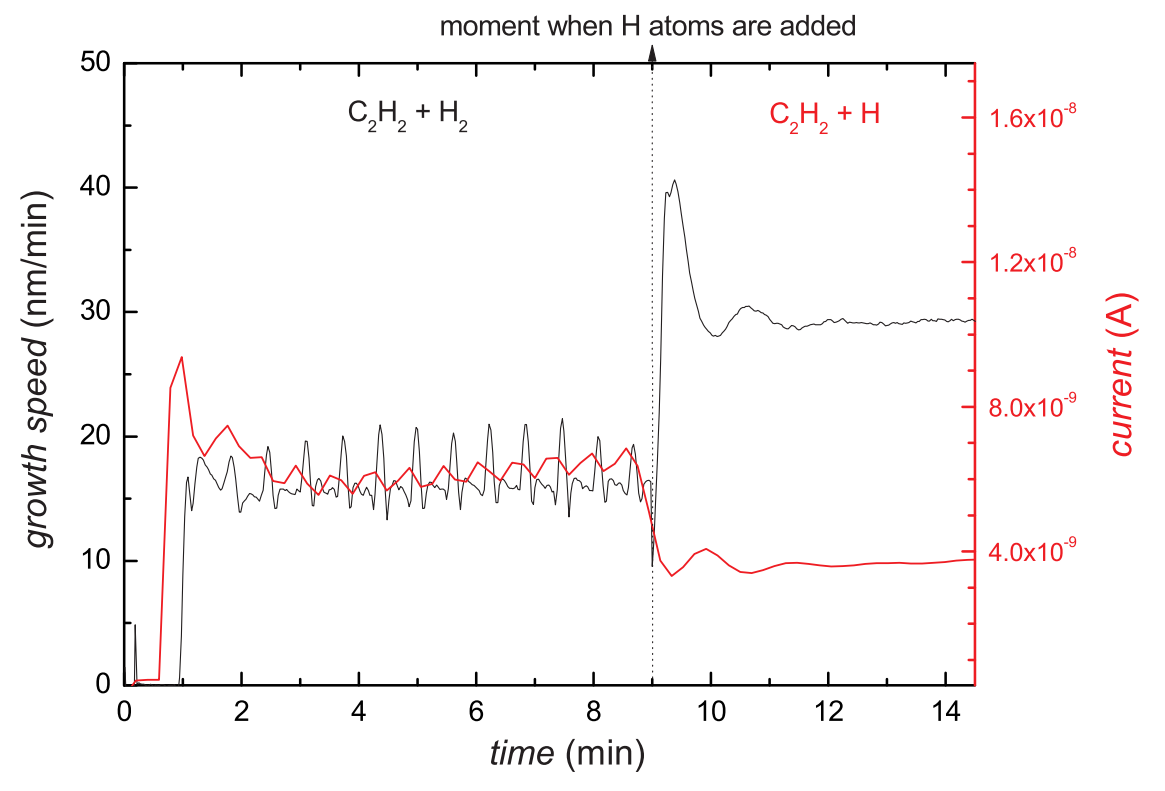

Figure 5.12: Simultaneous temporal behavior of the film growth speed and the $\mathrm{C}_{4} \mathrm{H}_{2}$ mass concentration. The conditions are the same as in Fig. 5.11.

acetylene plasma, the concentration of $\mathrm{C}_{4} \mathrm{H}_{2}$ radicals drops down while the growth speed increases.

Theoretically the atomic hydrogen could enhance the sticking probability of radicals and this induces increased growth rates. But an increase of the sticking coefficient can be attained only for the species with very low sticking coefficient on a nonactivated surface as, for example, $\mathrm{CH}_{3}$ radicals [110]. But $\mathrm{CH}_{\mathrm{x}}$ radicals contribute in the acetylene discharge only with $\sim 0.1 \%$ to the total flux [108], therefore their contribution in the total flux can be neglected. Thus, the activation of the surface by hydrogen atoms will not significantly enhance the growth process by sticking of $\mathrm{CH}_{\mathrm{x}}$ radicals.

On the other hand, the $\mathrm{C}_{2} \mathrm{H}$ radical, which is considered as the growth precursor in the acetylene discharge, has already a very high sticking coefficient of $\sim 0.9 \%$ [108], so that the activation of the growth surface by incoming hydrogen atoms for this kind of radicals does not practically play any role. The increased growth speed after injection of additional flux of hydrogen atoms can thus be assigned to further suppression of particle formation. 

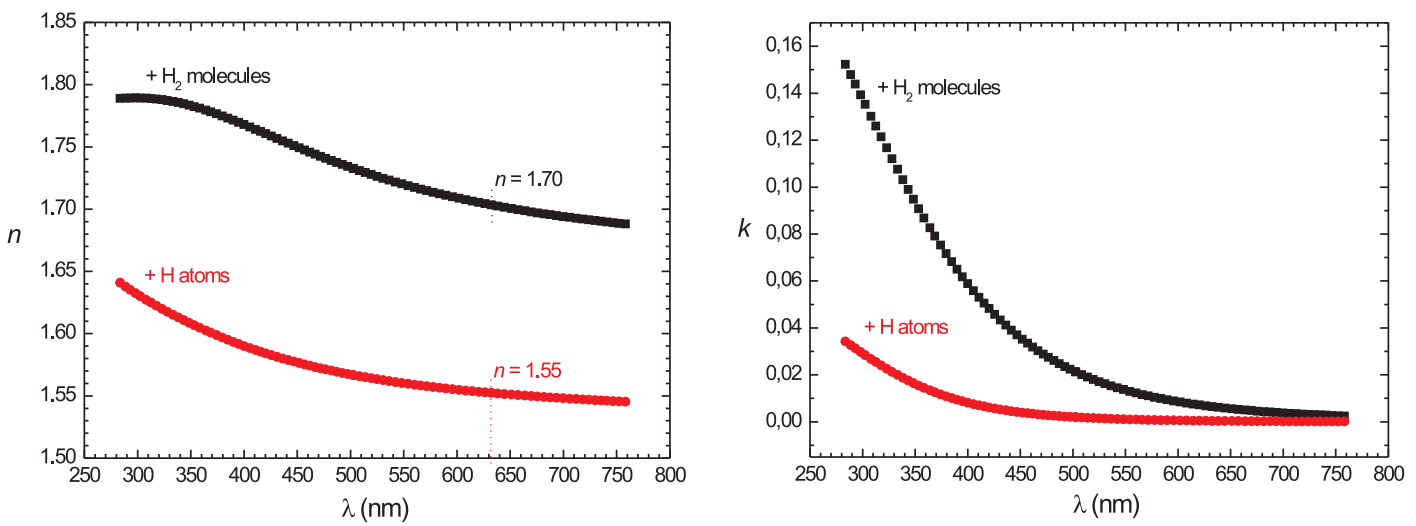

Figure 5.13: Change of the spectroscopic ellipsometry data caused by influence of the hydrogen atoms. On the left graph: the real part of the complex optical constant, refractive index $n$; on the right graph: the imaginary part of the complex optical constant, extinction coefficient $k$. The indicated values of the refractive index are for $\lambda=632.2 \mathrm{~nm}$ wavelength.

\subsubsection{Film properties and film structure}

Fig. 5.13 presents the changes of the complex refractive index (refractive index on the left graph and extinction coefficient on the right graph) of the deposited films under the influence of additional flux of hydrogen atoms. The value of the refractive index $n$ (at $\lambda=632.2 \mathrm{~nm}$ wavelength) is reduced from $n=1.70$ (black curve) to $n=1.55$ (red curve). As it was demonstrated in previous chapter, the lowering of $n$ points out the hydrogenation of the carbon matrix by the additionally injected hydrogen atoms, this becoming softer and polymer-like, richer in $s p^{3} \mathrm{C}-\mathrm{H}$ bonds. At the same time, the lowering of $k$ indicates the reduction of $s p^{2} \mathrm{C}=\mathrm{C}$ bonds through the transformation of $s p^{2} \mathrm{C}=\mathrm{C}$ into $s p^{3}$ hybridized carbon groups.

The above observations on the changes of the optical constants are consistent with the observed changes in the infrared spectra indicating a changed film structure. Fig. 5.14 shows the FTIR spectra measured in the range $2800-3100 \mathrm{~cm}^{-1}$ and the band decomposition. The strong absorption peaks at around $2880 \mathrm{~cm}^{-1}, 2930 \mathrm{~cm}^{-1}, 2970$ $\mathrm{cm}^{-1}, 2990 \mathrm{~cm}^{-1}$, and $3055 \mathrm{~cm}^{-1}$ are dominant in the spectra. This peaks have been assigned to stretching vibrations of $s p^{3} \mathrm{CH}_{3}$ and $s p^{3} \mathrm{CH}_{2}$ symmetric and asymmetric and $s p^{2} \mathrm{C}-\mathrm{H}$ aromatic. The reduction of the peak intensity belonging to the $s p^{2}$ $\mathrm{C}-\mathrm{H}$ aromatic stretching bend indicates the destruction of $\mathrm{C}=\mathrm{C}$ bonds and their saturation with hydrogen atoms. Even though the production of benzene $\left(\mathrm{C}_{6} \mathrm{H}_{6}\right)$ in the plasma is increased after the injection of hydrogen atoms from the second source (presented on the lower graph in Fig. 4.11) the concentration of benzene 

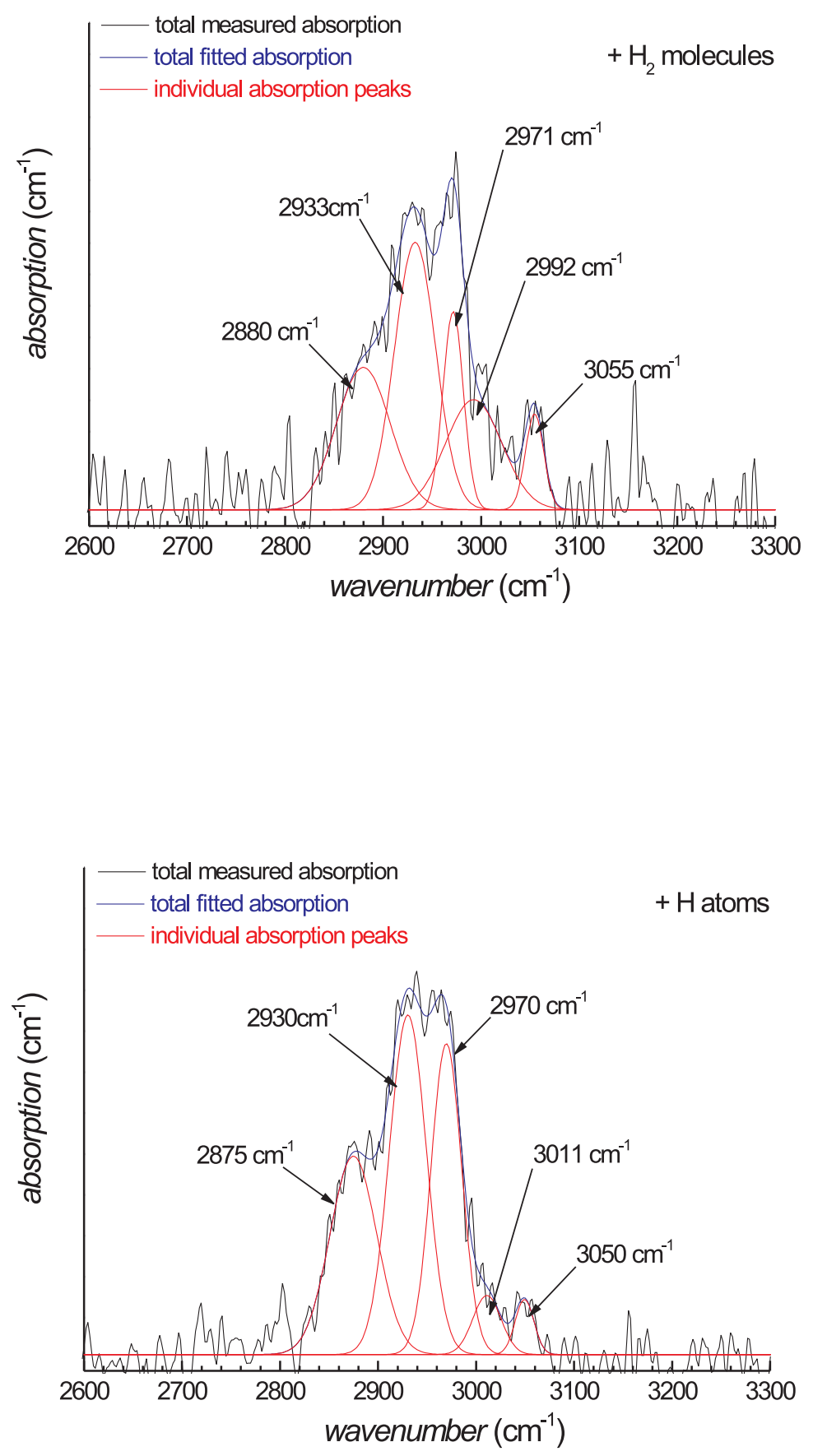

Figure 5.14: Deconvolution of the infrared absorption spectrum of the hydrocarbon films associated with the $\mathrm{C}-\mathrm{H}$ stretch absorption bands. Upper graph: the film deposited with the addition of the hydrogen molecules; lower graph: the film deposited with the addition of the hydrogen atoms. Before the deconvolution both spectra are baseline corrected and their intensities are normalized to unity. The films are of similar thicknesses. 
rings incorporated in the film network is lowered by breaking the $\mathrm{C}=\mathrm{C}$ bonds and the incorporation of hydrogen atoms leads to the formation of dangling bonds.

\subsubsection{Conclusions}

This section contains the results from two groups of investigations. One is about the influence of hydrogen atoms on the deposition and etching speed of amorphous hydrogenated carbon films. Another concerns the influence of hydrogen atoms on the structure of the film. For these investigations a double plasma experimental setup is employed. But this time the double plasma experimental setup consists of an expanding microwave plasma for the creation of active species and the deposition/etching of films while the hydrogen source is the same as in the previous section.

The thickness of the films deposited and etched in this experiment are analyzed ex-situ by means of the ellipsometry and the structure is analyzed by Raman and infrared spectroscopy.

These experiments reveal that the additional flux of hydrogen atoms influences both, the etching and the deposition processes: the deposition rate decreases and the etching rate increases by one order of magnitude in the presence of an additional flux of hydrogen atoms impinging onto the surface. This demonstrates uniquely a pronounced synergistic effect of atomic hydrogen and energetic surface bombardment under real plasma conditions. It is similar to the synergistic effect described by Schwarz-Selinger et al [95], Jacob [96], and Vietzke [102]. The kind of precursor gas and the ion energy also influence the deposition/etching speed.

Ex-situ Raman and infrared spectroscopic measurements show that the additional flux of hydrogen atoms leads to an increase of the hydrogen content in the film by changing the $s p^{2} / s p^{3}$ ratio.

Additionally the thin film deposition in dusty plasmas and the influence of the volume polymerization on the surface polymerization process is investigated in the ICP/UHF setup by means of in-situ ellipsometry. Comparing the temporal evolution of those two processes it can be observed that the growth of the deposited film follows on the same way periodical dust particle formation. As soon as the hydrogen discharge is ignited and hydrogen atoms are added into the acetylene discharge, further particle formation is suppressed and the growth speed of the film is enhanced by almost a factor of 2 . 


\section{Chapter 6}

\section{Summary and Conclusions}

Plasma deposited amorphous hydrogenated carbon films (a-C:H) still attract a lot of interest due to their extraordinary properties. Depending on the deposition conditions (applied power, gas pressure, gas flow rate and bias voltage, temperature, and the gas composition) the films can vary from hydrogenated soft polymer-like via hard diamond-like carbon to pure carbon based tetrahedral amorphous and diamond modifications. The properties of a-C:H films are mainly determined by the bonding hybridization ratio of the carbon atoms in the different hybridization states, i.e. $s p^{3}: s p^{2}: s p^{1}$, and by the hydrogen content.

Carbonaceous plasma grown nanoparticles attract a similarly high attention as a-C:H films do. During the fabrication of integrated circuits from reactive plasmas nanoparticles may be spontaneously created inside the plasma reactor, causing often damage of the devices. On the other hand, in fabrication of nanocrystalline materials, incorporation of nanoparticles into the film matrix improves the films properties.

During the plasma processing hydrogen molecules and atoms are created inherently in all hydrocarbon discharges through the dissociation of precursor molecules. Hydrogen atoms acting together with ions during the film growth can activate or deactivate the surface processes through the creation of dangling bonds or their saturation, leading to an enhancement (synergistic effect) or a reduction (anti-synergistic effect) of the growth speed. Hydrogen atoms also influence the particle formation. These effects are observed and studied in this work.

In order to achieve a better understanding of the role hydrogen atoms play in the formation of carbon based nanoparticles and on the growth/etching speed of the amorphous hydrogenated carbon films experiments were performed with a specially designed double plasma setup. It consists of two combined plasmas. One plasma is used as a source of active species created in the dissociation processes of the precursor gas molecules for the particle formation or for the film deposition. In the case of nanoparticle formation it is an inductively coupled plasma and in the 
case of the investigation of film deposition/etching speed it is a microwave expanding plasma. As a precursor gases are used methane and acetylene. The spatial afterglow of a fully recombined surface wave plasma (second plasma) is used as an independent and controllable source of additional flux of hydrogen atoms. Here the working gas is a flow of molecular hydrogen.

To observe the time evolution of the dust particle formation, in-situ monitoring of the intensity of scattered laser light coming from the discharge is measured while the size and topography of dust particles are investigated ex-situ by means of the secondary electron microscope. The chemical composition of the plasma during the process is monitored by rest gas analyzer (neutrals) and plasma process monitor (positive ions) mass spectrometers.

The measurements of the intensity of scattered laser light show that particles in acetylene discharge spontaneously start forming soon after the discharge is ignited and that particles periodically grow and disappear from the discharge. The particles grown in this inductively coupled discharge have a very broad size distribution and a very smooth surface. The particle growth speed is very high and a few particle generations exist at the same time in the discharge. All this strongly contrasts the situation in capacitively coupled discharge where particles have the growth speed of $\mathrm{nm} / \mathrm{min}$ order, particles are almost monodisperse and have cauliflower shape. During this phase of the experiment the second discharge is switch off, thus only the hydrogen molecules are injected into the acetylene plasma.

The situation drastically changes after the ignition of plasma in molecular hydrogen and the injection of additional flux of hydrogen atoms in the acetylene plasma. Any further particle formation is completely suppressed as long as the hydrogen discharge is on, i.e. the additional amount of hydrogen atoms is injected in the acetylene discharge. After the switching off of molecular hydrogen discharge, i.e. again injecting only hydrogen molecules to the acetylene discharge, the formation of particles starts again spontaneously. A key to the understanding of this effect is the plasma chemistry. From previous work we know that $\mathrm{C}_{2} \mathrm{H}$ radicals are the precursors for driving the polymerization reactions. These radicals, created in an electron induced dissociation reaction:

$$
\mathrm{C}_{2} \mathrm{H}_{2}+\mathrm{e}^{-} \rightarrow \mathrm{C}_{2}+\mathrm{H}+\mathrm{e}^{-},
$$

are destroyed in reaction with hydrogen atoms:

$$
\mathrm{C}_{2} \mathrm{H}+\mathrm{H} \rightarrow \mathrm{C}_{2} \mathrm{H}_{2},
$$

which likely appears to be the most important due to the relatively large cross 
section of this reaction and very high concentration of $\mathrm{C}_{2} \mathrm{H}$ radicals. Acting on this way, hydrogen atoms suppress the particle formation in the very early phase of their genesis - the "chemical phase".

This is confirmed by measuring the mass spectra of neutrals and positive ions. The behavior of the neutral mass spectra in the acetylene discharge with the addition of hydrogen molecules correlates with the periodicity of particle formation.

The injection of additional flux of hydrogen atoms influences the chemical composition of the plasma. More precisely, two groups of masses can be differentiate. In one group are masses whose intensity decreases after the additional injection of hydrogen atoms, as it is the case for the masses $m=50$ (biacetylene) and $m=$ 74 (triacetylene). In the second group are the masses whose intensity decreases, as mass $m=78$ (benzene, $\mathrm{C}_{6} \mathrm{H}_{6}$ ), for example.

The positive ion mass spectra expresses a similar behavior. Most of the masses experience a decrease after the injection of additional amount of hydrogen atoms. However, in the case of positive ions the explanation is not straightforward due to at least two reasons. One reason is that the plasma process monitor measures the flux and not the concentration of positive ions. The second one is that the presence of particles itself influences the plasma parameters and the sheath properties, while the additional flux of hydrogen atoms influences both of them, thus it is not possible to make a distinction between these two influences when they act at the same time. To eliminate the influence of particles on the plasma parameters and the sheath properties, the same experiment is performed under the same experimental conditions but with a reduced flow of acetylene when the particle formation is not observed. The same tendency in formation of higher mass hydrocarbon positive ions exists - hydrogen atoms suppress the polymerization process of higher hydrocarbon positive ions.

The second part of this work is dedicated to investigations of the influence of hydrogen atoms, impinging onto the surface, on the deposition and etching speeds of hydrogenated carbon films and on the influence of hydrogen atoms on the film properties and the structure under plasma conditions. For this investigations a rearranged double plasma setup is employed. The atomic hydrogen source is the same as in the previous experiment but for the creation of active species for deposition/etching process this time an expanding microwave plasma is employed. As precursor gases methane and acetylene are used.

Presented data show that hydrogen atoms strongly influence processes on the surface on the way that the deposition speed decreases and the etching speed increases for one order of magnitude in the presence of an additional flux of hydrogen atoms. 
This is in agreement to the literature, where similar anti-synergistic and synergistic effects, respectively, are observed in experiments with well defined beams of ions, radicals and hydrogen atoms. Here, we demonstrated that under the plasma conditions.

The decrease of the deposition speed arises due to passivation of the created dangling bounds at the surface by impinging hydrogen atoms. While the hydrogenation during the film deposition in methane, maximally saturated (hydrogenated) hydrocarbon, occurs due to hydrogenation of the broken $\mathrm{C}-\mathrm{C}$ bonds by impinging argon ions, that also has as a consequence a shortening of the carbon chains, hydrogenation by incoming hydrogen atoms during the film deposition in acetylene, maximally unsaturated hydrocarbon, mainly occurs via hydrogenation of unsaturated $s p^{2}$ carbon groups increasing the amount of $s p^{3}$ hybridized carbon atoms mainly bonded in the polymeric $\left(\mathrm{CH}_{2}\right)$ groups. Hydrogenation results also in the softening of the film.

In the case of the enhancement of the etching speed, during the simultaneous action of $\mathrm{Ar}^{+}$ions and additional $\mathrm{H}$ atom flux, the synergism is explained by the process of chemical sputtering. Incoming $\mathrm{H}$ atoms passivate dangling bonds not only on the surface but also within the ion penetration range of the energetic $\mathrm{Ar}^{+}$ions, creating $\mathrm{C}_{\mathrm{x}} \mathrm{H}_{\mathrm{y}}$ species. This species then diffuse to the surface and desorb. Since this process occurs not only at the surface but also within the whole ion penetration range it is more efficient then when the passivation of dangling bonds occurs only at the surface.

Incoming hydrogen atoms are incorporated also in the bulk of the film during the growth process and influence its structure. One consequence in the increase of the hydrogen content, reducing the film density and the change in the film structure.

The Raman spectra of films deposited in the mixture of methane with addition of molecular and atomic hydrogen show that incorporated hydrogen atoms reduce the size of the $s p^{2}$ phase which can exist in ring or chain form and slightly influence the trans-polyacetylene phase in the film. On the other hand Raman spectra of the films deposited in acetylene discharges with addition of molecular and atomic hydrogen revealed almost complete destruction of the $s p^{2}$ graphitic sites via the saturation of $\mathrm{C}=\mathrm{C}$ bonds by incoming hydrogen atoms toward the increase of the $s p^{3}$ phase $\left(-\mathrm{CH}_{\mathrm{x}}\right.$ groups) and the amount of bonded hydrogen atoms. This is supported by the infrared spectra revealing additional incorporation of hydrogen atoms in presence of the hydrogen atoms flux. This conclusion is also in good agreement with the decrease of the refractive index $n$ and the extinction coefficient $k$ indicating higher hydrogenation of the film caused by the incorporation of hydrogen atoms in the carbon network during the growth of the film what is usually taken as an indication 
for softening of the film.

This work is additionally extended to investigations of the growth process of amorphous hydrogenated film in the presence of particles. During the deposition process in the mixture of acetylene and molecular hydrogen polymerization occurs in the plasma volume (formation of particles) and on the surfaces (growth of the film). From the comparison of the temporal evolution of these two processes it can be observed that the growth of the deposited film is out-of-phase with the periodicity of the particle formation. This is due to the permanent competition between the particles and the surface of the growing film for the growth precursors.

This work contains the results of the fist time done investigations of the role of hydrogen atoms in the very early stage of the volume polymerization process of hydrocarbon nanoparticle formation. More precisely, the attention is payed to chemistry of the polymerization process and to studding of the suppression of particle formation after the injection of additional flux of hydrogen atoms by reconverting the precursor radicals $\mathrm{C}_{2} \mathrm{H}$ into acetylene molecules. Not only the influence of hydrogen atoms on the volume polymerization process but also the influence of hydrogen atoms on the surface polymerization, i.e. the film growth under the plasma conditions is studied. 


\section{Bibliography}

[1] S. Aisenberg and R. Chabot. J. Appl. Phys., 42(7):2953-2958, 1971.

[2] L. Holland and S. M. Ojha. Thin Solid Films, 38:17, 1976.

[3] F. W. Smith. Mat. Sci. Forum, 52/53:323, 1989.

[4] W. Jacob and W. Möller. Appl. Phys. Lett., 63:1771, 1993.

[5] I. Langmir, C. G. Found, and A. F. Dittmer. Science, 60:392, 1924.

[6] M. Moisan, C. Beaudry, and P. Leprince. Phys. Lett., 50A:125, 1974.

[7] M. Moisan, C. Beaudry, and P. Leprince. IEEE, PS-3:55, 1975.

[8] M. Moisan, C. Beaudry, E. Bloyet, J. M. Gagné, P. Leprince, J. Marec, G. Michel, A. Ricard, and Z. Zakzewski. IEE Conf. Publication, (134):382, 1976.

[9] U. Kortshagen. Habilitationschrift. Plenum Press, 1994.

[10] J. Berndt, D. Douai, and J. Winter. Phys. Plasmas, 9(3):1046-1049, 2002.

[11] K. Behringer. Plasma Phys. Contr. Fusion, 33:997, 1991.

[12] D. Douai. Study of the $N$ yield in a microwave plasma for the deposition of CNH materials: Measurement in the remote plasma, Kinetic study and Etching of a-C:H films by $N$ atoms. PhD thesis, Ruhr-University Bochum, Germany, 2001.

[13] M. Lieberman and A. Lichtenberg. Principles of Plasma Discharges and Material Processing. John Wiley \& Sons, Inc., plasma technology edition, 1994.

[14] Y. P. Raizer. Gas discharge physics. Springer-Verlag Berlin, 1991.

[15] Ch. Scharwitz. Elektronendichtemessung mit einer Plasmaabsorptionssonde. Ruhr-University Bochum, Faculty for Physics and Astronomy, 2004.

[16] A. G. Engelhardt and A. V. Phelps. Phys. Rev. E, 131:2115, 1963. 
[17] G. Baravian, Y. Chouan, A. Ricard, and G. Sultan. J. Appl. Phys., 61:5249, 1987.

[18] Guide to Using WVASE32, Reference Manual. J. A. Woollam Co. Inc.

[19] H. G. Tompkins and W. A. McGahan. Spectroscopic ellipsometry and reflectomerty. John Wiley and Sons, Inc., 1999.

[20] N. B. Colthup, L. H. Daly, and S. E. Wiberley. Introduction to infrared and Raman spectroscopy, page 159. Academic Press, Inc., 1975.

[21] T. Heitz, B. Drévillon, C. Godet, and J. E. Bourée. Phys. Rev. E, 58(20):13 $957-13973,1998$.

[22] J. Ristein, R. T. Stief, L. Ley, and W. Beyer. J. Appl. Phys., 84:3836, 1998.

[23] B. Dischler, A. Bubenzer, and P. Koidl. Solid State Commun., 48(2):105 108, 1983.

[24] A. C. Ferrari and J. Robertson. Phys. Rev. B, 61(20):14 095 - 14 107, 2000.

[25] W. Jacob and M. Unger. Appl. Phys. Lett., 68(4):475-477, 1996.

[26] A. Grill and V. Patel. Appl. Phys. Lett., 60(17):2089-2091, 1992.

[27] A. C. Ferrari and J. Robertson. Physical Review B, 64:075414, 2001.

[28] A. C. Ferrari and J. Robertson. Physical Review B, 63:121405(R), 2001.

[29] J. Jolly and J. Pérrin. Le VIDE n 286, 1997.

[30] S. Selwyn, J. Singh, and R. S. Bennett. J. Vac. Sci. Technol., 4:2758, 1989.

[31] G. S. Selwyn, J. S. Killop, K. L. Haller, and J. J. Wu. J. Vac. Sci. Technol. A, 8:6, 1990 .

[32] D. Samsonova and J. Goree. Phys. Rev. E, 59:1047, 1999.

[33] Y. Watanabe, M. Shiratani, Y. Kubo, I. Ogawa, and S. Ogi. Appl. Phys. Lett., 53:1263, 1988.

[34] M. D. Tanenbaum, A. L. Laracuente, and A. Gallagher. Appl. Phys. Lett., 68:1075-1077, 1996.

[35] Z. Shen, T. Kim, U. Kortshagen, P.H. Mcmurry, and S. A. Campbell. J. Appl. Phys., 94(4):2277-2283, 2003. 
[36] M. Schulze, A. von Keudell, and P. Awakowicz. Plasma Sources Sci. Technol., 15:556-563, 2006.

[37] L. Boufendi, A. Plain, J. P. Blondeau, A. Bouchoule, C. Laure, and M. Toogood. Appl. Phys. Lett., 60:169, 1992.

[38] C. Courteille, C. Hollenstein, J. Dorier, P. Gay, W Scharzenbach, A. A. Howling, E. Bertran, G. Viera, R. Martins, and A. Macarico. J. Appl. Phys., 80:2069, 1996.

[39] C. Cui and J. Goree. IEEE Trans. Plasma Sci., 22:151, 1994.

[40] S. A. Sandford, L. J. Allamandola, A. G. G. M. Tielens, K. Sellgren, M. Tapia, and Y. Pendleton. ApJ, 371:607, 1991.

[41] Y. J. Pendleton, S. A. Sanford, L. J. Allamandola, A. G. G. M. Tielens, and K. Sellgren. ApJ, 437:683, 1994.

[42] D. C. B. Whittet et al. ApJ, 490:729, 1997.

[43] A. Gauger, H.-P. Gail, and E. Sedlmayr. A\&A, 235:345, 1990.

[44] T. Neckel and H. J. Staude. ApJ, 320:L145, 1987.

[45] R. D. Gehrz, J. W. Truran, R. E. Williams, and S. Starrfiled. PASP, 110:3, 1998.

[46] H. W. W. Spoon, A. F. M. Moorwood, K. M. Pontoppidan, J. Cami, D. Lutz M. Kregel, and A. G. G. M. Tielens. A\&A, 402:499, 2003.

[47] R. E. Mason, G. Wright, Y. Pendleton, and A. Adamson. ApJ, 613:770, 2004.

[48] E. Kovacevic, I. Stefanovic, J. Berndt, Y. J. Pendleton, and J. Winter. ApJ, 623:242-251, 2005.

[49] W. W. Stoffels, E. Stoffels, and K. Tachibana. J. Vac. Sci. Technol., 16(1):8795, 1998.

[50] S. M. Collins, D. A. Brown, J. F. O'Hanlon, and R. N. Carlile. J. Vac. Sci. Technol. A, 14(2):634-638, 1996.

[51] A. Bouchoule, A. Plain, L. Boufendi, J. Ph. Blondeau, and C. Laure. J. Appl. Phys., 70(4):1991-2000, 1991. 
[52] Y. Watanabe, M. Shiratani, and K. Koga. Plasma Sources Sci. Technol., 11:A229-A233, 2002.

[53] Ch. Hollenstein. Plasma Phys. Control. Fusion, 42:R93-R104, 2000.

[54] R. J. Seeböck, W. Böhme, W. E. Köhler, M. Römheld, and S. Veprek. Plasma Source Sci. Technol., 3:359-368, 1994.

[55] L. Boufendi, A. Bouchoule, and T. Hbid. J. Vac. Sci. Technol., 14(2):572-576, 1996.

[56] S. Stoykov, C. Eggs, and U. Kotshagen. J. Phys. D: Appl. Phys., 34:21602173, 2001.

[57] Ch. Deschenaux, A. Affolter, D. Magni, Ch. Hollenstein, and P. Fayet. J. Phys. D: Appl. Phys., 32:1876-1886, 1999.

[58] A. Garscadden, B. N. Ganguly, P.D. Haaland, and J. Williams. Plasma Sources Sci Technol., 3:239-245, 1994.

[59] C.-K. Yeon and K.-W. Whang. J. Vac. Sci. Technol. A, 13:2044-2050, 1995.

[60] Ch. Hollenstein, W. Schwarzenbach, A. A. Howling, C. Courteille, J.-L. Dorier, and L. Sansonnens. J. Vac. Sci. Technol. A, 14:535-539, 1996.

[61] H. Kobayashi, A. T. Bell, and M. Shen. Macromolecules, 7:277, 1974.

[62] J. M. Tibbitt, R. Jensen, A. T. Bell, and M. Shen. Macromolecules, 10:647, 1977.

[63] P. Pecher and W. Jacob. Appl. Phys. Lett., 73:31-33, 1998.

[64] W. Möller, W. Fukarek, K. Lange, A. von Keudell, and W. Jacob. Jpn. J. Appl. Phys., 34:2163-2171, 1995.

[65] Ch. Hollensten. Plasma Phys. Control. Fusion, 42:R93 - R104, 2000.

[66] L. Boufendi and A. Bouchoule. Plasma Sources Sci. Technol., 3:262, 1994.

[67] J. Perrin, P. Molinas-Mata, and P. Belenguer. J. Phys. D: Appl. Phys., 27:2499, 1994.

[68] J.-Ch. Schauer. Elektrische Untesuchungen an Argon- und Staub bildenden Plasmen in einer kapazitiv gekoppelten Radiofrequenz-Entladung. RuhrUniversity Bochum, Faculty for Physics and Astronomy, 2003. 
[69] I. Stefanovic, E. Kovacevic, J. Berndt, and J. Winter. New Jour. of Phys., 5:39.1-39.12, 2003.

[70] A. Bouchoule. Technological impact of dusty plasmas. Plenum Press, dusty plasmas edition, 1999.

[71] P. K. Shukla and A. A. Mamun. Introduction to Dusty Plasma Physics. Institute of Physics Publishing Bristol UK, 2002.

[72] M. Lampe, G. Joyce, and G. Ganguli. IEEE Trans. Plasma Sci, 33(1):57-69, 2005.

[73] M. S. Barns, J. H. Keller, J. C. Forser, J. A. O’Neill, and D. K. Coultas. Phys. Rev. Lett., 68:313, 1992.

[74] J. E. Daugherty, R. K. Porteous, and D. B. Graves. J. Appl. Phys., 73:1617$1620,1993$.

[75] S. Hamaguchi and R. T. Farouki. Phys. Rev. E, 49:4430, 1994.

[76] V. E. Fortov, A. V. Ivlev, S. A. Khrapak, A. G. Khrapak, and G. E. Morfill. Physics Reports, 421:1-103, 2005.

[77] S. A. Khrapak and G. E. Morfill. Phys. Rev. E, 69:066411, 2004.

[78] D. B. Graves, J. E. Daugherty, M. D. Kilgore, and R. K. Porteous. Plasma Sources Sci. Technol., 3:433, 1994.

[79] L. Talbot, R. K. Cheng, R. W. Schefer, and D. R. Willis. J. Fluid. Mech., 101:737-758, 1980.

[80] C. Godde. Analyse von Staubpartikeln in einem reaktiven Niedertemperaturplasma. Ruhr-University Bochum, Faculty for Physics and Astronomy, 2005.

[81] E. Kovacevic. Plasma polymerized carbonaceous nanoparticles: application as astroanalog. PhD thesis, Ruhr-University Bochum, Germany, 2006.

[82] S.-H. Hong. From Thin Films to Nanoparticles: Investigation of Polymerization Processes in Capacitively Coupled Hydrocarbon Plasmas. PhD thesis, Ruhr-University Bochum, Germany, 2004.

[83] H. T. Do, G. Thieme, M. Fröhlich, H. Kersten, and R. Hippler. Contrib. Plasma Phys., 45(5-6):378 - 384, 2005. 
[84] S. Hong, J. Berndt, and J. Winter. Plasma Sources Sci. Technol., 12:46 - 52, 2003.

[85] J. Goree, G. E. Morfill, V. N. Tsytovich, and S. V. Vladimirov. Phys. Rev. E, 59(6):7055 - 7067, 1999.

[86] R. K. Janev and D. Reiter. Phys. Plasmas, 11:780, 2004.

[87] K. De Bleeker, A. Bogaerts, and W. Goedheer. Phys. Rev. E, 73:026405, 2006.

[88] S. Hong, J. Berndt, and J. Winter. J. Phys. D, 12:46, 2003.

[89] L. J. Allamandola, A. G. G. M. Tielens, and J. R. Barker. ApJS, 71:733-775, 1989.

[90] C. Barckholtz, T. P. Snow, and V. M. Bierbaum. ApJ, 547:L171 - L174, 2001.

[91] H. Tsai and D. B. Bogy. J. Vac. Sci. Technol., 6(4):2307-2315, 1988.

[92] A. Grill. Diamond and Related Materials, 12:166-170, 2003.

[93] J. Vac. Sci. Technol., 20(3):338-340, 1982.

[94] A. von Keudell, T. Schwarz-Selinger, and W. Jacob. J. Appl. Phys., 76:676678, 2001.

[95] T. Schwarz-Selinger, M. Meier, C. Hopf, A. von Keudell, and W. Jacob. Vacuum, 71:361-376, 2003.

[96] W. Jacob. Thin Solid Films, 326:1, 1998.

[97] J. Roth, E. Vietzke, and A. Haasz. Suppl. Nucl. Fusion, 1:63, 1991.

[98] J. Roth. J. Nucl. Mater., 266:51, 1999.

[99] J. Küppers. Surf. Sci. Rep., 22:249, 1995.

[100] C. Hopf, A. von Keudell, and W. Jacob. Nucl. Fusion, 42:L27-L30, 2002.

[101] A. von Keudell, T. Schwarz-Selinger, and W. Jacob. J. Appl. Phys., 89:29792986, 2001.

[102] E. Vietzke, K. Flaskamp, and V. Phillips. J. Nucl. Mater., 111:763, 1982.

[103] A. von Keudell and W. Jacob. J. Appl. Phys., 79:1092, 1996.

[104] C. Hopf, A. von Keudell, and W. Jacob. J. Appl. Phys., 94:2373-2380, 2003. 
[105] A. von Keudell. Thin Solid Films, 402:1-37, 2002.

[106] H. Biederman and Y. Osada. Plasma Polymerization Processes, volume 3. Elsevier, Amsterdam, palasma technology edition, 1992.

[107] A. Grill. Thin Solid Films, 355-356:189-193, 1999.

[108] C. Hopf, K. Letourneur, W. Jacob, T. Schwarz-Selinger, and A. von Keudell. Appl. Phys. Lett., 74:3800 - 3802, 1999.

[109] A. von Keudell, C. Hopf, T. Schwarz-Selinger, and W. Jacob. Nucl. Fusion, 39:1451, 1999.

[110] C. Hopf, W. Jacob, and A. von Keudell. J. Appl. Phys., 97:094904, 2005. 


\section{CURRICULUM VITAE}

DATE OF BIRTH: 31.07.1966 in Niš, Serbia

CITIZENSHIP: Serbian

EDUCATION:

1981-1985 Gymnasium: Science \& Engineering School "Svetozar Marković", Niš, Group for Biology

1985-1996 Department of Physics, Faculty of Philosophy, University of Niš, Bachelour of Physics

1996-2001 Master studies in Physics of Ionized Gases, Department of Physics, Faculty of Sciences and Mathematics, University of Niš

since 2001 Doctor studies, Institute for Experimental Physics II, Faculty for

Physics and Astronomy, Ruhr-University Bochum, Germany

WORK PLACE:

1996-1998 Professional Collaborator at Department of Physics, Faculty of Sciences and Mathematics, Niš

2000-2001 Professor in the technical secondary school "Mija Stanimirović", Niš 


\section{Acknowledgment}

With the great pleasure I express the deep gratitude to Prof. Dr. Jörg Winter for the opportunity to make my doctor thesis in the Institute for Experimental Physic II. I am also grateful for his valuable discussions, advices and constant interest in my work.

It was the great pleasure to work and discuss with the people in my group. Domi, thank you very much for our friendship, always patient listening, all the time you gave me, and for reading and correcting my manuscript. I want to thank to David for helping me making the first steps in the laboratory and Marc for never let me down during my work. Janine, Jens, my roommates Raphaela and Carsten, Christian, Suk-Ho, Stephy, Johannes, Eva and Ilija, thank you very much for all discussions, help and support you provided to me. I am also very grateful to Prof. Dr. Achim von Keudell for all discussions we had and all help he gave to me. Also thank to people from his group, Timo, Jan, Martin, Jörn, Angel and Angelo for wonderful time I had with them and all help they offered to me.

I owe the gratitude to all our technicians K. Brinkoff, A. Lang, W. Winterhalder, M. Konkowski, C. Hiltner and K. Fiegler for their work and care about my experiment whole the time of my work. Special thanks goes to Frau M. Ocklenburg for taking care about whole administration.

I would like to thank to Prof. Dr. Zoran Petrović for believing in me and all help during my work.

Finnaly, my gratitude goes to my parents, my brother and his small family, and my friends Aleksandra, Lidija, Ljiljana, Čeda and Dejan. Also to Dragan, the special one in my life. 